\title{
Cell Volume-Activated and Volume-Correlated Anion Channels in Mammalian Cells: Their Biophysical, Molecular, and Pharmacological Properties
}

Yasunobu Okada, Toshiaki Okada, Kaori Sato-Numata, Md. Rafiqul Islam, Yuhko Ando-Akatsuka, Tomohiro Numata, Machiko Kubo, Takahiro Shimizu, Ranohon S. Kurbannazarova, Yoshinori Marunaka, and Ravshan Z. Sabirov

Departments of Physiology and Systems Bioscience (Y.O.) and Molecular Cell Physiology (Y.M.), Kyoto Prefectural University of Medicine, Kyoto, Japan; Division of Cell Signaling, National Institute for Physiological Sciences, Okazaki, Japan (Y.O., T.O., M.R.I., M.K., R.Z.S.); Department of Physiology, School of Medicine, Fukuoka University, Fukuoka, Japan (K.S.-N., T.N.); Department of Cell Physiology, Faculty of Pharmaceutical Sciences, Suzuka University of Medical Science, Suzuka, Japan (Y.A.-A.); Department of Pharmaceutical Physiology, Graduate School of Medicine and Pharmaceutical Sciences, University of Toyama, Toyama, Japan

(T.S.); Laboratory of Molecular Physiology, Institute of Bioorganic Chemistry, Academy of Sciences of Uzbekistan, Tashkent, Uzbekistan (R.S.K., R.Z.S.); and Research Institute for Clinical Physiology, Kyoto Industrial Health Association, Kyoto, Japan (Y.M.)

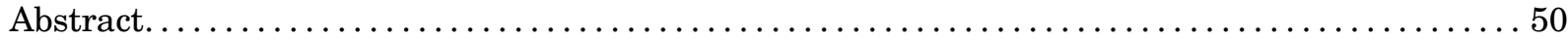

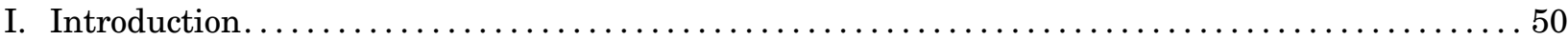

A. Classification and Terminology of Anion Channels.......................... 50

B. Primary Actions of Anion Channel Opening .............................. 51

C. Volume-Controlling Action of Anion Channels ............................... 52

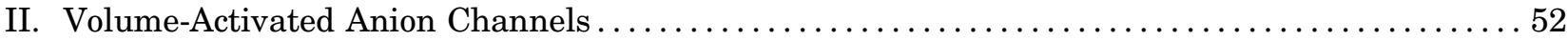

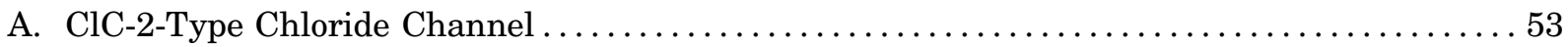

B. Volume-Sensitive Outwardly Rectifying Anion Channel....................... 53

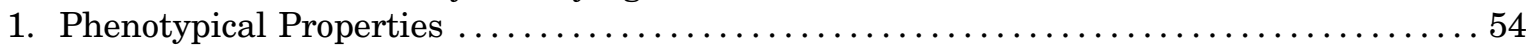

2. Activation Mechanisms ............................................ 54

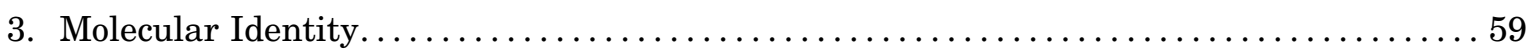

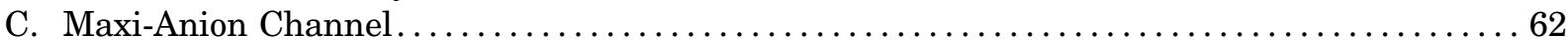

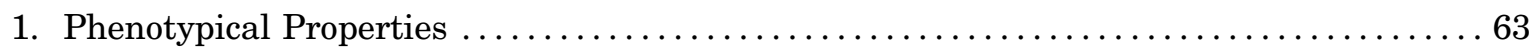

2. Activation Mechanisms .............................................. 64

3. Molecular Identity............................................... 64

III. Volume-Correlated Anion Channels ........................................66 66

A. Cystic Fibrosis Transmembrane Conductance Regulator ..................... 66

B. Calcium-Activated Chloride Channel ................................... 67

C. Acid-Sensitive Outwardly Rectifying Anion Channel ..........................68

IV. Pharmacological Properties of and Distinctions between Volume-Activated Anion Channels

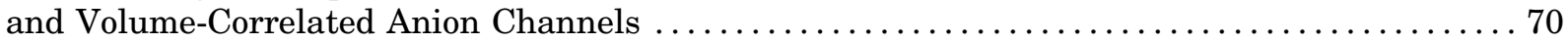

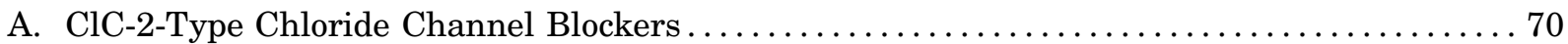

B. Volume-Sensitive Outwardly Rectifying Anion Channel Blockers................... 71

C. Maxi-Anion Channel Blockers........................................ 74

D. Cystic Fibrosis Transmembrane Conductance Regulator Blockers $\ldots \ldots \ldots \ldots \ldots \ldots \ldots \ldots 75$

E. Calcium-Activated Chloride Channel Blockers ............................ 77

F. Acid-Sensitive Outwardly Rectifying Anion Channel Blockers $\ldots \ldots \ldots \ldots \ldots \ldots \ldots \ldots \ldots 78$

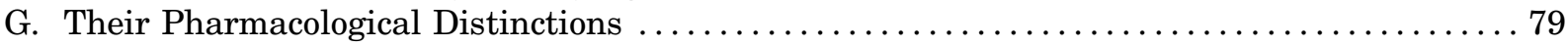

Address correspondence to: Yasunobu Okada, Division of Cell Signaling, National Institute for Physiological Sciences, Higasiyama 5-1, Myodaiji-cho, Okazaki 444-8787, Japan. E-mail: okada@nips.ac.jp

This work was supported by the Japan Society for the Promotion of Science [KAKENHI Grants 26293045 and 17 K19517 (to Y.O.) and 1608510 (to T.O.)].

https://doi.org/10.1124/pr.118.015917. 


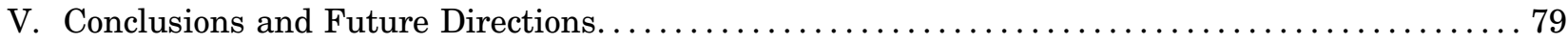

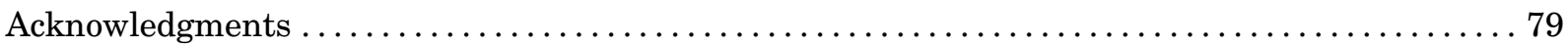

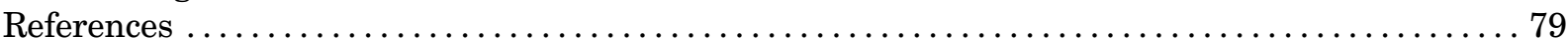

Abstract — There are a number of mammalian anion channel types associated with cell volume changes. These channel types are classified into two groups: volume-activated anion channels (VAACs) and volumecorrelated anion channels (VCACs). VAACs can be directly activated by cell swelling and include the volume-sensitive outwardly rectifying anion channel (VSOR), which is also called the volume-regulated anion channel; the maxi-anion channel (MAC or Maxi-Cl); and the voltage-gated anion channel, chloride channel (ClC)-2. VCACs can be facultatively implicated in, although not directly activated by, cell volume changes and include the cAMP-activated cystic fibrosis transmembrane conductance regulator (CFTR) anion channel, the $\mathrm{Ca}^{2+}$-activated $\mathrm{Cl}^{-}$channel (CaCC), and the acid-sensitive (or acid-stimulated) outwardly rectifying anion channel. This article describes the phenotypical properties and activation mechanisms of both groups of anion channels, including accumulating pieces of information on the basis of recent molecular understanding. To that end, this review also highlights the molecular identities of both anion channel groups; in addition to the molecular identities of ClC-2 and CFTR, those of CaCC, VSOR, and Maxi-Cl were recently identified by applying genome-wide approaches. In the last section of this review, the most up-to-date information on the pharmacological properties of both anion channel groups, especially their half-maximal inhibitory concentrations $\left(\mathrm{IC}_{50}\right.$ values) and voltagedependent blocking, is summarized particularly from the standpoint of pharmacological distinctions among them. Future physiologic and pharmacological studies are definitely warranted for therapeutic targeting of dysfunction of VAACs and VCACs.

\section{Introduction}

Chloride is the most abundant anion in the living human body, constituting approximately $70 \%$ and $65 \%$ of total anionic species of the interstitial fluid and blood plasma, respectively. Although the intracellular solution consists of a large variety of other anionic species including organic anions, inorganic anion $\mathrm{Cl}^{-}$is the most predominant membrane-permeable anion. Therefore, the membrane-spanning channels for anionic electrolytes have often been called chloride channels. A number of chloride channel types have also recently been shown to participate in cell-to-cell signaling by serving as pathways for the release of intracellular organic anions such as glutamate and ATP. Thus, the term anion channel (in lieu of chloride channel) is mainly used throughout this review.

\section{A. Classification and Terminology of Anion Channels}

Mammalian anion channels are ordinarily classified into six major groups according to their activation mechanisms (as listed in Table 1), although some minor types of anion channels may exist depending on the cell type. For example, the outwardly rectifying (depolarizationinduced) $\mathrm{Cl}^{-}$channel in epithelial cells is often associated with unphysiological situations (for review, see Guggino, 1993) and prostaglandin E2 (PGE2)/nitric oxide/cGMPactivated small-conductance anion channels in gastric

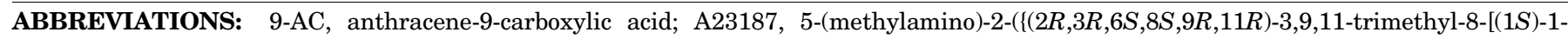
methyl-2-oxo-2-(1H-pyrrol-2-yl)ethyl]-1,7-dioxaspiro[5.5]undec-2-yl\}methyl)-1,3-benzoxazole-4-carboxylic acid; ABC, ATP-binding cassette; ANO, anoctamin; ASOR, acid-sensitive outwardly rectifying anion channel; $[\mathrm{ATP}]_{\mathrm{i}}$, intracellular free ATP concentration; AVD, apoptotic volume decrease; BSP, bromosulfophthalein; $\left[\mathrm{Ca}^{2+}\right]_{i}$, intracellular free $\mathrm{Ca}^{2+}$ concentration; $\mathrm{CaCC}, \mathrm{Ca}^{2+}$-activated $\mathrm{Cl}^{-}$channel; $\mathrm{CaCC}_{\text {inh }}-\mathrm{A} 01$, 6-(1,1-dimethylethyl)-2-[(2-furanylcarbonyl)amino]-4,5,6,7-tetrahydrobenzo[b]thiophene-3-carboxylic acid; CBX, carbenoxolone; CFTR, cystic fibrosis transmembrane conductance regulator; CFTR $_{\text {inh }}-172$, 3-[(3-trifluoromethyl)phenyl]-5-[(4-carboxyphenyl)methylene]-2thioxo-4-thiazolidinone; CHO, Chinese hamster ovary; $\left[\mathrm{Cl}^{-}\right]_{\mathrm{i}}$, intracellular $\mathrm{Cl}^{-}$concentration; DASU-02, N(4-methylphenylsulfonyl)N'-(4-trifluoromethylphenyl)urea; DCPIB, 4-[(2-butyl-6,7-dichloro-2-cyclopentyl-2,3-dihydro-1-oxo-1H-inden-5-yl)oxy]butanoic acid; DIDS, 4,4'-diisothiocyano-2,2' stilbenedisulfonic acid; DIOA, $R$-(+)-[(2- $n$-butyl-6,7-dichloro-2-cyclopentyl-2,3-dihydro-1-oxo-1H-inden5 -yl)oxy] acetic acid; DPC, diphenylamine-2-carboxylate; DUOX, dual oxidase; $E_{\mathrm{Cl}}$, equilibrium potential for $\mathrm{Cl}^{-}$; $\mathrm{ER}$, endoplasmic reticulum; FFA, flufenamic acid; FRT, Fischer rat thyroid; GABA $\mathrm{A}, \mathrm{GABA}_{\mathrm{A}}$ receptor; GAP27, gap junction peptide 27; GlyH-101, $N$-(2naphthalenyl)-((3,5-dibromo-2,4-dihydroxyphenyl)methylene)glycine hydrazide; GlyR, glycine receptor; GSH, glutathione; GTP $\gamma \mathrm{S}$, guanosine 5'-3-O-(thio)triphosphate; H7, 5-(2-methylpiperazin-1-yl)sulfonylisoquinoline dihydrochloride; HEK293, human embryonic kidney 293; HEK293T, human embryonic kidney $293 \mathrm{~T}$; I-V, current-voltage; $\mathrm{KCC}, \mathrm{K}^{+}-\mathrm{Cl}^{-}$cotransporter; LRR, leucine-rich repeat; Maxi-Cl, maxi-anion channel (also MAC); $\left[\mathrm{Mg}^{2+}\right]_{\mathrm{i}}$, intracellular free $\mathrm{Mg}^{2+}$ concentration; NBD, nucleotide binding domain; NFA, niflumic acid; NKCC, $\mathrm{Na}^{+}-\mathrm{K}^{+}-2 \mathrm{Cl}^{-}$cotransporter; NOX, NAD(P)H oxidase; NPPB, 5-nitro-2-(3-phenylpropylamino)benzoic acid; NS3728, $N$-[3,5-bis(trifluoromethyl)-phenyl]- $N$-[4-bromo-2-(1H-tetrazol-5-yl)-phenyl] urea; NVI, necrotic volume increase; PGE2, prostaglandin E2; PGT, prostaglandin transporter; PKA, protein kinase A; PPQ-102, 6,7-dihydro-7,9-dimethyl-6-(5-methyl-2furanyl)-11-phenylpyrimido $\left[4^{\prime}, 5^{\prime}: 3,4\right]$ pyrrolo[1,2-a]quinoxaline-8,10(5H,9)-dione; PTK, protein tyrosine kinase; RD, regulatory domain; ROS, reactive oxygen species; RPE, retinal pigment epithelium; RVD, regulatory volume decrease; siRNA, small interfering RNA; SITS,

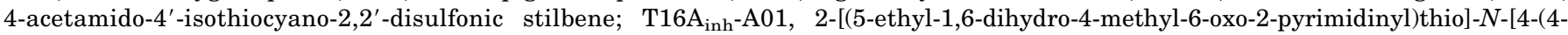
methoxyphenyl)-2-thiazolyl]acetamide; VAAC, volume-activated anion channel; VCAC, volume-correlated anion channel; VRAC, volume-regulated anion channel; VSOAC, volume-sensitive organic osmolyte anion channel; VSOR, volume-sensitive outwardly rectifying anion channel; WW781, 4-[4-[(1E,3E)-5-(1,3-dibutyl-2,4,6-trioxo-1,3-diazinan-5-ylidene)penta-1,3-dienyl]-3-methyl-5-oxo-4H-pyrazol-1-yl]benzenesulfonic acid. 
TABLE 1

Mammalian anion channels: Classification and relation to cell volume and organic anion transport

\begin{tabular}{|c|c|c|c|}
\hline Species & Channel Name & Abbreviation & Molecule \\
\hline \multirow[t]{3}{*}{ Ligand gated } & Glycine receptor & GlyR & GlyR \\
\hline & $\mathrm{GABA}_{\mathrm{A}}$ receptor & $\mathrm{GABA}_{\mathrm{A}} \mathrm{R}$ & $\mathrm{GABA}_{\mathrm{A}}$ \\
\hline & $\mathrm{GABA}_{\mathrm{C}}$ receptor & $\mathrm{GABA}_{\mathrm{C}} \mathrm{R}$ & $\mathrm{GABA}_{\mathrm{C}}$ \\
\hline \multirow{3}{*}{ Voltage gated } & & ClC-2 (one of VAAC and VRAC) & CLCN2 \\
\hline & & ClC-K1 & CLCNK1 \\
\hline & & ClC-K2 & CLCNK2 \\
\hline cAMP activated & $\begin{array}{l}\text { Cystic fibrosis transmembrane } \\
\text { conductance regulator }\end{array}$ & CFTR (one of VCAC and VSOAC) & CFTR \\
\hline \multirow{2}{*}{$\mathrm{Ca}^{2+}$ activated } & & & TMEM16B/ANO2 \\
\hline & & & TMEM16F/ANO6 \\
\hline \multirow[t]{2}{*}{ Volume activated } & $\begin{array}{l}\text { Volume-sensitive outwardly rectifying } \\
\text { anion channel }\end{array}$ & $\begin{array}{l}\text { VSOR (one of VAAC, VRAC, }{ }^{a} \\
\text { and VSOAC) }\end{array}$ & $\mathrm{LRRC} 8 \mathrm{~A}+8 \mathrm{C} / 8 \mathrm{D} / 8 \mathrm{E}\left(+?^{c}\right)$ \\
\hline & Maxi-anion channel & $\begin{array}{l}\text { Maxi-Cl (one of VAAC, VRAC, }{ }^{a} \\
\text { VSOAC, and MAC-1 }{ }^{b} \text { ) }\end{array}$ & $\operatorname{SLCO} 2 \mathrm{~A} 1\left(+?^{c}\right)$ \\
\hline Acid activated & $\begin{array}{l}\text { Acid-sensitive outwardly rectifying } \\
\text { anion channel }\end{array}$ & ASOR (one of VCAC) & $?$ \\
\hline
\end{tabular}

${ }^{a}$ The VRAC abbreviation used in this work (for volume-regulated anion channel) is sometimes also referred to as the volume-regulatory anion channel.

${ }^{b}$ Maxi-Cl is the major type of maxi-anion channel (MAC-1) (see the text for details).

${ }^{c}$ The question mark represents some missing molecular component which may exist to reproduce a full set of phenotypes (see the text for details).

parietal cells (Sakai et al., 1989, 1995). Ligand-gated anion channels are called ionotropic receptors, because they are coupled to the glycine receptor (GlyR), $\mathrm{GABA}_{\mathrm{A}}$ receptor $\left(\mathrm{GABA}_{\mathrm{A}} \mathrm{R}\right)$, or $\mathrm{GABA}_{\mathrm{C}}$ receptor. Voltage-gated anion channels are formed by several types of $\mathrm{ClC}$ family members, including ClC-1, ClC-2, ClC-K1(Ka), and $\mathrm{ClC}-\mathrm{K} 2(\mathrm{~Kb})$. Anion channels activated by intracellular signals cAMP and $\mathrm{Ca}^{2+}$ are called the cAMPactivated cystic fibrosis transmembrane conductance regulator (CFTR) $\mathrm{Cl}^{-}$channel and the $\mathrm{Ca}^{2+}$-activated $\mathrm{Cl}^{-}$channel (CaCC), respectively. Although CFTR activation is regulated by cAMP-induced activation of protein kinase A (PKA), its opening and closure are rather directly gated by binding of intracellular ATP and release of its hydrolytic product, respectively. Thus, CFTR may also be classified as a ligand-gated anion channel (Kirk and Wang, 2011). A distinct type of anion channel is the acid-sensitive outwardly rectifying anion channel (ASOR), which is quickly activated by extracellular acidification and characterized by sharp outward rectification. The remaining two types of anion channels are slowly activated by cell swelling and are referred to as the volume-sensitive anion channels: the volume-sensitive outwardly rectifying anion channel (VSOR) is distinctly characterized by mild outward rectification, whereas the most predominant type of maxi-anion channel (MAC; also called the Maxi-Cl, see section II.C), is distinctly characterized by a large unitary ohmic [current-voltage $(I-V)$ linear] conductance of hundreds of picosiemens.

Among these mammalian anion channels, a number are implicated in cell volume changes. VSOR, Maxi-Cl, and ClC-2 are directly activated by cell swelling (Strange et al., 1996) and are thus collectively dubbed the volume-activated anion channels (VAACs) (Okada et al., 2009a,b) (Table 1). Although CFTR, CaCC, and ASOR cannot be directly activated by cell swelling per se, they can be facultatively implicated in changes in cell volume. Here, these anion channels are collectively called the volume-correlated anion channels (VCACs) (Table 1). Although VSOR alone has often been called the volume-regulated anion channel (VRAC) (Table 1) or $I_{\mathrm{Cl} \text {,swell }}$, this terminology may be misleading because Maxi-Cl and ClC-2 are also literally regulated by volume and activated by cell swelling. In addition, the VRAC abbreviation could also be confusingly assigned to volume-regulatory anion channels (Table 1). VRAC has also sometimes been referred to as the volume-sensitive organic osmolyte anion channel (VSOAC) (Table 1), taking its capability to release intracellular organic anions into consideration (Strange et al., 1996). However, VSOR, Maxi-Cl, and CFTR can serve as pathways for the efflux of organic signaling solutes such as glutamate and glutathione (GSH) (Hudson, 2001; Sabirov et al., 2016); therefore, they could also be called VSOACs (Table 1).

\section{B. Primary Actions of Anion Channel Opening}

The opening of anion channels on the plasma membrane results in the shift of membrane potential to the equilibrium potential for $\mathrm{Cl}^{-}\left(E_{\mathrm{Cl}}\right)$ and the net transport of anions across the cell membrane. The former action is not necessarily coupled to the latter action for $\mathrm{Cl}^{-}$, because no electrochemical driving force is given for the $\mathrm{Cl}^{-}$transport when the membrane potential happens to reach the $E_{\mathrm{Cl}}$ value.

The intracellular $\mathrm{Cl}^{-}$concentration $\left(\left[\mathrm{Cl}^{-}\right]_{\mathrm{i}}\right)$ is normally controlled by the balance of counteracting actions of two types of electroneutral cation-chloride cotransporters (Fig. 1): the $\mathrm{Na}^{+}-\mathrm{K}^{+}-2 \mathrm{Cl}^{-}$cotransporter (NKCC) and the $\mathrm{K}^{+}-\mathrm{Cl}^{-}$cotransporter (KCC). The predominant activities of $\mathrm{KCC}$ and $\mathrm{NKCC}$ maintain the $\left[\mathrm{Cl}^{-}\right]_{\mathrm{i}}$ level below and above the $E_{\mathrm{Cl}}$ value, respectively (Russell, 2000; Blaesse et al., 2009; Payne, 2009). Since the $\left[\mathrm{Cl}^{-}\right]_{\mathrm{i}}$ is lower than the value determined by its 


\section{A KCC dominant}

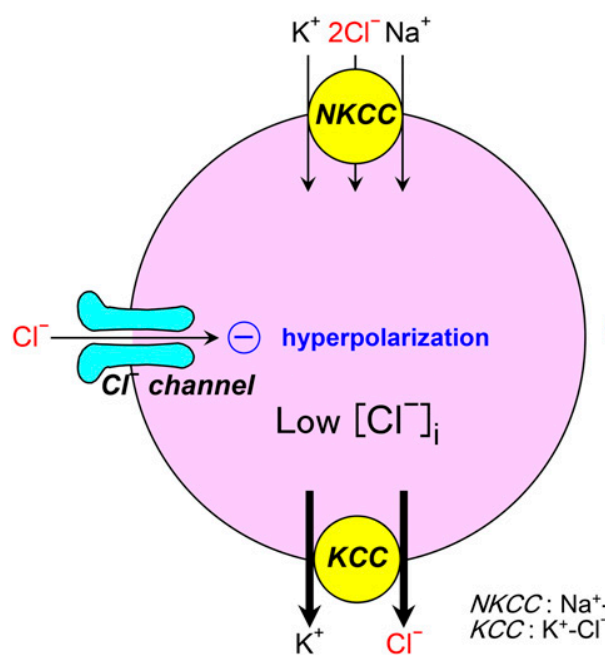

\section{B NKCC dominant}

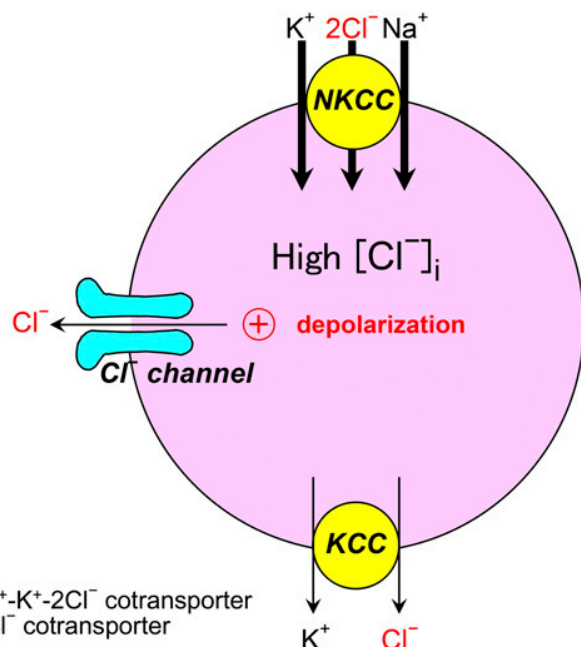

Fig. 1. Primary actions of opening of anion channels in either a KCC-dominant case (A) or an NKCC-dominant case (B) (see the text for details).

electrochemical equilibrium in mature central neurons and skeletal muscle cells, in which KCC is constitutively operating, opening of neural $\mathrm{GABA}_{\mathrm{A}} \mathrm{R}$ and GlyR as well as muscular ClC-1 gives rise to hyperpolarization (Fig. 1A), thereby stabilizing or suppressing their excitability. In contrast, activation of $\mathrm{CaCC}$ and $\mathrm{GABA}_{\mathrm{A}} \mathrm{R} / \mathrm{GlyR}$ brings about depolarization (Fig. 1B) in smooth muscle cells and immature neurons, respectively; this triggers or enhances their excitability, because $\mathrm{Cl}^{-}$is normally above the electrochemical equilibrium in these NKCCdominant cells (Chipperfield and Harper, 2000; Blaesse et al., 2009; Payne, 2009).

In most epithelial cells in which $\mathrm{Cl}^{-}$is maintained at a concentration higher than the equilibrium value by the predominant operation of NKCC (Russell, 2000), opening of anion channels results in efflux of $\mathrm{Cl}^{-}$out of cells (Fig. 1B). Thus, CFTR and CaCC are involved in $\mathrm{Cl}^{-}$secretion or absorption, depending on their location on the apical (luminal) or basolateral (serosal) membrane, respectively, in many epithelial tissues, including the intestinal and airway tracts. In addition, ClC-K2 is involved in $\mathrm{Cl}^{-}$reabsorption in the thick ascending limb of the loop of Henle in the kidney.

\section{Volume-Controlling Action of Anion Channels}

The most important secondary physiologic action of anion channel opening is to regulate or change the cell volume by triggering $\mathrm{KCl}$ flux, because $\mathrm{K}^{+}$conductance exceeds $\mathrm{Cl}^{-}$conductance under resting conditions in most cells. Regulation of cell volume is fundamental to the survival and function of animal cells under both physiologic and pathophysiologic conditions (for reviews, see Lang, 1998; Okada, 1998b, 2004; Hoffmann and Pedersen, 2006; Hoffmann et al., 2009). Among the three types of VAACs, VSOR and Maxi-Cl are involved in cell volume regulation after osmotic cell swelling and thus may be referred to as volume-regulatory anion channels (Table 1). VSOR is the most typical swelling-activated anion channel in most animal cells and plays an effective role in providing a pathway for anion efflux during the regulatory volume decrease (RVD) after cell swelling (for reviews, see Okada and Hazama, 1989; Okada, 1997). Maxi-Cl is also involved in the RVD process directly by providing the volume-regulatory pathway for anion efflux (see Okada et al., 2009b) or indirectly by releasing ATP, which stimulates P2Y receptors in an autocrine fashion (Kurbannazarova et al., 2011; Islam et al., 2012). The cardiac isoform of CFTR may be involved in the RVD process in cardiac myocytes under special conditions in which $\beta$-adrenergic receptors are stimulated (Wang et al., 1997). On the contrary, some other types of anion channels, such as CaCC, ASOR, and CFTR (called VCACs; Table 1), may facultatively or accidentally lead to changes in cell volume. This article focuses on the properties, molecular identities, functions, and pharmacology of these VAACs and VCACs.

\section{Volume-Activated Anion Channels}

VAACs are directly, but not solely, activated by cell swelling. For instance, activation of ClC-2, VSOR, and Maxi-Cl is also brought about by acidic $\mathrm{pH}$, reactive oxygen species (ROS), and membrane patch excision, respectively, even in the absence of a volume increase. All VAACs are not necessarily volume regulatory, because ClC-2 is not involved in the RVD process (Bond et al., 1998), whereas VSOR and Maxi-Cl exert volume-regulatory actions under normal conditions (Okada, 1997; Sabirov and Okada, 2004a). Under apoptotic conditions, however, VSOR activity leads to sustained cell shrinkage, called an apoptotic volume decrease (AVD) (Maeno et al., 2000; Shimizu et al., 
2004), without attaining volume regulation. These VAACs show distinguishable voltage dependence from each other; that is, $\mathrm{ClC}-2$, VSOR, and Maxi-Cl exhibit inward, outward, and no rectification, respectively (Okada et al., 2009b).

\section{A. ClC-2-Type Chloride Channel}

The ClC-2 anion channel was cloned by Jentsch's group (Thiemann et al., 1992) and belongs to the gene family of ClC channel/transporter, which consists of nine different members in mammals (Jentsch et al., 2002). ClC-2 is ubiquitously expressed and activated not only by cell swelling (Gründer et al., 1992) but also by strong membrane hyperpolarization (Thiemann et al., 1992) and by extracellular acidification (Pusch and Jentsch, 1994; Jordt and Jentsch, 1997). The channel is dependent on voltage and is inactive at normal resting potentials that are more positive than $-90 \mathrm{mV}$ (Thiemann et al., 1992); however, ClC-2 becomes activated at physiologic membrane potentials after cell swelling (Gründer et al., 1992). The voltage dependence of this channel is also characterized by inward rectification and inactivation of the tail macroscopic current upon a depolarizing pulse applied after hyperpolarization (Fahlke, 2001). ClC-2 single-channel conductance is small (2 to $3 \mathrm{pS}$ ) (Weinreich and Jentsch, 2001), and its anion selectivity is a type of strong electric field (Eisenman sequence IV) with a permeability sequence of $\mathrm{Cl}^{-}>\mathrm{Br}^{-}>\mathrm{I}^{-}$ (Thiemann et al., 1992; Jordt and Jentsch, 1997). Activation of ClC-2 does not depend on intracellular ATP (Park et al., 1998; Rutledge et al., 2002), which is in contrast to the intracellular ATP dependence of VSOR (for review, see Okada, 1997).

Thus far, ClC-2 is suggested to be involved in a number of physiologic functions, including colonic $\mathrm{Cl}^{-}$ transport (Catalán et al., 2002; Lipecka et al., 2002), gastric acid secretion (Malinowska et al., 1995), $\mathrm{GABA}_{\mathrm{A}} \mathrm{R}$-mediated inhibitory synaptic input (Smith et al., 1995) by controlling the $\left[\mathrm{Cl}^{-}\right]_{\mathrm{i}}$ level in neurons (Staley et al., 1996; Földy et al., 2010), lung development (Murray et al., 1995), and early nephrogenesis (Huber et al., 1998). Nonetheless, ClC-2-deficient mice were found to exhibit neither seizure nor prominent abnormalities in the development of the lung and kidney as well as in epithelial $\mathrm{Cl}^{-}$transport and acid secretion, apart from degeneration of the retina and testis (Bösl et al., 2001).

Since studies showed that ClC-2 was activated by hypotonicity-induced cell swelling in a number of cell types, including Xenopus oocytes transfected with ClC-2 (Gründer et al., 1992; Jordt and Jentsch, 1997; Furukawa et al., 1998), insect Sf9 cells transfected with ClC-2 (Xiong et al., 1999), and native mouse atrial and ventricular myocytes (Duan et al., 2000), it was expected to play a role in cell volume regulation after swelling (RVD). The data suggesting such a volumeregulatory role of ClC-2 were reported in nonmammalian
ClC-2-transfected cells (Furukawa et al., 1998; Xiong et al., 1999) and in native mammalian cells (Roman et al., 2001). However, studies in Xenopus oocytes (Furukawa et al., 1998) and Sf9 cells (Xiong et al., 1999) were conducted in nonmammalian overexpression systems, and no direct evidence for RVD was shown in the former study. Although another study was performed in nontransfected rat hepatoma tissue culture cells (Roman et al., 2001), the conclusions were merely based on indirect observations using anti-ClC-2 antibodies. Moreover, the property of swelling-activated $\mathrm{Cl}^{-}$currents shown by Roman et al. (2001) looks completely distinct from inward-rectifier ClC-2, exhibiting mild outward rectification similar to that of VSOR currents. In addition, the possibility that ClC-2 is involved in the RVD process was ruled out by a study performed in native human colonic T84 cells (Bond et al., 1998) on the basis of findings showing that a specific blocker of ClC-2, $\mathrm{Cd}^{2+}$, failed to affect the RVD and that swelling-induced augmentation of endogenous inwardrectifier ClC-2 currents occurred only after prior activation by hyperpolarizing voltages. Furthermore, the involvement of ClC-2 in RVD was refuted by observations that parotid acinar cells from Clcn2 knockout mice showed RVD with similar efficiency to that for wildtype littermates (Nehrke et al., 2002). Taken together, we conclude that ClC-2 is activated by cell swelling but is not a major contributor to the RVD process.

\section{B. Volume-Sensitive Outwardly Rectifying Anion Channel}

VSOR (also called VRAC) is ubiquitously expressed and directly activated by cell swelling induced by decreased extracellular or increased intracellular osmolality. In 1988, two groups functionally discovered this channel independently in human epithelial cells (Hazama and Okada, 1988) and in human lymphoid cells (Cahalan and Lewis, 1988). The single-channel event with an intermediate unitary conductance (10-90 pS), which could sometimes be observed during recording of the whole-cell current under nonswollen conditions, was suggested to be responsible for the swelling-induced macroscopic current in human epithelial T84 cells, based on similarities in their voltage dependence (Worrell et al., 1989). Using a double patch-clamp technique under swollen conditions, direct evidence for the causal involvement of intermediate single-channel events in macroscopic VSOR currents was provided by simultaneous recordings of the single-channel and whole-cell currents in single human epithelial Intestine 407 cells (Okada et al., 1994b; Petersen et al., 1994) and mouse cortical neurons (Inoue and Okada, 2007; Okada et al., 2009b).

VSOR activity plays a primary role in cell volume regulation after swelling, called RVD, by serving as the volume-regulatory $\mathrm{Cl}^{-}$exit pathway (Hazama and Okada, 1988; Okada and Hazama, 1989; Kubo and 
Okada, 1992). As a natural consequence, VSOR activity is secondarily involved in a variety of physiologic cell functions that are inevitably associated with whole or local cell swelling, such as cell proliferation or mitosis, cell migration, and cell differentiation (for reviews, see Eggermont et al., 2001; Hoffmann et al., 2009; Akita and Okada, 2014; Pedersen et al., 2015, 2016). In addition, VSOR is known to serve as a pathway for swelling-induced efflux of organic signaling solutes such as glutamate (Kimelberg et al., 1990; Strange et al., 1996; Kirk, 1997; Liu et al., 2006, 2009; Okada et al., 2009b; Akita and Okada, 2014; HyzinskiGarcía et al., 2014; Planells-Cases et al., 2015; Lutter et al., 2017; Schober et al., 2017). Furthermore, VSOR is involved in the induction of apoptosis (Maeno et al., 2000; Shimizu et al., 2004), as well as to anticancer drug resistance (Lee et al., 2007; Shimizu et al., 2008; Sørensen et al., 2016) and transport (Planells-Cases et al., 2015).

1. Phenotypical Properties. A large number of studies clarified the phenotypical properties of VSOR (see reviews by Okada et al., 1994a; Strange et al., 1996; Nilius et al., 1997a; Okada, 1997, 2006; Shimizu et al., 2008; Akita and Okada, 2014) as follows: volume sensitivity, mild outward rectification (Fig. 2A), voltageand time-dependent inactivation (Fig. 2, A and B), intermediate single-channel conductance (Fig. 2B), low-field-strength anion selectivity with Eisenman sequence type $\mathrm{I}\left(\mathrm{I}^{-}>\mathrm{Br}^{-}>\mathrm{Cl}^{-}>\mathrm{F}^{-}\right)$, nonhydrolytic requirement of intracellular ATP, sensitivity to intracellular free $\mathrm{Mg}^{2+}$, and open-channel block by extracellular ATP. The mild outward rectification of VSOR is its most characteristic biophysical property, which is distinct from two other VAACs [inward-rectifier ClC-2 and ohmic (I-V linear) Maxi-Cl] and from three other VCACs [ohmic CFTR, sharp outward-rectifier CaCC, and ASOR]. The intermediate unitary conductance of VSOR is distinct from the small unitary conductance of ClC-2 ( 2 to $3 \mathrm{pS})$, CFTR (6-12 pS), CaCC (0.45-1.2 pS), and ASOR (4-7 pS) and from the large unitary conductance of Maxi-Cl (300-500 pS). Recent nonelectrolyte partition studies revealed that the cut-off radius of the narrowest constriction inside the VSOR pore is $0.63 \mathrm{~nm}$ (Ternovsky et al., 2004), which is much smaller than that inside the Maxi-Cl pore (approximately $1.3 \mathrm{~nm}$; Sabirov and Okada, 2004b) and larger than that inside the CFTR pore (approximately $0.27 \mathrm{~nm}$; Linsdell et al., 1997). The low-field-strength halide anion selectivity of VSOR is similar to Maxi-Cl, CaCC, and ASOR but distinct from the higher-field anion selectivity of ClC-2 $\left(\mathrm{Cl}^{-}>\mathrm{Br}^{-}>\mathrm{I}^{-}>\mathrm{F}^{-}\right.$: Eisenman type IV) and CFTR $\left(\mathrm{Br}^{-}>\mathrm{Cl}^{-}>\mathrm{I}^{-}>\mathrm{F}^{-}\right.$: Eisenman type III). The voltage-dependent blocking of outward (not inward) VSOR currents by extracellular ATP at $>0.1 \mathrm{mM}$ is one of the unique characteristics of VSOR (Jackson and Strange, 1995; Tsumura et al., 1996). This is due to plugging of the anion-conducting pathway of VSOR by ATP, because the VSOR pore radius is nearly the same as the effective radius of ATP (approximately $0.6 \mathrm{~nm})$.

The dependence of VSOR on intracellular ATP is its most important physiologic property (Jackson et al., 1994; Oiki et al., 1994), which also distinguishes it from ClC-2 and Maxi-Cl, both of which are rather inhibited by intracellular ATP (Rutledge et al., 2002; Sabirov and Okada, 2009). Under constant intracellular free $\mathrm{Mg}^{2+}$ concentration $\left(\left[\mathrm{Mg}^{2+}\right]_{\mathrm{i}}\right)$ conditions, instantaneous $\mathrm{Cl}^{-}$ currents recorded at $+40 \mathrm{mV}$ in human epithelial Intestine 407 cells increased with the increasing intracellular free ATP concentration $\left([\mathrm{ATP}]_{\mathrm{i}}\right)$, as shown in Fig. 3A. The half-maximal effective concentration $\left(\mathrm{EC}_{50}\right)$ for ATP was very low $(0.014 \mathrm{mM}$ at $1 \mathrm{mM}$ free $\mathrm{Mg}^{2+}$ ). Even when intracellular $\mathrm{Mg}^{2+}$ ions were largely chelated by EDTA $(5 \mathrm{mM})$, VSOR currents were not attenuated in the presence of intracellular ATP (Fig. 3B; at $\left.\left[\mathrm{Mg}^{2+}\right]_{\mathrm{i}}=10^{-5} \mathrm{mM}\right)$. Therefore, it appears that free ATP, but not Mg-bound ATP, activates the channel, and the role of free ATP is not mediated by its hydrolysis but rather presumably by its binding to some functional VSOR component. This result is consistent with previous observations that a nonhydrolyzable ATP analog ( $\beta, \gamma$-imidoadenosine $5^{\prime}$-triphosphate) and a slowly hydrolyzable ATP analog (adenosine 5'-o-13thiotriphosphate) did not abolish but rather could substitute, at least in part, for ATP in human epithelial Intestine 407 cells (Oiki et al., 1994), as observed in other cell types (Díaz et al., 1993; Jackson et al., 1994).

VSOR activity is known to be suppressed by an increase in cytosolic free $\mathrm{Mg}^{2+}$ (Oiki et al., 1994; Okada, 1997). In the presence of a constant $[\mathrm{ATP}]_{i}$ $(0.1 \mathrm{mM})$, the current amplitude was decreased (as shown in Fig. 3B), in a concentration-dependent manner, by increases in $\left[\mathrm{Mg}^{2+}\right]_{i}$ or by concomitant increases in the concentration of the intracellular Mg-ATP complex. If Mg-ATP is an inhibitory factor, the inhibition curve plotted on the $\left[\mathrm{Mg}^{2+}\right]_{\mathrm{i}}$ axis must shift to the left (toward lower $\left[\mathrm{Mg}^{2+}\right]_{\mathrm{i}}$ ) by elevation of $[\mathrm{ATP}]_{\mathrm{i}}$, because the concentration of the intracellular Mg-ATP complex is increased by elevation of $[\mathrm{ATP}]_{\mathrm{i}}$ at fixed $\left[\mathrm{Mg}^{2+}\right]_{\mathrm{i}}$ according to the law of mass action. On the contrary, increased $[\mathrm{ATP}]_{\mathrm{i}}$ brought about a shift of the $\left[\mathrm{Mg}^{2+}\right]_{\mathrm{i}^{-}}$ inhibition curve to the right, in the opposite direction (Fig. 3B). Thus, it appears that the channel activity is suppressed by free $\mathrm{Mg}^{2+}$ ions but not by the ATP-bound form of $\mathrm{Mg}$. The half-maximal inhibitory concentration $\left(\mathrm{IC}_{50}\right)$ for free $\mathrm{Mg}^{2+}$ was $0.32 \mathrm{mM}$ at $0.01 \mathrm{mM}[\mathrm{ATP}]_{\mathrm{i}}$ and $1.9 \mathrm{mM}$ at $0.1 \mathrm{mM}[\mathrm{ATP}]_{\mathrm{i}}$. The normalized peak $I-V$ relationship was little affected by altering $\left[\mathrm{Mg}^{2+}\right]_{\mathrm{i}}$ (Fig. 3C). Thus, intracellular $\mathrm{Mg}^{2+}$ suppresses VSOR currents in a voltage-independent manner.

2. Activation Mechanisms. The mechanism of swelling-induced VSOR activation has long been discussed (for reviews, see Nilius et al., 1997a; Okada, 1997; Hoffmann et al., 2009; Okada et al., 2009a; Akita and Okada, 2014). Although many fluid-soluble signals 
A

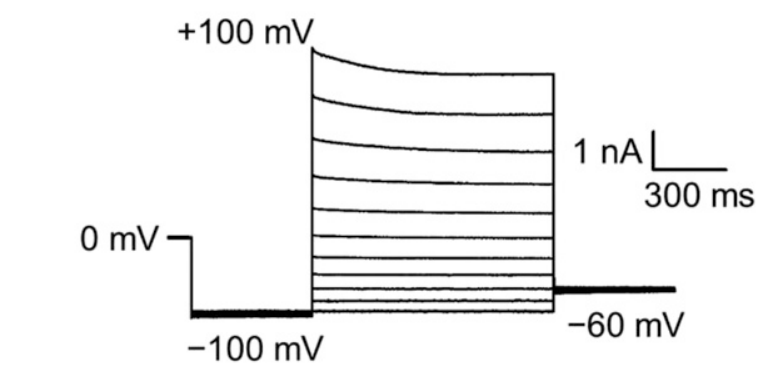

B

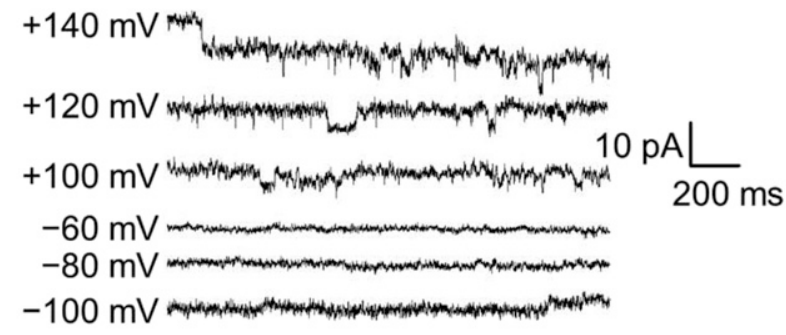

C

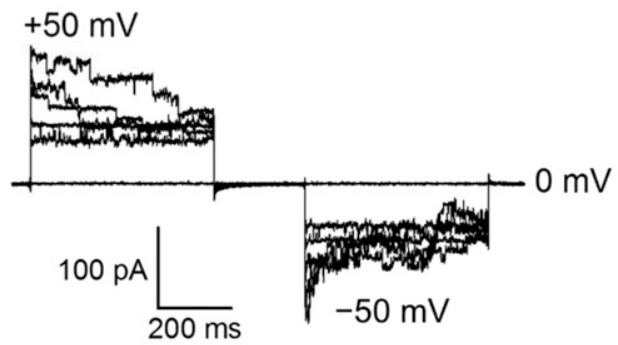

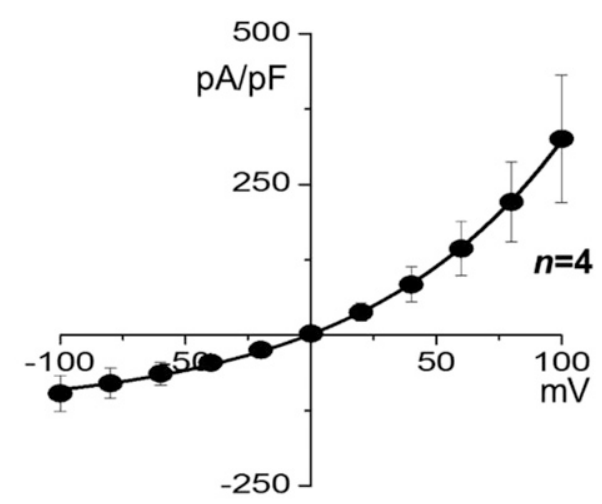
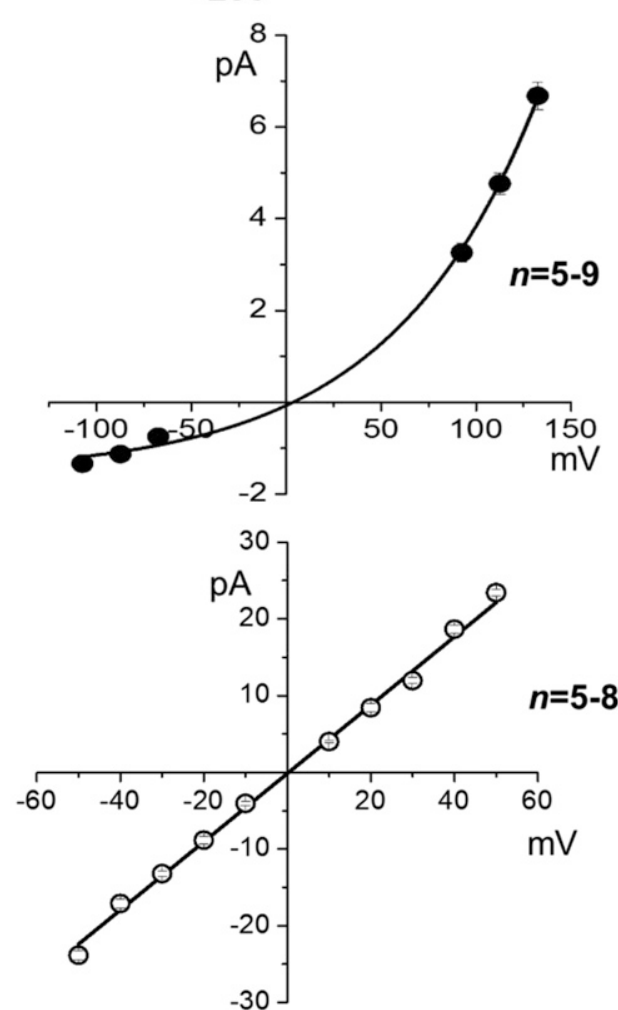

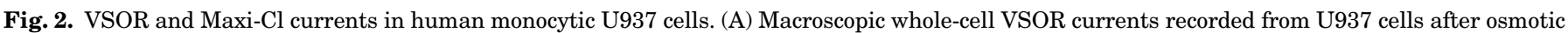

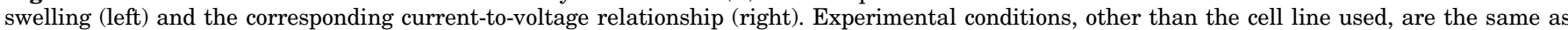

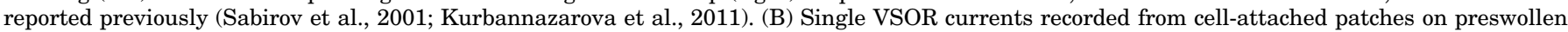

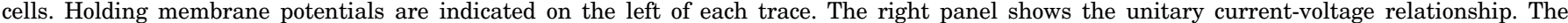

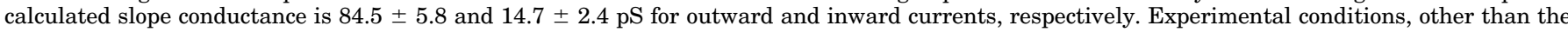

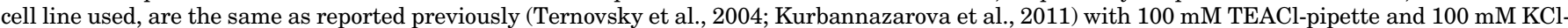

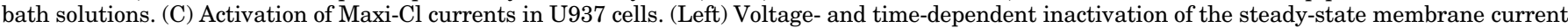

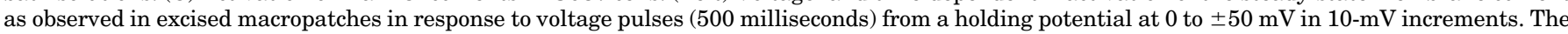

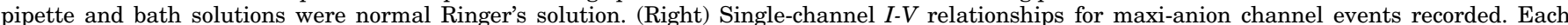

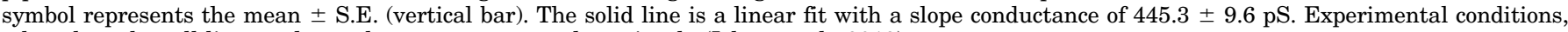
other than the cell line used, are the same as reported previously (Islam et al., 2012).

including a number of kinases and small $\mathrm{G}$ proteins have been suggested to be involved in the mechanism, they have only partial or permissive effects (Okada et al., 2009a).

Direct activation of VSOR by increased membrane stretch or tension can be excluded from the possible mechanism in light of the following observations. First, cell swelling does not necessarily bring about membrane stretch, because the cell membrane has sufficient reserves (namely, infoldings or invaginations) to allow for several-fold volume increases without producing plasmalemmal stretch (Levitan and Garber, 1996; Okada, 1997, 2004; Groulx et al., 2006; Pangrsic et al., 2006). Second, in the cell-attached configuration, membrane stretch induced by applying negative pressure via a patch pipette failed to activate VSOR on nonswollen Intestine 407 cells and did not affect single-channel activity on the swollen cells (Okada, 1997). A lack of association between stretch-activated and swellingactivated $\mathrm{Cl}^{-}$currents was also demonstrated in human hepatocellular carcinoma cells (Mao et al., 2011) and in human neutrophils (Behe et al., 2017). 

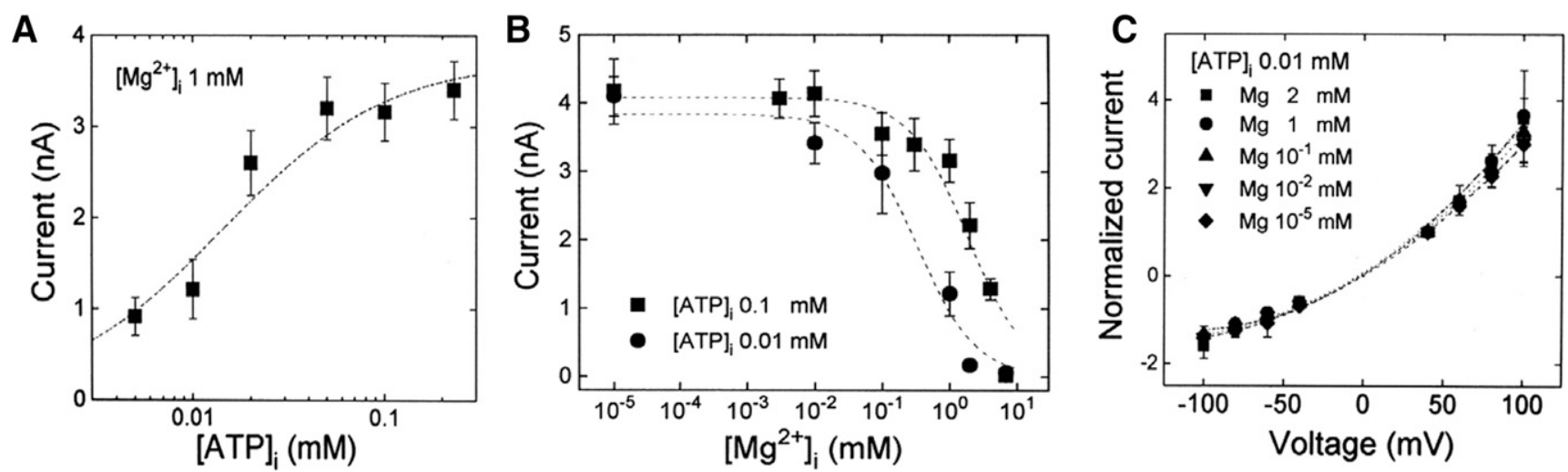

Fig. 3. $\mathrm{Cl}^{-}$current amplitudes plotted against $[\mathrm{ATP}]_{\mathrm{i}},\left[\mathrm{Mg}^{2+}\right]_{\mathrm{i}}$, and voltages. Each symbol represents the mean current of four different Intestine 407 cells. Vertical bars represent the S.E. (A) $[\mathrm{ATP}]_{\mathrm{i}}$ dependence of the peak $\mathrm{Cl}^{-}$currents measured at $+40 \mathrm{mV}$ at a fixed $\left[\mathrm{Mg}^{2+}\right]_{\mathrm{i}}(1 \mathrm{mM})$. Data points were fitted by the following equation: $I=I_{\max }\left([\mathrm{ATP}]_{\mathrm{i}} / \mathrm{EC}_{50}\right) /\left\{1+\left([\mathrm{ATP}]_{\mathrm{i}} / \mathrm{EC}_{50}\right)\right\}$. $(\mathrm{B})\left[\mathrm{Mg}^{2+}\right]_{\mathrm{i}}$ dependence of the peak $\mathrm{Cl}^{-}$currents measured at $+40 \mathrm{mV}$ at a fixed $[\mathrm{ATP}]_{\mathrm{i}}(0.1$ or $0.01 \mathrm{mM})$. Data points were fitted by the following equation: $I=I_{\max } /\left\{1+\left(\left[\mathrm{Mg}^{2+}\right]_{i} / \mathrm{IC}_{50}\right)\right\}$. (C) Current-voltage relationships. Normalized peak $\mathrm{Cl}^{-}$currents $\left(I_{\mathrm{V}} / I_{+40-\mathrm{mV}}\right)$ were measured at different $\left[\mathrm{Mg}^{2+}\right]_{\mathrm{i}}$ and fixed $[\mathrm{ATP}]_{\mathrm{i}} \cdot \mathrm{Mg}^{2+}$ did not change the reversal potential, indicating that the $\mathrm{Mg}^{2+}$ effect is independent of voltage and that $\mathrm{Mg}^{2+}$ cannot permeate the channel. Experimental conditions are the same as reported previously (Oiki et al., 1994).

Instead of membrane tension, unfolding of the membrane invaginations is likely important for channel activation, because the single-channel event of VSOR could be recorded only when the patch pipette was attached to the preswollen cell but not when giga-sealed patches had been formed before the cell was rendered swollen (Okada, 1997, 1998a) and also because the density of whole-cell VSOR currents increased in proportion to the square of the cell diameter (namely, the outer surface area of cell) (Miwa et al., 1997; Okada, 1997; Morishima et al., 2000). Since the swellinginduced activation of VSOR is attained in a manner independent of exocytotic insertion (Okada et al., 1992), VSOR proteins may exist in a largely inactivated state on the plasma membrane prior to a hypotonic challenge and become activated by cell swelling or membrane unfolding. Assuming that the preexisting VSOR protein may readily interact with other proteins (e.g., cytoskeletal or actin-binding proteins) in such a confined space within infoldings filled with the actin-based meshwork, Okada (1997) put forward the "unfolding-induced protein-protein interaction hypothesis" for the VSOR activation mechanism, referred to here as the "type A mechanism" (Fig. 4).

Our more recent work (Ando-Akatsuka et al., 2012) confirmed this hypothesis by the following step-by-step studies in human embryonic kidney $293 \mathrm{~T}$ (HEK293T) cells. First, we searched a cytoskeleton-binding protein, which becomes associated with the cell membrane upon cell swelling; we then found that it is an actinbinding protein, $\alpha$-actinin-4 (ACTN4), which is known to be present ubiquitously in nonmuscle cells (Sjöblom et al., 2008). Second, ABCF2, a cytosolic member of the ATP-binding cassette (ABC) transporter superfamily, was identified as the binding partner of ACTN4 by protein overlay assays combined with proteomics approaches. Third, overexpression and small interfering RNA (siRNA)-mediated downregulation of ABCF2 expression reduced and enhanced VSOR activity, respectively. Thus, we conclude that swelling-induced VSOR activation is attained by membrane unfoldinginduced protein-protein interactions between ACTN4 and $\mathrm{ABCF} 2$, which prevents $\mathrm{ABCF} 2$ from suppressing VSOR activity presumably by releasing ABCF2 from VSOR protein (Fig. 4, type A mechanism). Because the submembrane actin ring is disrupted by cell swelling (Levitan et al., 1995; Morán et al., 1996; Pedersen et al., 1999; Carton et al., 2003; Erickson et al., 2003; Pritchard and Guilak, 2004), ACTN4 might be released from actin, mobilized onto the membrane-rich fraction, and thereby become available for the interaction with ABCF2 (AndoAkatsuka et al., 2012). Since another ABC protein (CFTR) is known to downregulate VSOR activity (Vennekens et al., 1999; Ando-Akatsuka et al., 2002), there is a possibility that VSOR interacts with $\mathrm{ABC}$ proteins other than ABCF2.

Activation of VSOR is provoked even in the absence of cell swelling or membrane unfolding under conditions in which the ROS level is increased (Fig. 4, type B mechanism). Swelling-independent, $\mathrm{H}_{2} \mathrm{O}_{2}$-induced activation of VSOR was originally found independently in 2004 by three groups (Browe and Baumgarten, 2004; Shimizu et al., 2004; Varela et al., 2004) and was confirmed by subsequent studies (Wang et al., 2005; Jiao et al., 2006; Varela et al., 2007; Harrigan et al., 2008; Liu et al., 2009; Deng et al., 2010a,b; Crutzen et al., 2012; Holm et al., 2013; Shen et al., 2014; Xia et al., 2016; Wang et al., 2017a,b). In addition, for the first time, VSOR per se was identified as the anion channel responsible for the $\mathrm{Cl}^{-}$efflux causing apoptotic cell shrinkage, called AVD (Maeno et al., 2000), which was observed after stimulation with an intrinsic mitochondrion-mediated apoptosis inducer (staurosporine) due to increased ROS production via $\mathrm{NAD}(\mathrm{P}) \mathrm{H}$ oxidase (NOX) (Shimizu et al., 2004) (Fig. 4, type B mechanism). Then, ROS-mediated VSOR activation 


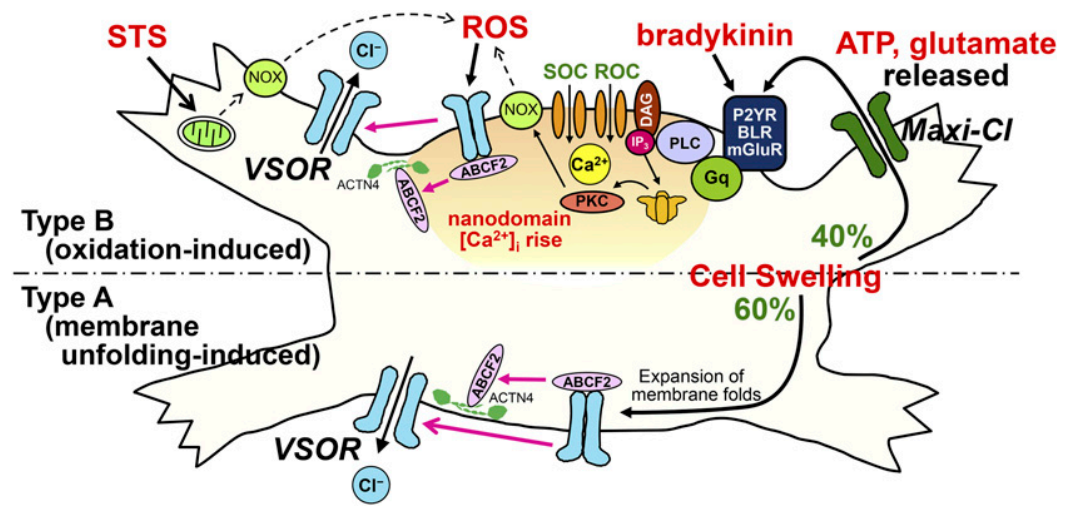

Fig. 4. Dual mechanisms for VSOR activation. The bottom half represents the membrane unfolding-induced (type A) mechanism, which takes place upon cell swelling, and the top half represents the oxidation-induced (type B) mechanism, which takes place upon receptor stimulation (see the text for details and other abbreviations). BKR, bradykinin receptor; DAG, diacylglycerol; Gq, heterotrimeric G protein that activates phospholipase $\mathrm{C}_{\text {; }} \mathrm{IP}_{3}$, inositol trisphosphate; mGluR, metabotropic glutamate receptor; P2YR, P2Y purinergic receptor; PKC, protein kinase C; PLC, phospholipase C; ROC, receptor-operated channel; SOC, store-operated channel.

was observed without cell swelling under stimulation with a variety of apoptosis inducers, including an endoplasmic reticulum (ER) stress-mediated apoptosis inducer (tunicamycin; Shen et al., 2014), a stressinduced apoptosis inducer (ceramide; Raucci et al., 2010), and an anticancer drug (doxorubicin; Kumagai et al., 2012). An extrinsic death receptor-mediated apoptosis inducer, tumor necrosis factor $\alpha$, was also reported to induce ROS-mediated (oxidation-induced) VSOR activation in mouse proximal convoluted tubule cells (l'Hoste et al., 2010) and mouse aortic smooth muscle cells (Matsuda et al., 2010b), although not in human HeLa cells (Shimizu et al., 2004). Baumgarten's group showed that oxidation-induced VSOR activation in cardiac myocytes is brought about by stimulation of angiotensin II receptor type 1 (Browe and Baumgarten, 2004; Ren et al., 2008), the epidermal growth factor receptor (Browe and Baumgarten, 2006), and the endothelin A receptor (Deng et al., 2010a) in rabbit ventricular myocytes. We also showed that ROSmediated VSOR activation in astrocytes under isotonic conditions can be elicited by stimulation of Gq proteincoupled receptors such as the bradykinin $B_{2}$ receptor (Liu et al., 2009; Akita and Okada, 2011), purinergic P2Y receptors (Akita et al., 2011), and metabotropic glutamate receptor 5 (Akita and Okada, 2014). As summarized in Fig. 4 (type B mechanism), we revealed that this receptor-mediated VSOR activation is induced by NOX-mediated ROS production and is controlled via high-concentration regions of intracellular $\mathrm{Ca}^{2+}$ (called " $\mathrm{Ca}^{2+}$ nanodomains") in the immediate vicinity of open $\mathrm{Ca}^{2+}$-permeable channels such as store- and receptoroperated channels (Akita and Okada, 2011; Akita et al., 2011). Such NOX-mediated ROS production controlled by $\mathrm{Ca}^{2+}$ nanodomains may also account for the mechanism of adenosine-induced, purinergic P1 receptormediated activation of VSOR currents in kidney tubular cells, because this was found to be coupled to $\mathrm{Ca}^{2+}$ entry through $\mathrm{Ca}^{2+}$-permeable channels sensitive to $\mathrm{La}^{3+}$ and
$\mathrm{Gd}^{3+}$ (Rubera et al., 2001). In this regard, it is worth noting that leucine-rich repeats (LRRs) containing 8A (LRRC8A), which was recently identified as one of the core components of VSOR (Qiu et al., 2014; Voss et al., 2014), was found to colocalize and interact with NOX1 (Choi et al., 2016).

Since membrane unfolding- and oxidation-induced activation was shown to be additive under certain conditions (Gradogna et al., 2017), type A and type B mechanisms would be independent of each other. When cells respond to hypo-osmotic stress not only with osmotic cell swelling but also with ATP release, VSOR activation can dually be attained by the $\mathrm{Ca}^{2+}$-independent, membrane unfolding-induced type A mechanism and by the $\mathrm{Ca}^{2+}$ nanodomain-dependent, oxidationinduced type B mechanism (Fig. 4). As shown in Fig. 4, in fact, the VSOR current activated upon osmotic swelling was estimated to be composed of approximately $40 \%$ for the oxidation-induced, $\mathrm{Ca}^{2+}$ nanodomainmediated component and another approximately $60 \%$ for the unfolding-induced, $\mathrm{Ca}^{2+}$-independent component in mouse astrocytes (Akita et al., 2011).

There is a possibility that even in the oxidationinduced (type B) mechanism for VSOR activation, ABCF2 is released from the VSOR protein and then binds to ACTN4. In fact, as shown in Fig. 5, exposure to $\mathrm{H}_{2} \mathrm{O}_{2}$ facilitated ACTN4 mobilization onto the radioimmunoprecipitation assay buffer-insoluble membrane- and cytoskeleton-rich $(10,000 \mathrm{~g}$ pellet) fraction (Fig. 5, A and B) and then promoted the ABCF2-ACTN4 interaction, which was monitored by coimmunoprecipitation, in the radioimmunoprecipitation assay buffer-insoluble fraction (Fig. 5, C and D). Thus, it appears that the dissociation of ABCF2 from VSOR is the final common step for dual mechanisms, which include membrane unfolding- and oxidation-induced VSOR activation.

The VSOR current was activated without visible cell swelling by intracellular dialysis with guanosine 
A

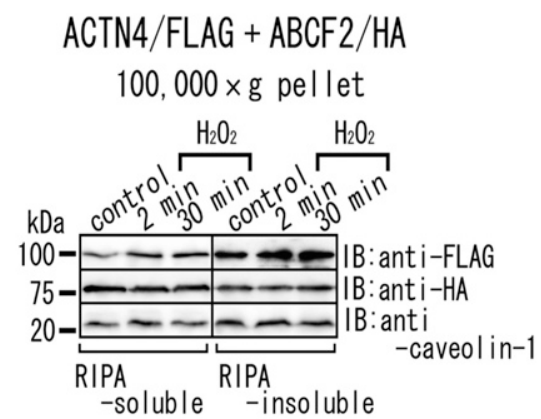

B

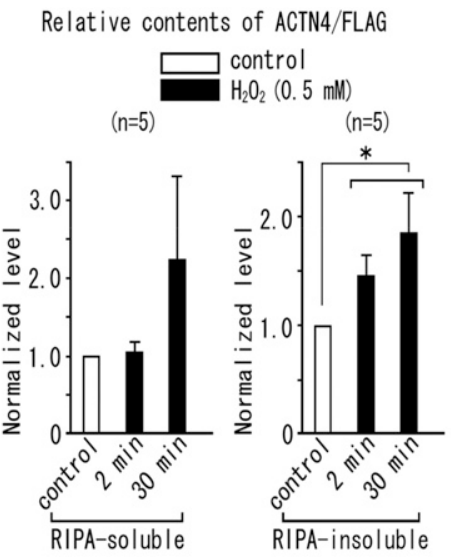

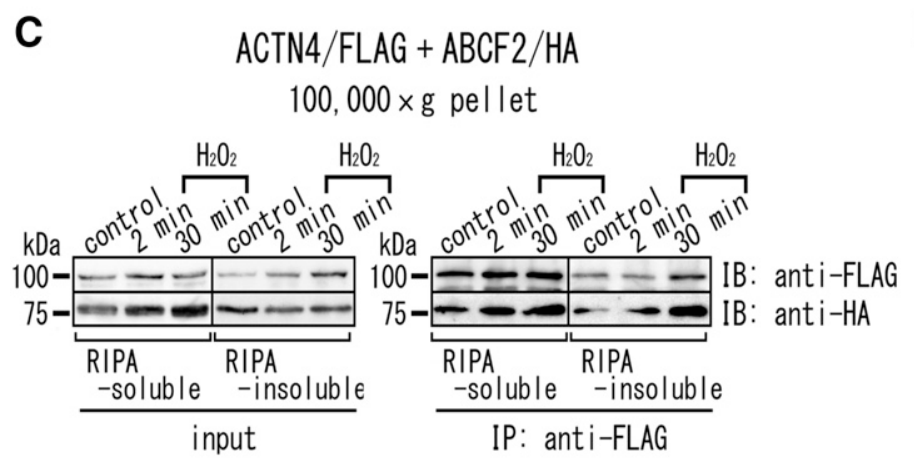

D Relative contents of co-precipitated ABCF2

Fig. 5. Effects of $\mathrm{H}_{2} \mathrm{O}_{2}$ stimulation on the subcellular localization of ACTN4 and molecular interaction between ACTN4 and ABCF2. (A and B) Accumulation of ACTN4, but not ABCF2, in the RIPA-insoluble fraction with $0.5 \mathrm{mM} \mathrm{H}_{2} \mathrm{O}_{2}$ stimulation. Five micrograms of RIPA-soluble and RIPAinsoluble fractions of the $100,000 \mathrm{~g}$ pellet prepared from the cells transiently overexpressing ACTN4/FLAG and ABCF2/HA were subjected to SDSPAGE and immunoblotting with anti-FLAG M2 mAb and anti-HA and anti-caveolin-1 antibodies. (A) Immunoblots. ACTN4/FLAG, ABCF2/HA, and caveolin-1 were detected. Molecular weight markers (in kilodaltons) are indicated on the left. (B) Densitometric analysis of the immunoblot data shown in (A). The ratio of the ACTN4/FLAG contents (normalized with caveolin-1 content) in the presence of $0.5 \mathrm{mM} \mathrm{H}_{2} \mathrm{O}_{2}$ to that in the absence of $0.5 \mathrm{mM}$ $\mathrm{H}_{2} \mathrm{O}_{2}$ is plotted. $* \mathrm{P}<0.05$ (significant difference between the data from cells with or without $\mathrm{H}_{2} \mathrm{O}_{2}$ stimulation). (C and D) $\mathrm{H}_{2} \mathrm{O}_{2}$ stimulation enhances the interaction between ACTN4 and ABCF2 in the RIPA-insoluble fraction. (C) RIPA-soluble and RIPA-insoluble proteins from the 100,000g pellets were immunoprecipitated using anti-FLAG M2 mAb and were immunoblotted with anti-FLAG mAb and anti-HA antibody (right). Transfected ACTN4/FLAG and ABCF2/HA were shown in the input (left). (D) Densitometric analysis of the immunoblot data shown in (C). The ratio of the coprecipitated ABCF2/HA content (normalized with the precipitated ACTN4/FLAG content) with $\mathrm{H}_{2} \mathrm{O}_{2}$ stimulation (black columns) to that of control (white columns) is plotted. $* P<0.05$ (significant difference between the data from cells with or without $\mathrm{H}_{2} \mathrm{O}_{2}$ stimulation). Experimental conditions are the same as reported previously (Ando-Akatsuka et al., 2012). HA, hemagglutinin; IB, immunoblotted; IP, immunoprecipitated; mAb, monoclonal antibody; RIPA, radioimmunoprecipitation assay buffer.

5'-3-O-(thio)triphosphate $(\mathrm{GTP} \gamma \mathrm{S})$ (Doroshenko et al., 1991; Doroshenko and Neher, 1992; Nilius et al., 1994a; Shen et al., 1996; Mitchell et al., 1997b; Estevez et al., 2001) or by a reduction in intracellular ionic strength (Cannon et al., 1998; Nilius et al., 1998; Voets et al., 1999; Sabirov et al., 2000). However, it is not known how these maneuvers lead to activation of VSOR currents. Since intracellular introduction of GTP $\gamma \mathrm{S}$ may make a great impact on $\mathrm{G}$ protein-coupled receptors and small G proteins, especially Rho family members, which regulate actin cytoskeleton dynamics (Pedersen et al., 2001), GTP $\gamma$ S may thereby affect both type A and type $B$ mechanisms for VSOR activation. Reduced ionic strength should increase the surface potential on a number of intracellular proteins, which possess multivalent negative charges and may thereby affect the dual mechanisms of VSOR activation. Since F-actin is known to be disrupted at lower ionic strength (Senger and Goldmann, 1995; Miyata et al., 1997), it is conceived that swelling-induced VSOR activation by reduced ionic strength is mediated by actin disassembly, which was reported to enhance VSOR activation (Levitan et al., 1995; Okada, 1997). In this regard, VSOR activation was actually found to be induced without cell swelling or even in shrunken cells under conditions of very low ionic strength (Cannon et al., 1998). However, it must be noted that swelling-induced activation of VSOR is not solely accounted for by reduced ionic strength, because VSOR can be robustly activated by inflating cells by forcing isotonic fluid with identical ionic strength into them through the patch pipette under the whole-cell configuration (Hagiwara et al., 1992; Doroshenko, 1998; Best and Brown, 2009). Recently, VSOR-like anion channel activity was found to be activated by reducing 
ionic strength when purified LRRC8A proteins were reconstituted together with LRRC8D or LRRC8E (Syeda et al., 2016). There is a possibility that this channel activation in response to reduced ionic strength was caused by modification of charge-mediated interaction between the LRR domains of LRRC8A and 8D or $8 \mathrm{E}$. However, this channel activity is somewhat different from the native VSOR activity, in light of its small single-channel conductance (approximately $8 \mathrm{pS}$ ), independence of intracellular ATP, and lack of inactivation kinetics. Such nonspecific activating effects of reduced ionic strength were also found on non-VSORtype anionic currents residually observed in neutrophils isolated from ebo/ebo mice in which LRRC8A truncation mutants naturally occur (Behe et al., 2017).

The swelling-induced VSOR current was potentiated by cytosolic cAMP produced by stimulation of Gscoupled receptors such as the $\mathrm{Ca}^{2+}$-sensing receptor (Shimizu et al., 2000) and the vasopressin $\mathrm{V}_{2}$ receptor (Sato et al., 2011). Although the mechanism by which cAMP potentiates VSOR activity is not known precisely, there is a possibility that cAMP modulates cytoskeleton rearrangement, thereby indirectly augmenting VSOR activity by affecting the ACTN4-ABCF2 interaction (Fig. 4).

3. Molecular Identity. The molecular identification of VSOR has passed through various vicissitudes of fortune. For the VSOR protein, at least five candidates, including P-glycoprotein, $\mathrm{p} I_{\mathrm{Cln}}, \mathrm{ClC}-3$, bestrophin-1 (Best1), and some transmembrane TMEM16/anoctamin (ANO) members (particularly TMEM16F/ANO6), have been proposed thus far but were dismissed, almost one after another, by successive works performed in a large number of laboratories (see reviews by Nilius et al., 1997b; Okada, 1997; Nilius and Droogmans, 2001; Jentsch et al., 2002; Okada et al., 2009b, 2018; Pedersen et al., 2016). One of the essential components of VSOR was finally discovered in 2014 (Qiu et al., 2014; Voss et al., 2014).

Using an unbiased genome-wide siRNA screening method with an $\mathrm{I}^{-}$-sensitive fluorescence-based assay, two independent groups simultaneously reported that a membrane-spanning protein (LRRC8A) acts as an essential component of VSOR molecular identity in human cell lines and T lymphocytes (Qiu et al., 2014; Voss et al., 2014). Both groups convincingly showed that knockdown of human LRRC8A (hLRRC8A) abrogated swelling-induced VSOR currents and taurine release, thereby suppressing RVD in several human cell lines. In addition, the authors demonstrated that transfection of hLRRC8A cDNA rescued VSOR activity in stable LRRC8A knockdown cells, indicating that hLRRC8A plays an essential role in human VSOR activity. Subsequently, knockdown of mouse LRRC8A was also demonstrated to greatly reduce VSOR activity in a mouse C127 cell line (Okada et al., 2017) and in primary cultured mouse nodose ganglia neurons (Wang et al., 2017b). More recently, mouse VSOR activity was found to be drastically diminished in T cells from $\operatorname{Lrc} 8 a^{-1-}$ mice and ebo/ebo mice with a $L r r c 8 a$ frameshift mutation (Platt et al., 2017) and in neutrophils from ebo / ebo mice (Behe et al., 2017). Thus, it is evident that LRRC8A is an essential core component of VSOR not only in human cells but also in rodent cells. This notion was indirectly supported by the following three studies on swelling-induced osmolyte release, which is shown to be mediated via VSOR. First, siRNA-mediated knockdown of LRRC8A reduced the swelling-induced release of aspartate, glutamate, and taurine from rat astrocytes (Hyzinski-García et al., 2014). Second, a reduction in swelling-induced taurine release in cisplatin-resistant human ovarian cancer A2780 cells correlated with reduced expression of LRRC8A (Sørensen et al., 2014). Third, the swelling-induced release of neurotransmitters including glutamate, D-aspartate, D-lysine, D-serine, GABA, and taurine was observed in wild-type human embryonic kidney 293 (HEK293) cells but was abolished in $L R R C 8 A^{-1-}$ HEK293 cells (Lutter et al., 2017). Such a role of LRRC8A in anion channel formation appears to be specific to VSOR activity, because no significant effect of LRRC8A knockdown was observed on ASOR, Maxi-Cl, CaCC, and CFTR currents in mouse C127 cells (Okada et al., 2017).

The LRRC8 family consists of five members (LRRC8A-8E), and LRRC8D was shown to directly interact with LRRC8A, 8B, and $8 \mathrm{C}$ by immunoprecipitation studies coupled to mass spectrometry (Lee et al., 2014). In addition, sequential coimmunoprecipitation studies provided evidence for the interaction between LRRC8A, LRRC8C, and LRRC8E (Lutter et al., 2017). Hence, there is a possibility that LRRC8A forms heteromers with four other LRRC8 members, thereby participating in VSOR activity (Stauber, 2015). In fact, VSOR currents in human HCT116 and HEK293 cells lacking all five LRRC8 genes were rescued by expressing LRRC8A together with LRRC8C or 8E (Voss et al., 2014). Also, endogenous VSOR activity in HeLa cells was abolished by quadruple knockdown of LRRC8B-8E (Sato-Numata et al., 2017). These data suggest that the VSOR heteromer consists of LRRC8A together with LRRC8C, 8D, and/or 8E. Supporting this hypothesis, the swelling-induced release of aspartate from rat astrocytes was largely suppressed by not only double knockdown of LRRC8C and 8D but also by double knockdown of LRRC8A and 8E (Schober et al., 2017). Accumulating evidence has shown that LRRC8B may not be requisitely involved in VSOR activity, as follows. First, when LRRC8B was transfected together with LRRC8A in HCT116 cells with disruption of all five LRRC8 genes (LRRC8A ${ }^{-1-}$ cells), VSOR activity was not restored under hypotonic conditions (Voss et al., 2014). Second, when Xenopus oocytes, which virtually lack all LRRC8 genes and endogenous VSOR activity, were coinjected with LRRC8A with $8 \mathrm{~B}$ or that of 
LRRC8A tagged with fluorescent proteins $\left(8 \mathrm{~A}^{*}\right)$ and $8 \mathrm{~B}^{*}$, hypotonic stimulation failed to activate VSOR currents, although coinjection of LRRC8A plus $8 \mathrm{E} / 8 \mathrm{D}$ or of $8 \mathrm{~A}^{*}$ plus $8 \mathrm{C}^{*} / 8 \mathrm{D}^{*} / 8 \mathrm{E}^{*}$ produced VSOR activity (Gaitán-Peñas et al., 2016). In addition, VSOR activity was not induced by coinjection of $8 \mathrm{~A}^{*}$ with $8 \mathrm{~B}^{*}$ even after application of a membrane-permeable oxidizing agent, whereas VSOR currents induced by coinjection of $8 \mathrm{~A}^{*}$ plus $8 \mathrm{C} * / 8 \mathrm{D} * / 8 \mathrm{E} *$ were, in contrast, greatly affected by oxidation (Gradogna et al., 2017). Third, triple knockdown of LRRC8C, $8 \mathrm{D}$, and $8 \mathrm{E}$ abolished endogenous VSOR activity in HeLa cells, which endogenously express all five LRRC8 genes (Sato-Numata et al., 2017). Fourth, disruption of LRRC8C, 8D, and 8E abolished endogenous VSOR activity in HEK293 cells; thus, the remaining LRRC8A and $8 \mathrm{~B}$ failed to maintain VSOR activity, whereas LRRC $8 \mathrm{~A}$ and $8 \mathrm{C} / 8 \mathrm{D} / 8 \mathrm{E}$ could largely express VSOR activity (Lutter et al., 2017). Thus, it is likely that LRRC8B plays some role other than a VSOR component. In fact, LRRC8B was recently reported to be localized in the ER and was associated with ER calcium leakage in HEK293 cells (Ghosh et al., 2017).

By qualitatively evaluating the ratio of currents at the end to beginning of a 2 -second pulse $\left(I_{2 \mathrm{sec}} / I_{\max }\right)$, Jentsch's group observed that the VSOR currents in LRRC8 ${ }^{-1-}$ HCT116 cells coexpressing LRRC8A with LRRC8E exhibited faster inactivation kinetics upon application of depolarizing pulses, whereas those of the cells coexpressing LRRC8A with LRRC8C appeared to inactivate much more slowly (Voss et al., 2014). Thereafter, they showed that the C-terminal part of the first extracellular loop of LRRC8E determines the inactivation kinetics of VSOR currents in this cell line (Ullrich et al., 2016). These data suggest that LRRC8E per se or its associated subcomponent represents the inactivation gate or its accelerating factor, whereas LRRC8C per se or its associated subcomponent acts as the decelerating factor. In contrast to HCT116 cells, inactivation kinetics more accurately evaluated by the half-maximal inactivation time in HeLa cells were significantly hastened by siRNA-mediated knockdown of hLRRC8A but not of other LRRC8 members, even including LRRC8E and 8C, whereas the magnitude of VSOR currents was, at the same time, significantly suppressed by knockdown of LRRC8A but was not affected by other LRRC8 members (Sato-Numata et al., 2017). This fact suggests that LRRC8A per se or its associated regulatory subcomponent serves as a decelerating factor for VSOR inactivation in HeLa cells. In this cell line, knockdown of any single LRRC8 isoform also failed to retard the inactivation rate (Sato-Numata et al., 2017), suggesting that an as-yet-unidentified accelerating factor or subcomponent other than LRRC8 members is involved in the inactivation kinetics of VSOR currents.
Taken together with the data obtained by Voss et al. (2014), it is natural to deem that any LRRC8 member per se does not provide the inactivation gate for VSOR, and that some subcomponents associated with the pore-forming core components may participate in the inactivation gate or act as the accelerating and decelerating for the gate. In any case, any mutational changes in the inactivation kinetics may not provide direct evidence for an involvement of this molecule in the channel pore per se. Qiu et al. (2014) showed that VSOR activity in HeLa cells expressing the T44C mutant of LRRC8A in which threonine-44 was substituted with cysteine was strongly suppressed by a membrane-impermeable, thiol-reactive reagent sodium (2-sulfonatoethyl)methanethiosulfonate, and this mutation increased VSOR permeability to $\mathrm{I}^{-}$. However, the substitutions of threonine at position 44 by glutamate and arginine (T44E and T44R), which confer negative and positive charges, respectively, on this site unexpectedly had little effect on the permeability ratio of $\mathrm{I}^{-}$to $\mathrm{Cl}^{-}$through the channel (Qiu et al., 2014). Also, combinatory expression of charge-neutralizing mutants for K98 (K98A or K98N) of LRRC8A and for K91 (K91A or K91N) of LRRC8E failed to affect the sequence of $\mathrm{I}^{-}>\mathrm{Cl}^{-}$permeability of VSOR currents in LRRC $8^{-/-}$ HCT116 cells (Ullrich et al., 2016). Furthermore, charge-reversal mutants for K98 (K98E) of LRRC8A, for K100 (K100E) of LRRC8C, and for K91 (K91E) of LRRC8E did not affect anion-to-cation selectivity but only led to a small change in the degree of $\mathrm{I}^{-}$and $\mathrm{Cl}^{-}$ permeability while maintaining the same $\mathrm{I}^{-}>\mathrm{Cl}^{-}$ permeability sequence (Ullrich et al., 2016). Thus, these observations rather suggest that T44 and K98 of LRRC8A, K100 of LRRC8C, and K91 of LRRC8E are not located at the anion-selective filter within the VSOR pore. However, different heteromeric combinations of LRRC8A together with LRRC8C, 8D, or 8E may influence VSOR pore conformation, thereby only slightly affecting the pore properties including single-channel conductance, $\mathrm{I}^{-}>\mathrm{Cl}^{-}$permeability, outward rectification, and open probability in the reconstituted system (Syeda et al., 2016). More recently, LRRC8 subunit composition was found to determine the selectivity of VSOR to organic substrates such as cisplatin and taurine (Planells-Cases et al., 2015) as well as GABA, taurine, and myo-inositol (Lutter et al., 2017). Before firmly judging these facts as evidence of pore formation by LRRC8, however, it is necessary to verify that the pathway for these neutral osmolytes is identical to that for anions.

Our recent microarray studies and Western blot analyses showed that the expression level of LRRC8A mRNA and protein in human VSOR-deficient KCP-4 cells is not much different from that in parental VSORrich KB cells and human VSOR-rich HEK293T, HeLa, and Intestine 407 cells (Okada et al., 2017). In addition, we noted that expression levels of LRRC8D and $8 \mathrm{E}$ 
mRNAs in VSOR-deficient KCP-4 cells are not significantly different from the above-mentioned four other human VSOR-rich cell lines (Okada et al., 2017). Furthermore, expression levels of LRRC8B and 8C mRNAs in KCP-4 cells are rather higher than those in these VSOR-rich cell lines (Okada et al., 2018). Thus, there still remains a possibility that some molecule other than LRRC8 members serves as a main component forming the VSOR pore. In this regard, it should be noted that overexpression of LRRC8A rather gave rise to great suppression of endogenous VSOR currents in human cells (Qiu et al., 2014; Voss et al., 2014) and mouse cells (Okada et al., 2017), suggesting that excess LRRC8A expression induces a dominant negative effect caused by the formation of complexes exerting a poreclosing or pore-disrupting action (Okada et al., 2017). Moreover, LRRC8A overexpression together with LRRC8C failed to enhance endogenous VSOR currents (Voss et al., 2014). This fact may represent firm evidence that there is some as-yet-unidentified nonLRRC8 core component (X) for VSOR. In addition, anionic currents of the channels reconstituted with LRRC8A plus 8D or $8 \mathrm{E}$ were independent of intracellular ATP and did not exhibit voltage-dependent inactivation kinetics (Syeda et al., 2016). Thus, this missing core component is additionally required to reproduce intracellular ATP dependence and voltage-dependent inactivation kinetics, which are phenotypical characteristics of VSOR currents. In HeLa cells, knockdown of each LRRC8 member did not abolish or slow the voltage-dependent inactivation kinetics of VSOR currents (Sato-Numata et al., 2017), again suggesting that some other core component $\mathrm{X}$ exists that is essentially involved in or associated with the inactivation gate in this human epithelial cell line.

An involvement of NOX activity in the mechanism of VSOR activation may raise the possibility that some member of the NOX family represents the missing core component X. Supporting this hypothesis, LRRC8A (a core component of VSOR) was recently found to colocalize and interact with NOX1 in primary aortic smooth muscle cells (Choi et al., 2016). In addition, VSOR activation was suggested to be dependent in part on the assembly of an active NOX complex on the basis of suppressing effects of an inhibitor of NOX assembling, 4-(2-aminoethyl)-benzenesulfonyl fluoride, on VSOR currents in human neutrophils (Ahluwalia, 2008a). Seven members of the NOX family (NOX1-NOX5 and dual oxidases DUOX1 and DUOX2) share the capacity to transport electrons across the plasma membrane and generate superoxide and other downstream ROS (Bedard and Krause, 2007). However, microarray assays showed that expression levels of mRNAs for NOX1, NOX3, NOX4, DUOX1, and DUOX2 in VSOR-rich human epithelial cell lines (HeLa, intestine 407, KB, and HEK293T) were very low (unpublished data). Moreover, when NOX1, NOX2, NOX4, and NOX5 were overexpressed in HEK293T cells, swelling-induced VSOR currents were not significantly affected, as shown in Fig. 6. These facts may preclude the above possibility.

The following criteria for the molecular identification of the main component of VSOR were previously proposed (Okada et al., 1998). First, transfection with the gene for the candidate protein induces swellingactivated anionic currents with characteristics identical to those of phenotypic properties of VSOR. Second, cells functionally exhibiting the VSOR current endogenously express the candidate mRNA and protein. Third, elimination of the candidate protein expression abolishes the endogenous VSOR current. Fourth, mutation of the candidate gene gives rise to significant changes in the important pore properties of the channel, such as singlechannel conductance, rectification, and anion or anion/ cation selectivity. A fifth criterion can also be proposed: channel activity exhibiting all of the phenotypic properties (see section II.B.1) must be reconstituted by incorporating the candidate protein(s) in the lipid bilayer. Although the essential component of VSOR has now been identified as LRRC8A plus LRRC8C/8D/8E (Voss et al., 2014), findings showing that this combinatory expression did not reproduce VSOR activity (Voss et al., 2014; Okada et al., 2017), that charge-modifying mutation of LRRC8A did not markedly alter the pore properties (Voss et al., 2014), and that VSOR-like activity produced by the reconstitution system lacked intracellular ATP dependence and inactivation kinetics (Syeda et al., 2016) fail to meet the above criteria 1,4 , and 5 , respectively. Further investigation is thus required before the molecular identities of the main components of VSOR, which form the pore, are precisely determined.

Since the LRR motif is known to mediate proteinprotein interactions (Kobe and Kajava, 2001), it is highly possible that LRRC8A can interact not only with other paralogs of the LRRC8 family but also with other proteins, which may include the VSOR pore-forming protein and/or its regulator protein. In fact, VSOR activity was drastically diminished when the 15 terminal LRRs of LRRC8A were truncated (Platt et al., 2017). In addition, the number of subunits in the LRRC8 heteromer containing LRRC8A and $8 \mathrm{E}$ was greater than six (Gaitán-Peñas et al., 2016). Furthermore, LRRC8A was shown to physically interact with TMEM16A/ANO1 and thereby regulate ANO1-mediated CaCC currents in Xenopus oocytes (Benedetto et al., 2016). In light of these facts, there remains a possibility that LRRC8A and/or its paralog(s) interacts with an additional or genuine VSOR pore-forming protein other than LRRC8 members. Since most of the LRRcontaining protein families are known to share common functions such as an organizing role in intracellular $\mathrm{Ca}^{2+}$ signaling cascades (Abascal and Zardoya, 2012), it might be possible that LRRC8A somehow participates 

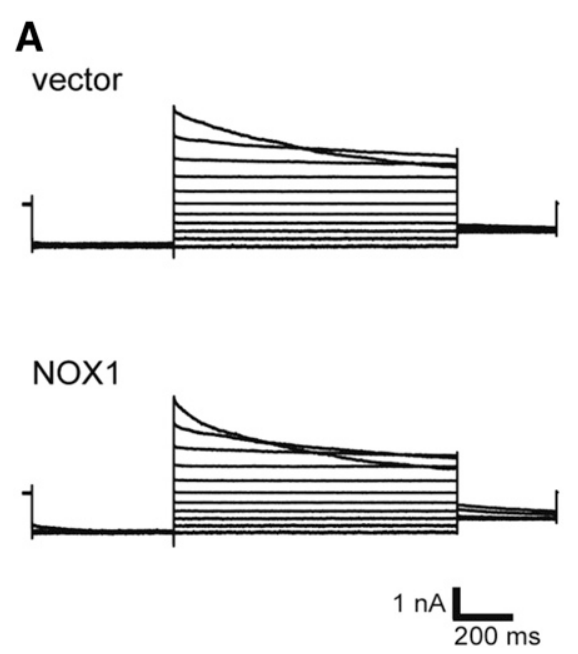

B

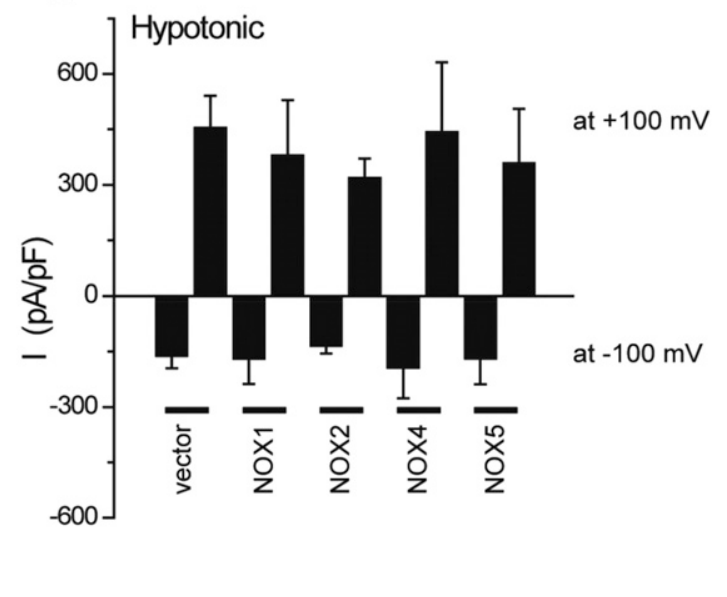

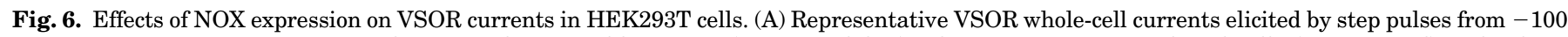

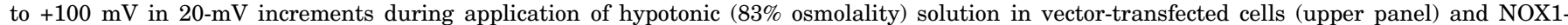

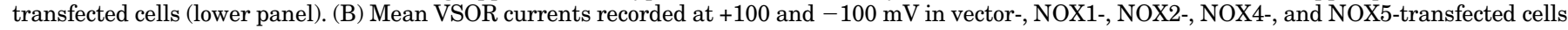
$(n=5-14)$. Experimental conditions are the same as reported previously (Okada et al., 2009a; Ando-Akatsuka et al., 2002).

in the $\mathrm{Ca}^{2+}$-dependent type $\mathrm{B}$ mechanism for VSOR activation (Fig. 4).

Finally, it is noteworthy that a girl carrying a C-terminal truncated variant of LRRC8A, from whom the LRRC8 gene was first identified, was able to survive with only congenital agammaglobulinemia and minor facial anomalies (Sawada et al., 2003). Furthermore, LRRC8A knockout mice are not always embryonically lethal; some could be born alive, although they exhibit a variety of abnormalities (Kumar et al., 2014), in contrast to the indispensable roles of VSOR in cell survival and proliferation in all mammalian tissues. Moreover, clustered regularly interspaced short palindromic repeatsbased screens showed that LRRC8A and $8 \mathrm{C} / 8 \mathrm{D} / 8 \mathrm{E}$ are not essential genes for proliferation and survival in human cancer cell lines (Wang et al., 2015). In addition, a recent study showed that swelling-induced VSOR-like currents were not suppressed, but rather were enhanced, by shRNA-mediated knockdown of LRRC8A in human retinal pigment epithelium (RPE) cells derived from induced pluripotent stem cells from a healthy donor (Milenkovic et al., 2015). These facts, on balance, may even suggest the possibility that LRRC8 members are necessary regulators for other VSOR pore-forming proteins. To elucidate whether the VSOR pore is formed by LRRC8 per se, researchers must verify that its charge-modifying mutant exhibits transition of the anion selectivity sequence from Eisenman type I to another Eisenman type or from anion selective to cation permeable or cation selective. Also, it must be pointed out that the channels reconstituted with the full set of core components should exhibit phenotypical properties including intracellular ATP requirements and intracellular free $\mathrm{Mg}^{2+}$ sensitivity.

To note, this article was submitted after the publication of several articles that show the hexameric structure of LRRC8A, mainly using cryo-electron microscopy
(Deneka et al., 2018; Kasuya et al., 2018; Kefauver et al., 2018), and suggest an involvement of LRRC8 in the VSOR pore using $L R R C 8^{-/-}$HEK293 cells (Deneka et al., 2018) and HCT116 cells (Yamada and Strange, 2018; Zhou et al., 2018). Deneka et al. (2018) showed that the hexameric structure, which incorporates a pore-like, vase-shaped conformation, can be formed by purified recombinant LRRC8A alone or together with LRRC8C. In addition, the charge-neutralizing R103A mutant slightly increased the cation permeability of LRRC8A/8C heteromers, although the general preference of anions was still maintained, suggesting that the first extracellular loop is involved in the anion selectivity filter of VSOR (Deneka et al., 2018). However, at variance with this inference, a positive chargeconferring L105R mutant of LRRC8C failed to affect the anion selectivity of LRRC8A/8C heteromers. Yamada and Strange (2018) showed that a 25-aminoacid sequence unique to the intracellular loop of LRRC8A plays a role in VSOR pore structure and function. On the other hand, Zhou et al. (2018) demonstrated that the $\mathrm{N}$ termini of the LRRC8A/8C heteromer participate in forming the cytosolic portion of the VSOR pore. However, changes in the anion selectivity sequence and the anion/cation permeability ratio were not observed in these studies and are to be examined in future studies.

\section{Maxi-Anion Channel}

Anion channels with a single-channel conductance of 200-500 pS and with $P_{\mathrm{Cl}} / P_{\mathrm{Na}}$ or $P_{\mathrm{Cl}} / P_{\mathrm{K}}>3$, called the MACs, have been observed in a wide variety of mammalian cell types (Sabirov et al., 2016), since the first observation in cultured muscle cells by Blatz and Magleby (1983). Thus far, we have observed MAC activity in mouse mammary C127 cells (Sabirov et al., 2001, 2017; Dutta et al., 2002; Sabirov and Okada, 
2004b; Toychiev et al., 2009), rabbit macula densa cells (Bell et al., 2003), rat cardiomyocytes (Dutta et al., 2004, 2008), mouse astrocytes (Liu et al., 2006, 2008b), mouse and rat fibroblasts (Sabirov et al., 2006; Okada et al., 2009b; Toychiev et al., 2009), mouse thymocytes (Kurbannazarova et al., 2011), mouse L929 fibrosarcoma cells (Islam et al., 2012), and human U937 cells (Fig. 2C) under the same experimental conditions. We found that all of these cells share phenotypical properties, as follows: 1) single-channel conductance of 300$500 \mathrm{pS}, 2)$ a linear (nonrectifying) current-voltage (I-V) relationship for instantaneous currents, 3) voltagedependent inactivation kinetics at more positive and negative potentials than approximately $\pm 20 \mathrm{mV}$, and 4) high anion-to-cation selectivity without sensitivity to cation substitution or with $P_{\mathrm{Cl}} / P_{\mathrm{K}}$ or $P_{\mathrm{Cl}} / P_{\mathrm{Na}}$ of $\geq 6$. In addition, all of the large-conductance anion channels studied in our laboratory exhibited sharp sensitivity to a trivalent cation gadolinium $\left(\mathrm{Gd}^{3+}\right)$. Our previous critical analysis of all of the properties of largeconductance anion channels hitherto reported by our laboratory and others indicated that the channels reported in $61 \%$ of the original articles (51 of 84), including the first study (Blatz and Magleby, 1983), showed the above-described four phenotypical properties and those in $26 \%$ of the articles ( 22 of 84 ) were close to these properties (Sabirov et al., 2016). Thus, the MAC of this particular fingerprint was designated as "Maxi-Cl" (Sabirov et al., 2016).

Maxi-Cl is activated by osmotic swelling (Strange et al., 1996; Sabirov and Okada, 2009; Sabirov and Merzlyak, 2012; Sabirov et al., 2016) and therefore belongs to the VAAC group. As one of the VAACs, Maxi-Cl was expected to provide a $\mathrm{Cl}^{-}$efflux pathway during the RVD process (Falke and Misler, 1989; Schlichter et al., 1990; Jalonen, 1993; Mitchell et al., 1997a) and the AVD process (Elinder et al., 2005). Maxi-Cl was also suggested to participate in the epithelial transport of $\mathrm{Cl}^{-}$(McGill et al., 1993; Do et al., 2004) and $\mathrm{HCO}_{3}^{-}$(Schneider et al., 1985; Becq et al., 1992; Riquelme et al., 2004) and in the charge balance in $\mathrm{K}^{+}$uptake in Schwann cells (McLarnon and Kim, 1991; Quasthoff et al., 1992). Our studies demonstrated that Maxi-Cl serves as a conductive pathway for the regulated release of ATP from a number of cell types (Sabirov et al., 2001, 2017; Dutta et al., 2002, 2004, 2008; Bell et al., 2003; Liu et al., 2008a,b; Islam et al., 2012) and glutamate from astrocytes (Liu et al., 2006), thereby playing a role in cell-to-cell signaling and being classified as a VSOAC.

1. Phenotypical Properties. First, Maxi-Cl is clearly distinguished from other anion channels by its very large unitary conductance (300-500 pS) under physiologic conditions. Multiple subconductance states have often been observed in the unitary events of Maxi-Cl (Bolotina et al., 1987; Bosma, 1989; Becq et al., 1992; Olesen and Bundgaard, 1992; Brown et al., 1993; Sun et al., 1993; Dutta et al., 2004). The large single-channel conductance of Maxi-Cl implies its wide pore size. The fact that ATP anions with a mean diameter of 1.2-1.3 nm produce a fast open-channel block of Maxi-Cl (Sabirov et al., 2001) suggests that the diameter of its selectivity filter is close to or just slightly larger than $1.3 \mathrm{~nm}$. Furthermore, permeability studies of Maxi-Cl to organic anions of different sizes in $\mathrm{C} 127$ cells suggested that the pore diameter at the narrowest portion is 1.1-1.5 nm (Sabirov and Okada, 2004b). In addition, our studies conducted using the nonelectrolyte partitioning method in $\mathrm{C} 127$ cells showed that Maxi-Cl appears to have a wider extracellular entrance with a diameter of approximately $2.8 \mathrm{~nm}$ and a narrower intracellular entrance with a diameter of $2.3 \mathrm{~nm}$ (Sabirov and Okada, 2004b).

The second phenotypical property of Maxi-Cl is its ohmic behavior, which distinguishes it from other VAACs (i.e., outwardly rectifying VSOR and inwardly rectifying ClC-2). The $I-V$ relationship of the fully open state of unitary events is symmetrical and linear with no rectification (Fig. 2C). Even in macropatches, the voltage dependence of the instantaneous currents was linear in C127 cells (Sabirov et al., 2001) and rat cardiomyocytes (Okada et al., 2009b). However, the steady-state macropatch currents and whole-cell currents exhibited sigmoidal $I-V$ relationships because of voltage-dependent inactivation.

The third phenotypic property of Maxi-Cl is its voltage- and time-dependent inactivation at more positive and negative potentials than approximately \pm $20 \mathrm{mV}$ (Fig. 2C). In other words, the channel stays open at 0 to $\pm 20 \mathrm{mV}$ but exhibits voltage-dependent gating with rapid closing when the membrane potential exceeds approximately $\pm 20 \mathrm{mV}$. This property reflects the bell-shaped dependence of Maxi-Cl open channel probability, with the maximum at a certain voltage near $0 \mathrm{mV}$ in a variety of cell types (Bosma, 1989; Hals et al., 1989; Jalonen et al., 1989; Schlichter et al., 1990; McGill et al., 1992; Pahapill and Schlichter, 1992; Sun et al., 1992, 1993; Bettendorff et al., 1993; Brown et al., 1993; Forshaw et al., 1993; Jalonen, 1993; Hardy and Valverde, 1994; Mitchell et al., 1997a; Riquelme and Parra, 1999; O'Donnell et al., 2001; Sabirov et al., 2001; Do et al., 2004; Riquelme et al., 2004; Elinder et al., 2005; Akanda and Elinder, 2006).

The fourth phenotypic property of Maxi-Cl is its strong anion selectivity. The $I-V$ relationship and the reversal potential of Maxi-Cl currents are often unaffected by substitution of a large cation such as $N$-methyl-D-glucamine ${ }^{+}$or $\mathrm{TEA}^{+}$for $\mathrm{Na}^{+}$or $\mathrm{K}^{+}$in bathing solution. Even if Maxi-Cl exhibited very limited cation permeability, the channel well discriminates anions from cations with a very high permeability ratio of chloride over sodium or potassium $\left(P_{\mathrm{Cl}} / P_{\mathrm{Na}}\right.$ or $P_{\mathrm{Cl}} / P_{\mathrm{K}}$ ) of $20-45$ (Coulombe et al., 1987; Falke and Misler, 1989; Hals et al., 1989; McCann et al., 1989; 
Schlichter et al., 1990; Coulombe and Coraboeuf, 1992; Pahapill and Schlichter, 1992; Bettendorff et al., 1993; Mitchell et al., 1997a; Sabirov et al., 2001) or a fairly high $P_{\mathrm{Cl}} / P_{\mathrm{Na}}$ or $P_{\mathrm{Cl}} / P_{\mathrm{K}}$ value of $6-18$ (Light et al., 1990; McGill et al., 1992; Sun et al., 1992, 1993; Bajnath et al., 1993; Riquelme et al., 1995; O'Donnell et al., 2001; Do et al., 2004; De Marchi et al., 2008). Maxi-Cl exhibits weak field anion selectivity with an Eisenman type I permeability sequence of $\mathrm{I}^{-}>$ $\mathrm{Br}^{-}>\mathrm{Cl}^{-}>\mathrm{F}^{-}$(Schneider et al., 1985; Soejima and Kokubun, 1988; Bosma, 1989; Schlichter et al., 1990; Kokubun et al., 1991; Pahapill and Schlichter, 1992; Bajnath et al., 1993; Hardy and Valverde, 1994; Sabirov et al., 2001; Sabirov and Okada, 2004b; Toychiev et al., 2009).

2. Activation Mechanisms. Maxi-Cl is normally silent or inactivated but becomes activated by a variety of physiologic or pathophysiological stimuli. Its on-cell mode activation is induced by hypo-osmotic stimulation (Falke and Misler, 1989; Coulombe and Coraboeuf, 1992; Jalonen, 1993; Sabirov et al., 2001; Dutta et al., 2002, 2004, 2008; Liu et al., 2006; Kurbannazarova et al., 2011), salt stress (Bell et al., 2003), G proteincoupled receptor stimulation (Kawahara and Takuwa, 1991; Sun et al., 1992, 1993), and ischemic or hypoxic stimulation (Dutta et al., 2004; Liu et al., 2006, 2008a). Patch membrane excision is an effective stimulus to activate Maxi-Cl (Bosma, 1989; Kanno and Takishima, 1990; Light et al., 1990; Schlichter et al., 1990; Kawahara and Takuwa, 1991; Coulombe and Coraboeuf, 1992; Bajnath et al., 1993; Jalonen, 1993; Mitchell et al., 1997a; Sabirov et al., 2001, 2006; Dutta et al., 2002, 2004; Bell et al., 2003; Liu et al., 2006; Toychiev et al., 2009; Kurbannazarova et al., 2011; Islam et al., 2012).

In the cell-free excised mode, Maxi-Cl activity is independent of intracellular $\mathrm{Ca}^{2+}$ in the physiologic concentration range and is not largely affected by intracellular $\mathrm{Ca}^{2+}$ chelation (Blatz and Magleby, 1985; Soejima and Kokubun, 1988; Bosma, 1989; Falke and Misler, 1989; McCann et al., 1989; Light et al., 1990; Becq et al., 1992; Coulombe and Coraboeuf, 1992; Olesen and Bundgaard, 1992; Pahapill and Schlichter, 1992; Sun et al., 1992; Bell et al., 2003; Toychiev et al., 2009), whereas its on-cell activity is induced by the application of a Ca ${ }^{2+}$ ionophore, A23187 [5-(methylamino)2-(\{(2R,3R,6S,8S,9R,11R)-3,9,11-trimethyl-8-[(1S)-1-methyl2-oxo-2-(1H-pyrrol-2-yl)ethyl]-1,7-dioxaspiro[5.5]undec-2yl methyl)-1,3-benzoxazole-4-carboxylic acid] (Light et al., 1990; Kawahara and Takuwa, 1991; Bajnath et al., 1993; Sun et al., 1993).

Maxi-Cl activity in excised patches was activated even in the absence of cytosolic ATP (Soejima and Kokubun, 1988; McGill et al., 1992; Olesen and Bundgaard, 1992). In contrast, the unitary conductance of Maxi-Cl was reduced by $5 \mathrm{mM}$ cytosolic ATP (Bettendorff et al., 1993), and the mean number of
Maxi-Cl open channels was markedly suppressed by cytosolic Mg-ATP in a concentration-dependent manner with an $\mathrm{IC}_{50}$ of $29.2 \mu \mathrm{M}$ (Toychiev et al., 2009). A broad-spectrum serine/threonine protein kinase inhibitor, 5-(2-methylpiperazin-1-yl)sulfonylisoquinoline dihydrochloride (H7), activated Maxi-Cl-like activity in vascular smooth muscle A7r5 cells (Saigusa and Kokubun, 1988) but had no effect on Maxi-Cl activity in mammary C127 cells (Toychiev et al., 2009). In contrast, protein kinase $\mathrm{C}$ was reported to activate a non-Maxi-Cl type of MAC in renal cortical collecting duct RCCT-28 cells (Schwiebert et al., 1992). Another non-Maxi-Cl type of MAC in mouse neuroblastoma C1300 cells was inhibited by a serine/threonine protein phosphatase inhibitor, okadaic acid (Díaz et al., 2001). In contrast, Maxi-Cl activity in C127 cells subjected to hypotonic stimulation was insensitive to okadaic acid but was largely inhibited by a number of broad-spectrum protein tyrosine phosphatase inhibitors (Toychiev et al., 2009). Furthermore, even without hypotonic stimulation, protein tyrosine kinase (PTK) inhibitors brought the channel out of an inactivated state in C127 cells and mouse adult skin fibroblasts (Toychiev et al., 2009) as well as in L929 cells (Islam et al., 2012). A receptor protein tyrosine phosphatase $\zeta$ was identified to be, at least in part, responsible for activation of Maxi-Cl in mouse adult skin fibroblasts (Toychiev et al., 2009). Taken together, it appears that activation of Maxi-Cl involves protein dephosphorylation mediated by protein tyrosine phosphatases.

3. Molecular Identity. Despite a long history of research on Maxi-Cl, including its well-defined phenotypic properties and important functions, the channel's molecular nature remained undetermined until quite recently. At least four candidates had been proposed for the Maxi-Cl protein, including the plasmalemmal subtype of mitochondrial voltage-dependent anion channel, ADP/ATP carrier AAC, tweety TTYH1, and connexin 43, but all were disproved (for reviews, see Sabirov and Okada., 2005, 2009; Okada et al., 2009b, 2018). Thus, we conducted further studies by applying unbiased genome-wide approaches (Islam et al., 2013). By adopting the strategy as summarized in Fig. 7, we recently determined the molecular identity of the Maxi-Cl core component (called MAC-1) as solute carrier organic anion transporter family member SLCO2A1 (Sabirov et al., 2017). Our starting materials were membrane blebs isolated from the mouse mammary $\mathrm{C} 127$ cell line, which exhibits the highest activity of Maxi-Cl studied thus far (Sabirov et al., 2001). Functional Maxi-Cl was found to be accumulated in the constitutively activated state in the blebs. After solubilization of the total bleb-membrane proteins, we selected a fraction with the highest Maxi-Cl activity and performed a proteomics analysis by subjecting the fraction to liquid chromatography-tandem mass spectrometry, yielding 439 genes. Among them, we picked up 15 genes 

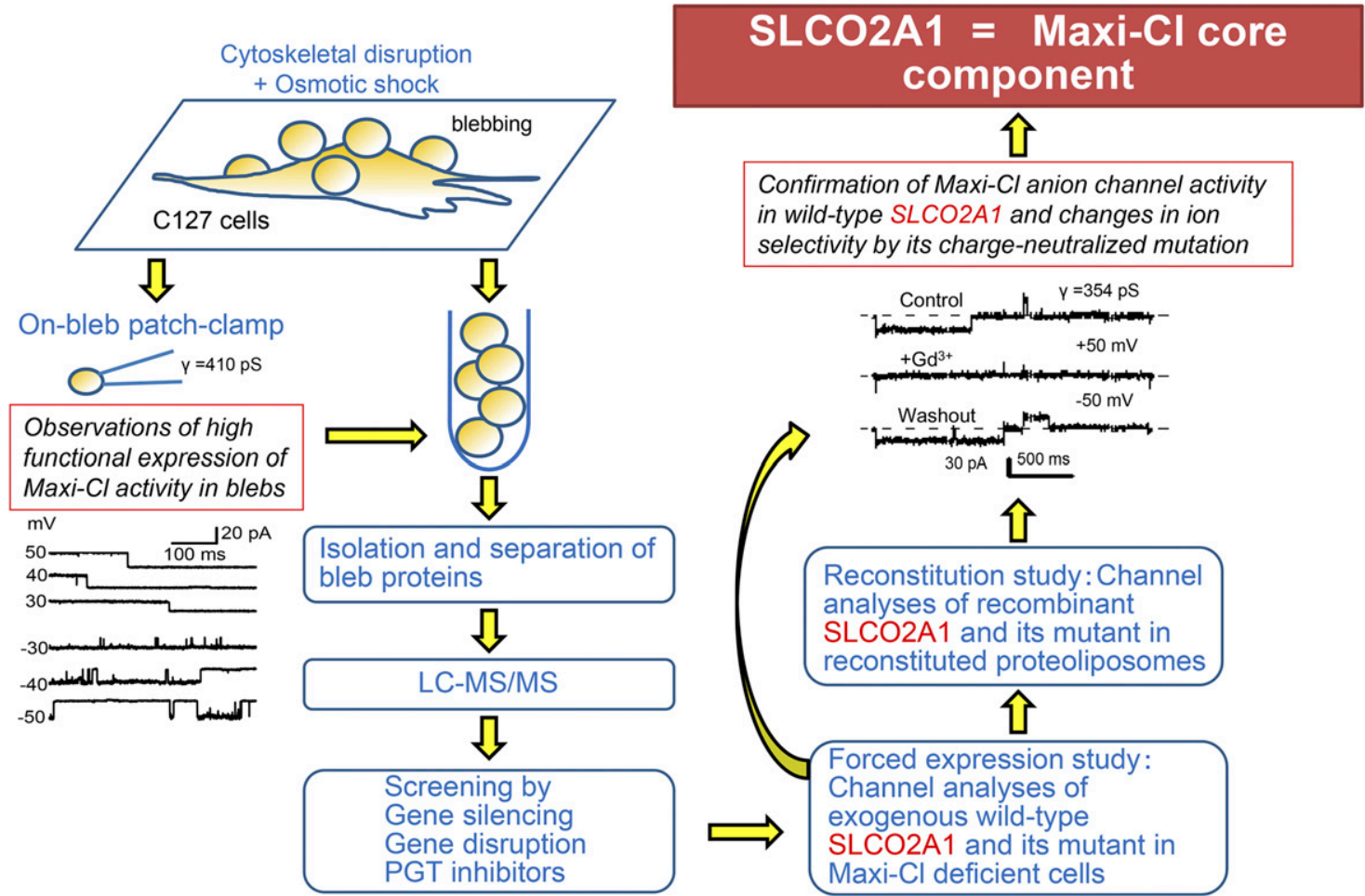

Fig. 7. Strategy adopted in our study for molecular identification of the core component of Maxi-Cl. By performing bleb membrane proteomics followed by gene silencing and disruption, mutagenesis, pharmacology, heterologous expression, and recombinant protein reconstitution, SLCO2A1 protein was identified as a core component of the ATP-conductive Maxi-Cl channel (see the text and Sabirov et al., 2017 for details). LC, liquid chromatography; MS/MS, tandem mass spectrometry.

that encode proteins with multiple transmembranespanning domains and observed the effects of their gene silencing on Maxi-Cl activity. Only siRNA- and miRNAmediated knockdown of the SLCO2A1 gene was reproducibly effective in suppressing Maxi-Cl activity. Since SLCO2A1 is known as the molecule of prostaglandin transporter (PGT), we tested the effects of PGT substrate PGE2 and PGT blockers bromosulfophthalein (BSP), bromocresol green, and indocyanine green; we found that all of these compounds could suppress Maxi-Cl currents. When SLCO2A1 was heterologously expressed in HEK293T cells, which do not endogenously express SLCO2A1 and thus lack Maxi-Cl activity, BSP-sensitive Maxi-Cl activity emerged. Mutants of SLCO2A1, G222R and P219L, which are associated with pachydermoperiostosis (Zhang et al., 2012, 2014), never emerged the channel activity, despite their successful plasmalemmal expression. On the other hand, overexpression of the charge-neutralized K613G mutant, which is known to impair SLCO2A1transporter function (Chan et al., 2002), produced channel activity with markedly reduced single-channel conductance that was relatively more selective to cations. These data suggest that SLCO2A1 is involved in the pore formation of MaxiCl. To confirm this, we then reconstituted purified recombinant SLCO2A1 proteins into lipid bilayers. Reconstituted SLCO2A1 exhibited Maxi-Cl activity that was sensitive to $\mathrm{Gd}^{3+}$ and PGE2. The charge-neutralized K613G mutant was again more permeable to cations, exhibiting a smaller single-channel conductance. Taken together, we conclude that SLCO2A1 constitutes the core component or the pore of Maxi-Cl.

In light of the above results, it is deemed that SLCO2A1 functions in two modes: 1) as a PGT in the resting state presumably with phosphorylated tyrosine residue(s) and 2) as an ATP-conductive Maxi-Cl channel in the activated state with dephosphorylated tyrosine residue(s); in addition, the transition from the resting to activated state is induced by membrane excision, cell swelling, ischemia, or hypoxia, as schematically depicted in Fig. 8. It is striking that the loss of a multigate barrier in a transporter often leads to anion channel activity, as pointed out by Minor (2017). Such bimodal channel/transporter proteins include $\mathrm{ClC}$ family members functioning as $\mathrm{Cl}^{-}$channels and $\mathrm{Cl}^{-} / \mathrm{H}^{+}$ exchangers (Picollo and Pusch, 2005; Scheel et al., 2005; Accardi and Picollo, 2010), SLC1As serving as excitatory amino acid transporters and anion channels (Cater et al., 2016; Fahlke et al., 2016), SLC26A3/6 serving as $\mathrm{Cl}^{-}$channels and anion exchangers (Ohana et al., 2009), and TMEM16F and fungal TMEM16 serving as CaCCs and lipid scramblases (Malvezzi et al., 2013; Suzuki et al., 2014; Picollo et al., 2015).

Maxi-Cl in membrane blebs derived from $\mathrm{C} 127$ cells as well as the channel reconstituted from proteins isolated from the blebs and recombinant SLCO2A1 proteins into the proteoliposomes were all in a constitutive active state (Sabirov et al., 2017), suggesting that 


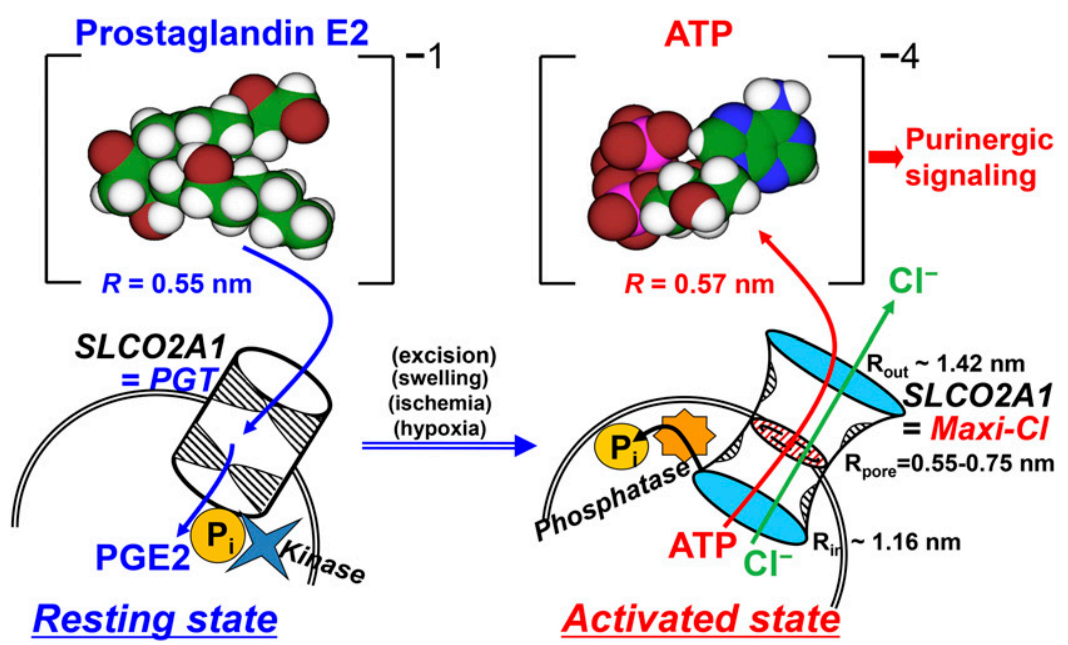

Fig. 8. Dual modes of SLCO2A1 functions as a transporter, PGT (left), and an ATP-permeable maxi-anion channel, Maxi-Cl (right).

the whole Maxi-Cl complex natively contains or is associated with some regulatory component(s) for channel activation. In addition, Maxi-Cl activity reconstituted with recombinant SLCO2A1 proteins was totally insensitive to BSP (Sabirov et al., 2017), indicating that some BSP-sensitive component is missing in the reconstituted channel. Therefore, it appears that some additional component(s) other than the core component SLCO2A1 is required for the whole Maxi-Cl complex. We must also point out that there should be some as-yet-unidentified molecules other than SLCO2A1 responsible for non-Maxi-Cl-type MACs other than MAC-1. Those missing components for Maxi-Cl and non-Maxi-Cl MAC molecules are to be identified in future studies.

\section{Volume-Correlated Anion Channels}

As noted in section I, three types of VCACs (CFTR, $\mathrm{CaCC}$, and ASOR) are activated by cAMP, $\mathrm{Ca}^{2+}$, and acidification, respectively, but not by cell volume changes. Hence, they cannot be professionally involved in cell volume regulation. Nevertheless, in some situations, they are implicated in cell volume changes.

\section{A. Cystic Fibrosis Transmembrane Conductance Regulator}

Disorder of the CFTR gene is responsible for monogenic cystic fibrosis in humans (Riordan et al., 1989). CFTR is a unique member of the ABC transporter superfamily, which functions as a low-conductance anion channel rather than as a transporter, and is expressed principally in epithelial tissues and also in some nonepithelial tissues, including cardiomyocytes and smooth muscle cells (Anderson et al., 1991; Kartner et al., 1991; for review, see Hwang and Sheppard, 2009).

CFTR is phenotypically characterized by biophysical properties exhibiting low single-channel conductance (6-10 pS), a linear $I-V$ relationship, high-field anion selectivity $\left(\mathrm{Br}^{-}>\mathrm{Cl}^{-}>\mathrm{I}^{-}\right.$: Eisenman type III selectivity sequence), and voltage independence without exhibiting any voltage-dependent activation and/or inactivation kinetics (Sheppard and Welsh, 1999). Phosphorylation of the intracellular regulatory domain (RD) mainly attained by PKA is a prerequisite to CFTR channel opening (Hegedus et al., 2009). Recent studies using electron cryomicroscopy revealed that phosphorylation of RD becomes disengaged from its inhibitory position docked inside the intracellular vestibule (Liu et al., 2017; Zhang et al., 2017). Transitions between open and closed states of CFTR channels are directly induced by ATP binding and hydrolysis on the cytosolic nucleotide binding domains (NBD1 and NBD2), which can form a "head-to-tail" dimer (Kirk and Wang, 2011). CFTR was recently reported to be directly (in a manner independent of PKA) activated by membrane stretch (Zhang et al., 2010a) and osmotic cell swelling (Xie et al., 2016). The conclusions deduced in both studies were mainly based on pharmacological observations using CFTR $_{\text {inh }}-172$ (3-[(3-trifluoromethyl)phenyl]-5[(4-carboxyphenyl)methylene]-2-thioxo-4-thiazolidinone) and GlyH-101 [N-(2-naphthalenyl)-((3,5-dibromo-2,4dihydroxyphenyl)methylene)glycine hydrazide] as CFTRspecific antagonists, although these drugs were known to also block VSOR (Melis et al., 2014; Friard et al., 2017) and ClC-2 (Cuppoletti et al., 2014). Moreover, these reports did not provide enough biophysical evidence to convince the readers that recorded channel events exhibit the phenotypic characteristics of CFTR. Thus, at present, CFTR cannot be classified as a VAAC.

CFTR is a multifunctional protein that functions not only as an anion channel but also as a regulator for a number of other ion channels (Kunzelmann and Schreiber, 1999; Schwiebert et al., 1999). For instance, CFTR regulates the epithelial amiloride-sensitive $\mathrm{Na}^{+}$ channel ENaC (Kunzelmann et al., 1997; Schreiber et al., 1999), renal outer medullary $\mathrm{K}^{+}$channel 2 (McNicholas et al., 1997), and the outwardly rectifying 
(depolarization-induced) $\mathrm{Cl}^{-}$channel (Fulmer et al., 1995; Jovov et al., 1995) through protein-protein interactions mediated by NBD1, as well as CaCC mediated by RD (Wei et al., 2001) and VSOR mediated by NBD2 (Ando-Akatsuka et al., 2002).

Activation of CFTR in epithelial cells causes $\mathrm{Cl}^{-}$ secretion and thus often leads to cell shrinkage, called a secretory volume decrease (Manabe et al., 2004). For example, daidzein-induced activation of CFTR resulted in shrinkage of renal epithelial cells due to a decrease in $\left[\mathrm{Cl}^{-}\right]_{\mathrm{i}}$ (Marunaka, 1997). However, genistein-induced CFTR activation did not result in cell shrinkage, because simultaneous activation of NKCC maintained the $\left[\mathrm{Cl}^{-}\right]_{\mathrm{i}}$ level (Niisato et al., 1999) by compensating for CFTR-mediated $\mathrm{Cl}^{-}$efflux with NKCC-mediated $\mathrm{Cl}^{-}$ influx (see Fig. 1).

A splice variant of CFTR is expressed in a variety of mammalian cardiomyocytes (Levesque et al., 1992; Hart et al., 1996; James et al., 1996) and plays roles in regulating action potential duration and establishing resting potential (McCarty, 2000). Cardiac CFTR was found to be involved in the RVD process under particular conditions in which $\beta$-adrenergic receptors were stimulated and the intracellular cAMP level was thereby increased (Wang et al., 1997; Yamamoto et al., 2001).

Cell volume regulation after a hypotonic challenge (RVD) was defective in intestinal crypt epithelial cells isolated from CFTR-knockout mice, and this was caused by the inability of $\mathrm{K}^{+}$conductance but not by CFTR $\mathrm{Cl}^{-}$ conductance (Valverde et al., 1995). In fact, a CF mutation $(\triangle \mathrm{F} 508)$ or knockdown of CFTR impaired RVD owing to a lack of swelling-induced activation of intermediate-conductance $\mathrm{Ca}^{2+}$-dependent $\mathrm{K}^{+}$channel IK1, presumably through an interaction between CFTR and potassium calcium-activated channel KCNN4 (Vázquez et al., 2001). In addition, CFTR expression was reported to facilitate the RVD process by augmenting swelling-induced ATP release mediated by the $\mathrm{Gd}^{3+}$-sensitive non-CFTR anion channel (Hazama et al., 2000; Braunstein et al., 2001). In an autocrine fashion, released ATP may stimulate purinergic P2 receptors and then induce an increase in intracellular $\mathrm{Ca}^{2+}$, thereby facilitating RVD due to activation of both $\mathrm{Ca}^{2+}$-dependent $\mathrm{K}^{+}$channels (Dezaki et al., 2000) and VSORs (Akita et al., 2011). In fact, CFTR knockout was found to abolish swelling-activated, $\mathrm{Ca}^{2+}$-activated $\mathrm{K}^{+}$channel currents (Belfodil et al., 2003) as well as VSOR currents and RVD (Barrière et al., 2003) in mouse renal tubular cells. Taken together, we conclude that noncardiac CFTR is indirectly involved in RVD efficacy.

CFTR permeates organic anions including GSH (Linsdell and Hanrahan, 1998; Kogan et al., 2003), but not ATP (Linsdell and Hanrahan, 1998), and actually mediates release of GSH in the lung (Kariya et al., 2007) and renal tubular cells (l'Hoste et al., 2010).
Phosphorylation-dependent dilation of the pore lumen with the radii of the external vestibule $(0.92 \mathrm{~nm})$ and the narrowest part $(0.69 \mathrm{~nm})$ may account for this function (Krasilnikov et al., 2011). Thus, CFTR can also be classified as a VSOAC.

\section{B. Calcium-Activated Chloride Channel}

CaCC activity was discovered in Xenopus oocytes (Miledi, 1982) and salamander photoreceptors (Bader et al., 1982). CaCC, which shows anion selectivity with an Eisenman type I sequence, was subsequently shown to be expressed in a wide variety of mammalian tissues playing fundamental roles in physiologic processes such as transepithelial transport of electrolytes and fluid, regulation of smooth muscle contractility, modulation of cardiac and neuronal action potentials, control of signaling in photoreceptors and olfactory receptors, and cancer cell proliferation (Hartzell et al., 2005; Huang et al., 2012a). CaCC opens in response to increases in the intracellular free $\mathrm{Ca}^{2+}$ concentration $\left(\left[\mathrm{Ca}^{2+}\right]_{\mathrm{i}}\right)$. The single-channel conductance of $\mathrm{CaCC}$ is approximately 8 pS (Nilius et al., 1997b; Piper and Large, 2003; Crutzen et al., 2016). CaCC currents exhibit outward rectification and voltage dependence and display activation and deactivation kinetics upon depolarization and hyperpolarization, respectively, in the physiologic range of $\left[\mathrm{Ca}^{2+}\right]_{i}$, whereas the currents become time and voltage independent and ohmic ( $I-V$ linear) at higher $\left[\mathrm{Ca}^{2+}\right]_{\mathrm{i}}$ (Frings et al., 2000; Kuruma and Hartzell, 2000).

The first candidate proposed for the molecular identity of CaCC was CLCA1, the cDNA of which was isolated in 1995 from a bovine tracheal cDNA library (Cunningham et al., 1995). However, the biophysical fingerprint of CLCA1, which lacks voltage-dependent kinetics and outward rectification and exhibits sensitivity to reducing agents such as dithiothreitol, is quite different from that of $\mathrm{CaCC}$, and there is no correlation of endogenous expression between $\mathrm{CaCC}$ activity and CLCA1 mRNA (Eggermont, 2004). Thus, CLCA1 is distinct from the molecule responsible for $\mathrm{CaCC}$, although there remains a possibility that CLCA1 is an accessory component for the CaCC complex or an activating regulator for $\mathrm{CaCC}$ (Loewen and Forsyth, 2005; Sala-Rabanal et al., 2015).

Another family, bestrophin (Best), was then proposed to confer CaCC. Human Best1 (hBest1), which was identified as the gene responsible for Best vitelliform macular dystrophy (also called Best's disease) (Marquardt et al., 1998; Petrukhin et al., 1998), was shown to function as a CaCC molecule (Sun et al., 2002). Actually, many of the disease-causing mutations in hBest1 were found to cause dysfunction of $\mathrm{CaCC}$ (Xiao et al., 2010). CaCC activity was subsequently shown to be associated with Best1 of Drosophila (Chien et al., 2006) and mice (mBest1; Park et al., 2009). In addition, similar $\mathrm{CaCC}$ activity was reported for Best2 (Qu et al., 2003b; Pifferi et al., 2006), Best3 
(Matchkov et al., 2008; O'Driscoll et al., 2008), and Best4 (Tsunenari et al., 2006). However, some properties of Best-associated $\mathrm{Ca}^{2+}$-activated $\mathrm{Cl}^{-}$currents were distinct from the classic CaCC phenotype. In particular, Best-associated currents did not show clear outward rectification and voltage-dependent activation and inactivation kinetics (Qu et al., 2003b; Chien et al., 2006; Pifferi et al., 2006; O'Driscoll et al., 2008). Moreover, endogenous $\mathrm{CaCC}$ currents were normally observed in $\mathrm{RPE}$ cells isolated from mBest1 knockout mice (Marmorstein et al., 2006) and salivary acinar cells derived from mBest2 knockout mice (Romanenko et al., 2010). Also, RPE cells derived from knock-in mice carrying the Best vitelliform macular dystrophycausing mutant (W93C) of mBest1 exhibited normal CaCC conductances (Zhang et al., 2010b). Furthermore, Best1 was recently shown to be a multifunctional protein serving as a regulator for the voltage-gated $\mathrm{Ca}^{2+}$ channel (Marmorstein et al., 2006; Rosenthal et al., 2006; Yu et al., 2008) and for $\mathrm{Ca}^{2+}$ release from the ER (Barro-Soria et al., 2010; Neussert et al., 2010; Zhang et al., 2010b; Gómez et al., 2013). On the other hand, not only Drosophila Best1 but also hBest1 and mBest2 are shown to be sensitive to changes in cell volume (Fischmeister and Hartzell, 2005; Chien and Hartzell, 2007; Xiao et al., 2009), although it remains unclear how cell volume changes are sensed by Best.

Finally, TMEM16A/ANO1 has been shown independently by three groups to exhibit a full set of the phenotypic properties of $\mathrm{CaCC}$ and to be the molecular identity of $\mathrm{CaCC}$ (Caputo et al., 2008; Schroeder et al., 2008; Yang et al., 2008). The TMEM16/ANO family has 10 members, all with 10 transmembrane domains, and plays a variety of physiologic roles in mammalian cells (Pedemonte and Galietta, 2014; Oh and Jung, 2016). TMEM16A/ANO1 shows approximately $60 \%$ sequence identity with TMEM16B/ANO2 but only $20 \%-30 \%$ sequence identity with remaining TMEM16C-K/ANO310. In fact, TMEM16B/ANO2 is also shown to be a CaCC in photoreceptors (Stöhr et al., 2009), olfactory sensory neurons (Pifferi et al., 2009; Stephan et al., 2009; Billig et al., 2011), and hippocampal neurons (Huang et al., $2012 \mathrm{~b})$. On the other hand, TMEM16A/ANO1 and TMEM16B/ANO2 were recently found to be coexpressed, exhibit molecular interaction with each other, and thereby form heteromeric channels generating CaCC currents in rat pineal glands (Yamamura et al., 2018). Although TMEM16C-G/ANO3-7 are shown to be intracellular proteins (Duran et al., 2012), TMEM16F/ANO6 exhibited CaCC activity, when overexpressed, on the plasma membrane and exhibited "noncanonical" characteristics such as lower sensitivity to intracellular $\mathrm{Ca}^{2+}$ increases (Grubb et al., 2013; Shimizu et al., 2013). On the other hand, under certain conditions, TMEM16F/ANO6, the defective mutation of which is associated with the congenital bleeding disorder Scott syndrome in humans (Suzuki et al., 2010), served as a $\mathrm{Ca}^{2+}$-dependent scramblase (Suzuki et al., 2010) and as a $\mathrm{Ca}^{2+}$-activated nonselective cation channel (Yang et al., 2012; Adomaviciene et al., 2013; Yu et al., 2015).

$\mathrm{CaCC}$ activation is often associated with cell volume changes. For example, muscarinic stimulation of intestinal epithelial cells with carbamylcholine induced fluid secretion and cell shrinkage or secretory volume decrease (Valverde et al., 1993; Manabe et al., 2004) due to CaCCmediated $\mathrm{Cl}^{-}$secretion. Stimulation with $\mathrm{Ca}^{2+}$ ionophore A23187 and neuropeptide bradykinin is known to result in swelling in platelets and glioma cells, respectively, due to $\mathrm{Cl}^{-}$influx mediated by $\mathrm{CaCC}$ activation (Fine et al., 1994; Seifert and Sontheimer, 2014). In addition, CaCC activity was also reported to be indirectly involved in the RVD process in low intracellular $\mathrm{Ca}^{2+}$ buffering conditions, because siRNA-mediated knockdown of TMEM16A/ANO1 and of TMEM16F/ANO6 slowed but did not abolish the RVD process in HEK293 and Ehrlich ascites tumor cells, respectively (Almaça et al., 2009; Juul et al., 2014). RVD was also still observed, albeit slowly, in the absence of extracellular $\mathrm{Ca}^{2+}$, although the RVD-facilitating effect of CaCC mediated by TMEM16F/ANO6 was abolished under the $\mathrm{Ca}^{2+}$-free condition (Juul et al., 2014). Thus, it is possible that volumeregulatory $\mathrm{Cl}^{-}$efflux is mediated, in part, by $\mathrm{CaCC}$. In addition, the time course of RVD was recently found to be accelerated in TMEM16F/ANO6-overexpressing HEK293 cells and slowed (but was not abolished) in intestinal epithelial cells isolated from TMEM16F/ANO6 knockout mice (Sirianant et al., 2016). Thus, it is also possible that TMEM16F/ANO6 acts as a $\mathrm{Ca}^{2+}$-activated nonselective cation channel and serves as a pathway for $\mathrm{Ca}^{2+}$ entry, which is required for volume-regulatory operation of $\mathrm{Ca}^{2+}$-activated $\mathrm{K}^{+}$channels.

\section{Acid-Sensitive Outwardly Rectifying Anion Channel}

ASOR, which is rapidly activated by extracellular acidification (at $\mathrm{pH}<6$ ), is expressed in a variety of mammalian cell types, including human epithelial HEK293 or HEK293T cells (Nobles et al., 2004; Lambert and Oberwinkler, 2005; Matsuda et al., 2010a; Okada et al., 2014; Capurro et al., 2015), HeLa cells (Wang et al., 2007; Sato-Numata et al., 2013, 2016, 2017), human red blood cells (Kucherenko et al., 2009), human monocytic leukemia THP-1 cells (Fu et al., 2013), human bronchial epithelial cell lines (Capurro et al., 2015), pancreatic adenocarcinoma CFPAC cells (Capurro et al., 2015), neuroblastoma SK-N-MC cells (Capurro et al., 2015), mouse hippocampal astrocytes (Lambert and Oberwinkler, 2005), mouse smooth muscle cells (Matsuda et al., 2010a), mouse cortical neurons (Sato-Numata et al., 2014), mouse RAW264.7 macrophages (Capurro et al., 2015), mouse and guinea pig cardiac myocytes (Yamamoto and Ehara, 2006), rat Sertoli cells (Auzanneau et al., 2003), and Fischer rat thyroid (FRT) cells (Capurro et al., 2015). This channel 
may be activated and play some physiologic or pathophysiologic roles under strong acidic conditions, such as in the inner medullary collecting duct, where the acidity of tubular filtrate can decrease below pH 5.5 (Bengele et al., 1983, 1986), and in the brain subjected to ischemia, seizure, trauma, or hyperglycemia (Kraig et al., 1985; Rehncrona, 1985; Siesjö, 1988; Chesler and Kaila, 1992).

Whole-cell ASOR current is phenotypically characterized by acid sensitivity, strong outward rectification, and activation kinetics upon application of positive voltages (Nobles et al., 2004; Lambert and Oberwinkler, 2005; Wang et al., 2007; Sato-Numata et al., 2014; Capurro et al., 2015). ASOR current was recently found to be highly sensitive to temperature, with $Q_{10}$ values of 8.8 in human epithelial HeLa cells and 5.6 in mouse cortical neurons (Sato-Numata et al., 2013, 2014). In the physiologic range of membrane potential, the single-channel conductance of ASOR is 4-7 pS in HEK293 cells (Lambert and Oberwinkler, 2005) and $4.8 \mathrm{pS}$ in HeLa cells (Wang et al., 2007). Previous studies showed that regulatory mechanisms of ASOR activation do not involve intracellular $\mathrm{Ca}^{2+}$, ATP, and G proteins (Yamamoto and Ehara, 2006) or protein kinases A and C (Capurro et al., 2015). Pharmacological studies suggested that ASOR is regulated by mitochondrion-generated ROS (Fu et al., 2013) as well as PTK and phosphatidylinositol-3-kinase (Capurro et al., 2015). In pharmacological studies, ASOR regulation was suggested to be dependent on exocytosis/endocytosis (Capurro et al., 2015), which contradicts the independence of intracellular $\mathrm{Ca}^{2+}$ and ATP.

The molecular identity of ASOR is yet to be determined. Since extracellular acidification was observed to activate anionic currents conducted via a number of $\mathrm{ClC}$ channel/transporter family members, including ClC-0 (Chen and Chen, 2001), ClC-2 (Jordt and Jentsch, 1997; Stroffekova et al., 1998), ClC-3 (Matsuda et al., 2010a), and ClC-7 (Diewald et al., 2002; Kajiya et al., 2009), it is possible that some ClC members are associated with the molecular entity of ASOR. ClC-3 (Matsuda et al., 2010a; Wang et al., 2012) and ClC-7 (Kajiya et al., 2009; Ohgi et al., 2011) were suggested to be responsible for the ASOR molecule. However, this possibility was recently ruled out in light of the following observations. First, both ClC-3 and ClC-7 were localized in the intracellular compartments but not on the plasma membrane (Auzanneau et al., 2003). Second, endogenous ASOR currents were not affected by siRNA-mediated knockdown of ClC-3 (Sato-Numata et al., 2013; Capurro et al., 2015) and ClC-7 (Wang et al., 2012; Capurro et al., 2015). Third, ASOR currents were not enhanced in cells overexpressing ClC-3a (Sato-Numata et al., 2013), ClC-3d (Okada et al., 2014), and ClC-7 (Sato et al., 2012).
The sharp outward rectification, voltage-dependent activation kinetics, and temperature sensitivity of ASOR currents are similar to those of TMEM16A/ANO1 currents. Thus, it is possible that some member of TMEM16/ANO is associated with the ASOR molecule. Inconsistently, however, ASOR currents were found to be insensitive to siRNA-mediated knockdown of not only TMEM16A but also TMEM16D, F, H, and $\mathrm{K}$ (Capurro et al., 2015).

Based on similarities in some pharmacological properties and the peak amplitudes between ASOR and VSOR currents, it was previously assumed that both anion channels share the same molecular entity with two different manifestations being exhibited (Nobles et al., 2004). However, there is a distinct difference in the time lag of activation after stimulation between immediately activating ASOR and slowly activating VSOR currents. As noted in section II.B, phenotypical properties of VSOR are distinct from those of ASOR, which include cytosolic ATP independence (Yamamoto and Ehara, 2006), cytosolic $\mathrm{Mg}^{2+}$ insensitivity (Lambert and Oberwinkler, 2005), a onedigit smaller single-channel conductance (Wang et al., 2007), and much steeper outward rectification (Auzanneau et al., 2003; Nobles et al., 2004; Lambert and Oberwinkler, 2005; Wang et al., 2007). In addition, ASOR currents are totally insensitive to a number of putative VSOR blockers, as discussed in section IV. Furthermore, both VSOR and ASOR currents were found to be simultaneously activated in an additive manner (Lambert and Oberwinkler, 2005; Yamamoto and Ehara, 2006; Matsuda et al., $2010 \mathrm{~b}$ ). Taken together, it is now clear that the molecular identity of ASOR is distinct from that of VSOR. In this regard, it is noteworthy that ASOR currents were not affected by siRNA-mediated knockdown of either LRRC8A, which is an essential component of VSOR (see section II.B), or LRRC8B/C/D/E (Sato-Numata et al., 2016).

Activation of ASOR was associated with persistent cell swelling, called necrotic volume increase (NVI), which is followed by necrotic cell death in human epithelial HeLa cells (Wang et al., 2007) and mouse cortical neurons (Sato-Numata et al., 2014) under acidotoxic conditions. The NVI process is known to be induced by water inflow driven mainly by $\mathrm{NaCl}$ influx (Barros et al., 2001; Okada et al., 2001, 2004). To obtain $\mathrm{NaCl}$ influx, some $\mathrm{Na}^{+}$permeable cation channels must be activated when anionic ASOR currents are activated, according to electroneutrality principle, thereby giving rise to depolarization, which in turn drives ASOR-mediated $\mathrm{Cl}^{-}$inflow. In HeLa cells, TRPM7 was identified to represent this cation channel activated by reduced blocking effects of extracellular divalent cations under acidotoxic conditions (Numata and Okada, 2009). In neuronal cells under acidotoxic conditions, the voltage-insensitive, acidsensitive $\mathrm{Na}^{+}$-permeable cation acid-sensing ion channel 
may represent this cationic conduction pathway to attain NVI (Xiong et al., 2004; Yermolaieva et al., 2004). Thus, ASOR is involved in cell volume changes in acidic situations in which some cation channels are simultaneously activated.

\section{Pharmacological Properties of and Distinctions between Volume-Activated Anion Channels and Volume-Correlated Anion Channels}

Pharmacological information on anion channels is important for the investigation of their properties, activation mechanisms, and functions and ultimately the development of drugs for clinical uses. In general, most anion channel blockers exhibit broad specificity that prevents sharp discrimination of one type of anion channel from the others. Since the molecular entities of most anion channels or their core components have been identified, accumulating pieces of information on the basis of molecular understanding have progressively been added to anion channel pharmacology.

\section{A. ClC-2-Type Chloride Channel Blockers}

A group 12 heavy metal ion, $\mathrm{Cd}^{2+}$, which is a common air pollutant and a major component of cigarette smoke, is the most effective and well established blocker of ClC2 (for transfected ClC-2, see Schwiebert et al., 1998; Zúñiga et al., 2004; Cuppoletti et al., 2013; for endogenous ClC-2, see Chesnoy-Marchais and Fritsch, 1994; Bond et al., 1998; Clark et al., 1998; Enz et al., 1999; Blaisdell et al., 2000; Duan et al., 2000) with an $\mathrm{IC}_{50}$ of $48 \mu \mathrm{M}$ (Zúñiga et al., 2004) (see Table 2). $\mathrm{Cd}^{2+}$ is also known to block ClC-1 channel activity (Fahlke et al., 1998) and $\mathrm{Cl}^{-}$channel currents mediated by ClC-3a and ClC-3d overexpressed in the plasma membrane (Okada et al., 2014). $\mathrm{Cd}^{2+}$ is ineffective for VSOR (Fritsch and Edelman, 1997; Bond et al., 1998; Kajita et al., 2000;
Parkerson and Sontheimer, 2004) and CFTR (Wang et al., 2009; Infield et al., 2016) as well as for Maxi-Cl (see Fig. 12A) and ASOR (see Fig. 12B), whereas $\mathrm{Cd}^{2+}$ sensitivity of $\mathrm{CaCC}$ has not been studied thus far. In addition, it must be noted that voltage-gated $\mathrm{Ca}^{2+}$ channels are also blocked by $\mathrm{Cd}^{2+}$ (Hagiwara and Byerly, 1981; Lansman et al., 1986). $\mathrm{Cd}^{2+}$ does not affect activation but greatly accelerates the deactivation gate by binding to cysteine residue C256 in ClC-2 (Zúñiga et al., 2004). Another group 12 metal, $\mathrm{Zn}^{2+}$, blocks ClC-2 (Clark et al., 1998; Schwiebert et al., 1998; Huber et al., 2004) with an $\mathrm{IC}_{50}$ of $23 \mu \mathrm{M}$ (Clark et al., 1998). However, it is noted that $\mathrm{Zn}^{2+}$ blocks not only ClC-2 but also ClC-1 (Kürz et al., 1997, 1999) and Maxi-Cl (Schlichter et al., 1990; Kokubun et al., 1991; O'Donnell et al., 2001).

A carboxylate analog, 5-nitro-2-(3-phenylpropylamino)benzoic acid (NPPB), partially and voltage-dependently blocks ClC-2 (Clark et al., 1998; Furukawa et al., 1998; Xiong et al., 1999; Thompson et al., 2005) with a low affinity $\left(\mathrm{IC}_{50}=0.98 \mathrm{mM}\right.$; Furukawa et al., 1998). However, its specificity with inhibiting VSOR, Maxi$\mathrm{Cl}$, CFTR, and CaCC is very low, although NPPB was totally ineffective in blocking ASOR (Sato-Numata et al., 2016). ClC-2 is partially sensitive to other carboxylate analogs, anthracene-9-carboxylic acid (9-AC) and diphenylamine-2-carboxylate (DPC) (Thiemann et al., 1992; Clark et al., 1998; Schwiebert et al., 1998).

A scorpion venom peptide toxin, GaTx2, is a highaffinity ClC-2 blocker $\left(\mathrm{IC}_{50}=20 \mathrm{pM}\right)$ that is selective over other ClC family members (ClC-0, ClC- 1 , ClC-3, and ClC-4), CFTR, CaCC, and the $\mathrm{GABA}_{\mathrm{C}}$ receptor (Thompson et al., 2009). GaTx2 inhibits ClC-2 in a manner independent of voltage by slowing the activation gate without affecting deactivation and open channel conductance (Thompson et al., 2009). A synthetic opiate, methadone, was reported to voltageindependently block ClC-2 with an $\mathrm{IC}_{50}$ of $100 \mathrm{nM}$

TABLE 2

Pharmacological distinctions among VAACs and VCACs

\begin{tabular}{|c|c|c|c|c|c|c|}
\hline Chemical & ClC-2 & VSOR & Maxi-Cl & CFTR & $\mathrm{CaCC}$ & ASOR \\
\hline $\mathrm{Cd}^{2+}$ & + & - & - & - & NS & - \\
\hline $\mathrm{Gd}^{3+}$ & - & $-/+^{a}$ & $+\sim+++$ & - & - & - \\
\hline DCPIB & - & ++ & - & - & - & - \\
\hline DIDS (out) & - & $\pm \sim++\mathrm{VD}$ & + & - & $\pm \sim+\mathrm{VD}$ & $++\sim+++$ \\
\hline NPPB & $\pm \mathrm{VD}$ & + & + & $+++\mathrm{VD}$ & + & - \\
\hline NFA & - & \pm & - & $\pm \mathrm{VD}$ & $+\sim++$ & + \\
\hline Glibenclamide & NS & $\pm\left(\mathrm{VD}^{b}\right)$ & - & $+\sim++\mathrm{VD}$ & + & \pm \\
\hline Phloretin & NS & + & - & $\pm \mathrm{VD}$ & - & + \\
\hline CFTR $_{\text {inh }}-172$ & $+++\mathrm{VD}$ & $-/+\sim++^{a}$ & NS & +++ & - & NS \\
\hline $\mathrm{CaCC}_{\text {inh-A01 }}$ & NS & - & NS & NS & ++ & NS \\
\hline $\mathrm{T} 16 \mathrm{~A}_{\mathrm{inh}}-\mathrm{A} 01$ & NS & $-/++^{a}$ & NS & NS & $++\sim+++$ & NS \\
\hline GaTx1 & - & NS & NS & +++ & - & NS \\
\hline GaTx2 & +++ & NS & NS & - & - & NS \\
\hline Methadone & +++ & NS & NS & - & NS & NS \\
\hline
\end{tabular}

Insensitivity and sensitivity are denoted by minus and plus signs, respectively, as follows: -, insensitive; \pm , sensitive at sub-millimolar $\left(100 \leq \mathrm{IC}_{50}<1000 \mu \mathrm{M}\right)$ concentrations; + , sensitive at deca-micromolar $\left(10 \leq \mathrm{IC}_{50}<100 \mu \mathrm{M}\right)$ concentrations; ++, sensitive at micromolar $\left(1 \leq \mathrm{IC}_{50}<10 \mu \mathrm{M}\right)$ concentrations; and +++ , sensitive at sub-micromolar $\left(\mathrm{IC}_{50}<1 \mu \mathrm{M}\right)$ concentrations. NS, not studied; VD, voltage-dependent block.

${ }^{a}$ Depending on cell type (see the text for details).

${ }^{b}$ Voltage-dependent block only by the charged form. 
(Cuppoletti et al., 2013). The blockage mechanism is unclear but is different from the $\mathrm{Cd}^{2+}$ action and is not mediated by opioid receptors (Cuppoletti et al., 2013). Methadone was ineffective for CFTR (Cuppoletti et al., 2013), whereas its effects on other types of anion channels have not been studied. A thiazolidinone, CFTR inh -172 , was found to block not only CFTR (Ma et al., 2002; Kopeikin et al., 2010; Cuppoletti et al., 2014) but also ClC-2 with an $\mathrm{IC}_{50}$ of $143 \mathrm{nM}$ in a voltagedependent manner (Cuppoletti et al., 2014). In contrast, ClC-2 was not sensitive to stilbene-derivative anion channel blockers, 4-acetamido-4'-isothiocyano-2,2'disulfonic stilbene (SITS) and 4,4'-diisothiocyano-2,2' stilbenedisulfonic acid (DIDS) (Thiemann et al., 1992; Furukawa et al., 1998; Schwiebert et al., 1998; Xiong et al., 1999; Duan et al., 2000; Thompson et al., 2005), a DPC derivative niflumic acid (NFA) (Clark et al., 1998), tamoxifen (Duan et al., 2000), 4-[(2-butyl-6,7-dichloro-2cyclopentyl-2,3-dihydro-1-oxo-1H-inden-5-yl)oxy]butanoic acid (DCPIB) (Decher et al., 2001), and $\mathrm{Gd}^{3+}$ (ChesnoyMarchais and Fritsch, 1994; Tokimasa and North, 1996).

\section{B. Volume-Sensitive Outwardly Rectifying Anion Channel Blockers}

The most effective and selective VSOR blocker is DCPIB, which is an ethacrynic acid derivative, indanone compound (Decher et al., 2001). Its $\mathrm{IC}_{50}$ values were reported to be $3.5-4.1 \mu \mathrm{M}$ in calf bovine pulmonary artery endothelial cells (Decher et al., 2001), $2.3 \mu \mathrm{M}$ in mouse astrocytes (Liu et al., 2009), and approximately $3.5 \mu \mathrm{M}$ in human bronchial epithelial cells (Stott et al., 2014) (see Table 2). DCPIB blocks VSOR in a voltageindependent and fully reversible manner (Decher et al., 2001). This compound was shown to be ineffective in blocking any other anion channels, including ClC-1/2/ 4/5/K1, CaCC, and CFTR (Decher et al., 2001) as well as Maxi-Cl (Sabirov and Okada, 2005; Sabirov et al., 2016) and ASOR (Sato-Numata et al., 2016). However, DCPIB can also block some $\mathrm{K}^{+}$channels (Deng et al., 2016), connexin 43 hemichannels (Ye et al., 2009), and gastric proton pump $\mathrm{H}^{+}, \mathrm{K}^{+}$-ATPase (Fujii et al., 2015). Interestingly, a KCC antagonist, $R-(+)-[(2-n$-butyl-6,7dichloro-2-cyclopentyl-2,3-dihydro-1-oxo- $1 H$-inden-5-yl)oxy] acetic acid (DIOA) inhibits VSOR in rat thymocytes (Kurbannazarova et al., 2011) and blocks VSOR in HeLa cells with an $\mathrm{IC}_{50}$ of $20.9 \mu \mathrm{M}$ (Sato-Numata et al., 2016). DIOA is not as specific as DCPIB and also inhibits ASOR with approximately 10 times higher efficiency (SatoNumata et al., 2016). Structurally, both compounds are similar to a nonspecific $\mathrm{Cl}^{-}$channel blocker, indanyloxyacetic acid 94, and contain a 6,7-dichloro-2-cyclopentyl-1oxo-indanyl core with an oxy-carbonic acid (oxyacetate in DIOA and indanyloxyacetic acid 94 , and oxybutyrate in DCPIB) connected to oxo-indanyl at position 5 (Fig. 9A). One may suggest that a short-chain oxy-carbonic acid combined with a chlorinated cyclopentyl-oxo-indanyl fragment represents a structural determinant for VSOR block. We note that both DCPIB and DIOA also contain an $n$-butyl radical connected to the oxo-indanyl at position 2 (the same place as cyclopentyl); therefore, the only difference between DCPIB and DIOA is the length of the oxy-carbonic acid, which is slightly longer (by $-\mathrm{CH}_{2}-\mathrm{CH}_{2}$ ) in DCPIB (Fig. 9A). This slight difference may provide greater specificity for DCPIB over DIOA.

A bisphenol, phloretin, also blocks VSOR, in a voltage-independent and fully reversible manner, only from the extracellular side with an $\mathrm{IC}_{50}$ of approximately $30 \mu \mathrm{M}$, without affecting time-dependent inactivation kinetics at large positive potentials (Fan et al., 2001). Similar to DIDS and SITS, phloretin contains two aromatic benzene rings but lacks the negative charge (Fig. 9B), which may explain the voltage-independent blocking. Phloridzin, which is a 2 '- $\beta$-D-glucopyranoside-derivative of phloretin, was absolutely ineffective, suggesting that the hydroxyl group at the ortho-position of the carbonyl fragment as well as the overall shape and presumably hydrophobicity are important structural determinants for exerting channel inhibition (Fan et al., 2001). CaCC was insensitive to phloretin, whereas CFTR was sensitive to this compound at higher concentrations $\left(\mathrm{IC}_{50}=195\right.$ and $355 \mu \mathrm{M}$ at -60 and $+60 \mathrm{mV}$, respectively) (Fan et al., 2001). Maxi-Cl is insensitive to phloretin (Sabirov et al., 2001; Dutta et al., 2002; Liu et al., 2006, 2008b). However, ASOR was voltage-independently sensitive to phloretin with an $\mathrm{IC}_{50}$ of $17.5 \mu \mathrm{M}$ (Wang et al., 2007). An acidic di-aryl-urea, $N$-[3,5-bis(trifluoromethyl)-phenyl]- $N$-[4bromo-2-(1H-tetrazol-5-yl)-phenyl] urea (NS3728), was reported to block VSOR with high affinity $\left(\mathrm{IC}_{50}=0.30\right.$ $0.46 \mu \mathrm{M}$; Hélix et al., 2003; Klausen et al., 2007) in a manner independent of voltage (Hélix et al., 2003). However, CaCC was also very sensitive to NS3728 (Klausen et al., 2007) with an $\mathrm{IC}_{50}$ of $1.3 \mu \mathrm{M}$ (Sauter et al., 2015). Since the sensitivity of ClC-2, Maxi-Cl, CFTR, and ASOR to NS3728 has not yet been examined, the specificity of this compound remains questionable.

VSOR is known to be sensitive to a number of conventional $\mathrm{Cl}^{-}$channel blockers (see Okada, 1997). The stilbene-derivative blockers, SITS and DIDS, can inhibit VSOR in a voltage-dependent manner (Kubo and Okada, 1992; Nilius et al., 1994a; Shen et al., 1996). The reported $\mathrm{IC}_{50}$ values for SITS at positive and negative voltages are 1.5 and $6 \mu \mathrm{M}$, respectively, in Intestine 407 cells (Kubo and Okada, 1992), whereas those for DIDS are 22.7 and $88.5 \mu \mathrm{M}$ in HeLa cells (Fig. 10 ), 0.84 and $11 \mu \mathrm{M}$ in canine colonic myocytes (Dick et al., 1999), 26 and $256 \mu \mathrm{M}$ in HEK293 cells (Hélix et al., 2003), 19.8 and $97.4 \mu \mathrm{M}$ in normal human bronchial epithelial cells, and 9.0 and $81.4 \mu \mathrm{M}$ in human cystic fibrosis bronchial epithelial cells (Stott et al., 2014), respectively. Such voltage-dependent blocking effects may be caused by open-channel blocking, because the size of divalent anion SITS (effective radius approximately $0.55 \mathrm{~nm}$; Okada et al., 2009b) and DIDS 

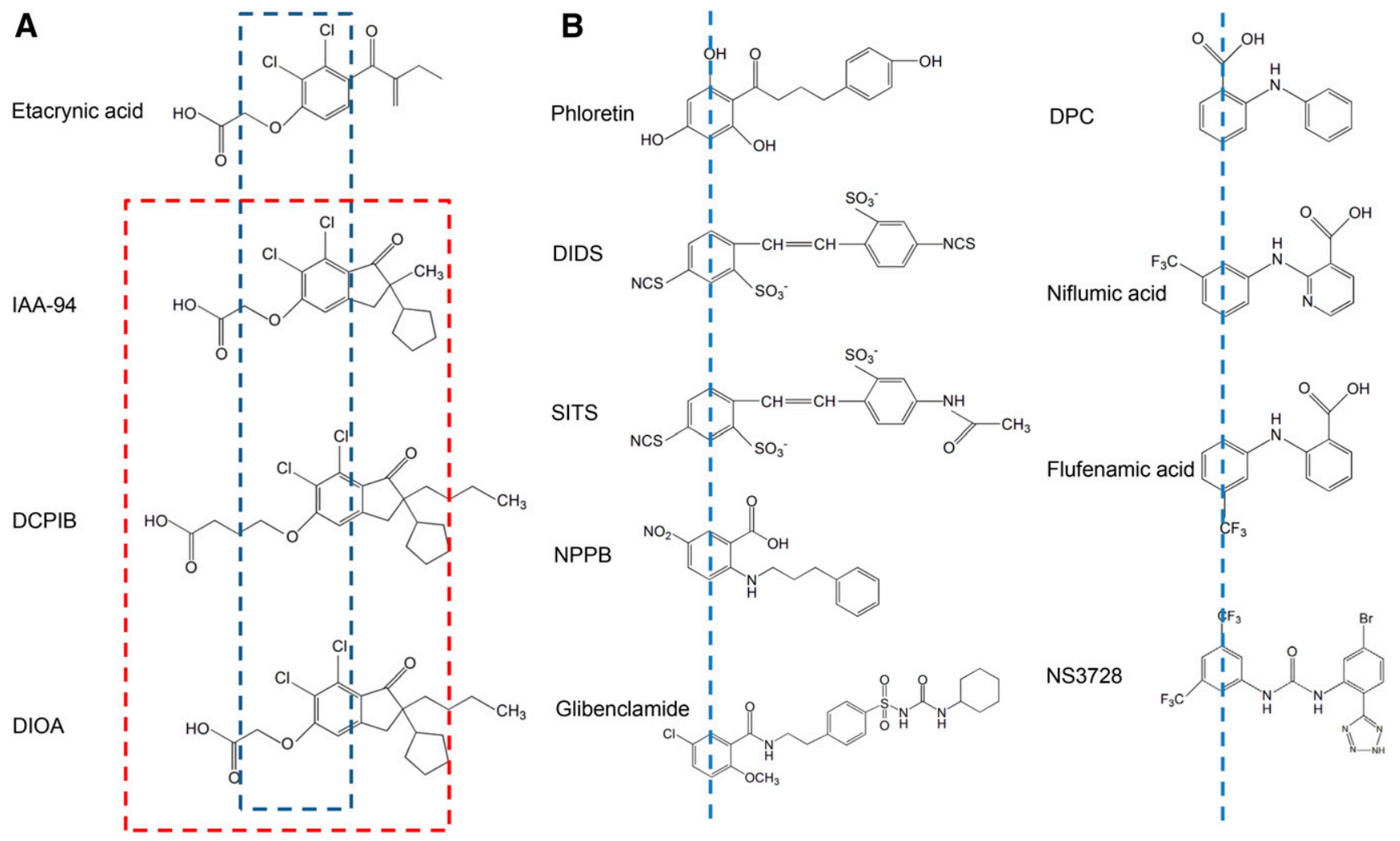

Fig. 9. Chemical structures of some VSOR blockers. (A) Etacrynic acid derivatives. The blue dashed line depicts the 2,3-dichlorophenoxy fragment, which is identical for all of these molecules. The red dashed line delineates a fragment (short-chain oxy-carbonic acid combined with chlorinated cyclopentyl-oxo-indanyl) common for VSOR blockers (see the text for details) but not for etacrynic acid. (B) VSOR blockers that have two aromatic rings connected with a chain of one to four atoms (carbon, nitrogen, or mixed). The blue dashed lines mark the first aromatic ring (see the text for details). IAA-94, indanyloxyacetic acid 94 .

(approximately $0.54 \mathrm{~nm}$; Okada et al., 2009b) is close to the cut-off radius of the VSOR pore (approximately $0.63 \mathrm{~nm}$; Ternovsky et al., 2004). Although DIDS can inhibit Maxi-Cl and ASOR as well (see below), this inhibition was not dependent on voltages. Open-channel blocking of VSOR is also induced by extracellular, but not intracellular, application of negatively charged ATP with an $\mathrm{IC}_{50}$ of $2-5 \mathrm{mM}$ at positive potentials (Nilius et al., 1994b; Tsumura et al., 1996), suggesting that ATP enters the pore only from its extracellular end to reach the ATP binding site inside the channel pore. A sulfonylurea, glibenclamide, exerts blocking actions on VSOR at positive voltages from the extracellular (not intracellular) side at physiologic $\mathrm{pH}$ by mixed mechanisms produced by charged and uncharged forms $\left(\mathrm{IC}_{50}=232 \mu \mathrm{M}\right.$ at $\left.\mathrm{pH} 7.5\right)$ : its uncharged form voltage-independently inhibits VSOR with an $\mathrm{IC}_{50}$ of $2.8 \mu \mathrm{M}$ at $\mathrm{pH} 5$, whereas the negatively charged form voltage-dependently inhibits VSOR with an $\mathrm{IC}_{50}$ of $532 \mu \mathrm{M}$ at $\mathrm{pH} 9.2$ (Liu et al., 1998). It must be noted that the effective radii of $\mathrm{ATP}^{4-}$ (approximately $0.58 \mathrm{~nm}$ ) and glibenclamide ${ }^{-}$(approximately $0.6 \mathrm{~nm}$ ) are close to the VSOR pore size (Okada et al., 2009b). A hexavalent anion, suramin, also exhibits a similar voltage-dependent blocking action for VSOR currents with $\mathrm{IC}_{50}$ values of $1.5 \mu \mathrm{M}$ in nasopharyngeal CNE-2Z cells (Yang et al., 2015) and 8.3 $\mu \mathrm{M}$ in HeLa cells (SatoNumata et al., 2016) at positive voltages. The effective radius of a minor axis (approximately $0.7 \mathrm{~nm}$ ) of ovalshaped suramin is again close to the VSOR pore size (Okada et al., 2009b).

Carboxylate analogs [NPPB, DPC, and 9-AC] as well as DPC derivatives [NFA and flufenamic acid (FFA)] are also classified into conventional $\mathrm{Cl}^{-}$channel blockers and inhibit VSOR in a voltage-independent manner (Okada, 1997). The $\mathrm{IC}_{50}$ values reported for NPPB are $25 \mu \mathrm{M}$ in Intestine 407 cells (Kubo and Okada, 1992), 35-37 $\mu \mathrm{M}$ in rat inner medullary collecting duct cells (Boese et al., 1996), $21 \mu \mathrm{M}$ in HEK293 cells (Hélix et al., 2003), $30.2 \mu \mathrm{M}$ in human bronchial epithelial cells (Stott et al., 2014), 36.6 $\mu \mathrm{M}$ in cystic fibrosis bronchial epithelial cells (Stott et al., 2014), and $68 \mu \mathrm{M}$ in astrocytoma cells (Bakhramov et al., 1995), whereas those for DPC and NFA are $350 \mu \mathrm{M}$ (Kubo and Okada, 1992) and $320 \mu \mathrm{M}$ (Sato-Numata et al., 2016), respectively.

In comparing the structures of phloretin, DIDS, SITS, DPC, NFA, FFA, NPPB, NS3728, and glibenclamide, one may notice that all of them have two aromatic rings connected with a chain of 1-4 atoms (carbon, nitrogen, or mixed) (Fig. 9B). This motif may represent another important structural determinant of VSOR blockers.

VSOR activity was sensitive, in a manner independent of membrane potentials, to a large variety of other structurally unrelated drugs, including a 
A
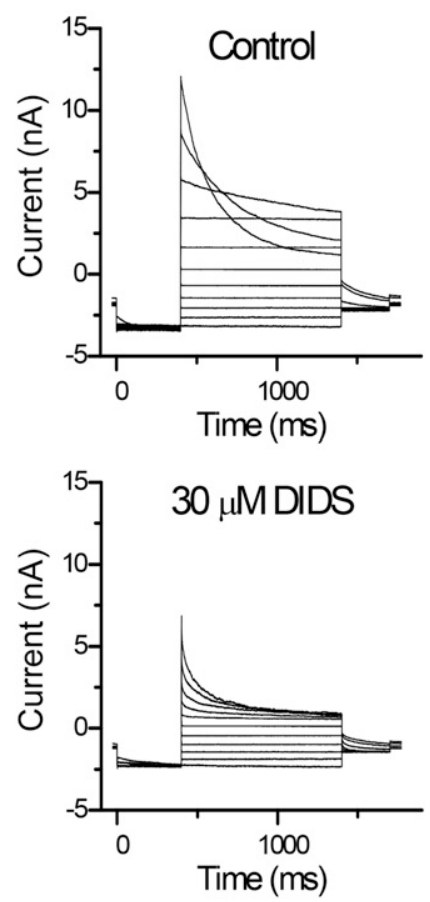
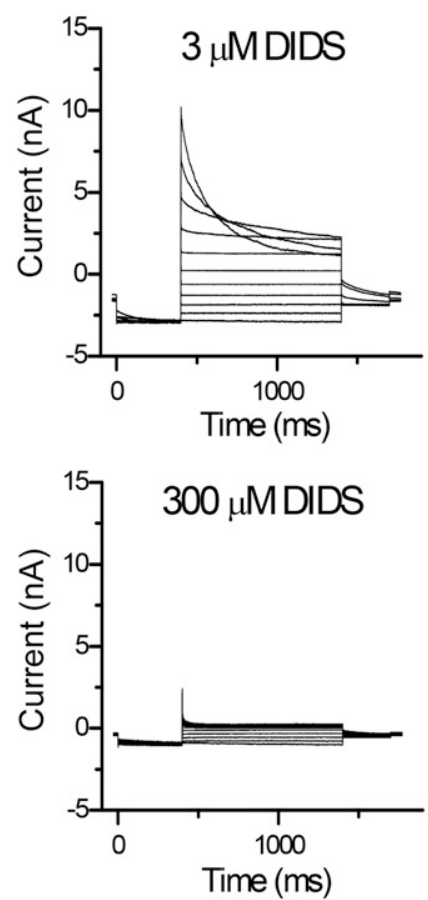

B

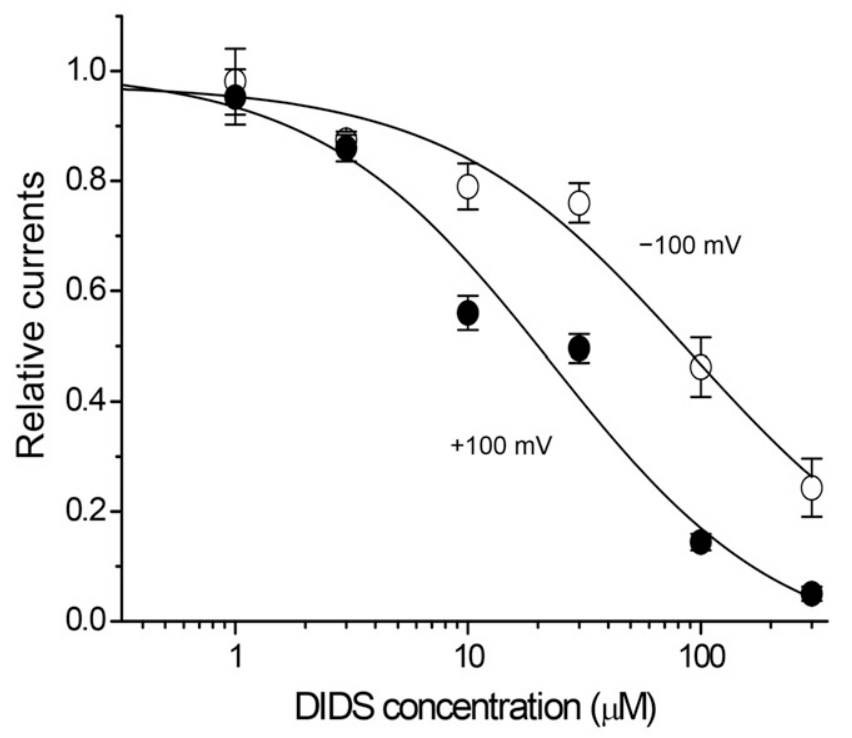

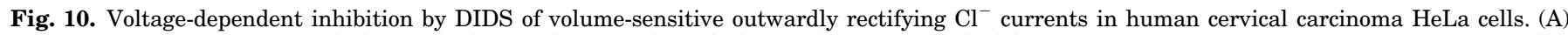

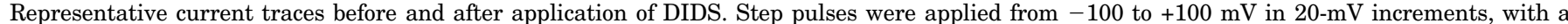

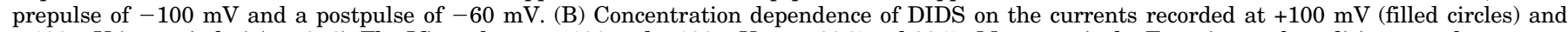

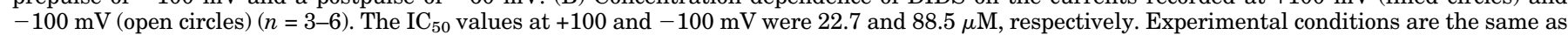
reported previously (Shimizu et al., 2004).

polyunsaturated fatty acid, arachidonic acid $\left(\mathrm{IC}_{50}=\right.$ $8 \mu \mathrm{M}$; Kubo and Okada, 1992); oxonol dyes, bis-(1,3dibutylbarbituric acid)pentamethine oxanol (diBA-(5)$\left.\mathrm{C}_{4}\right)\left(\mathrm{IC}_{50}=1.8 \mu \mathrm{M}\right.$; Arreola et al., 1995) and WW781 [4-[4-[(1E,3E)-5-(1,3-dibutyl-2,4,6-trioxo-1,3-diazinan5-ylidene)penta-1,3-dienyl]-3-methyl-5-oxo-4H-pyrazol-1yl]benzenesulfonic acid] $\left(\mathrm{IC}_{50}=25 \mu \mathrm{M}\right.$; Behe et al., 2017); an antimalaria drug, mefloquine $\left(\mathrm{IC}_{50}=1.2\right.$ $\mu \mathrm{M}$; Maertens et al., 2000); a hemichannel blocker, carbenoxolone $(\mathrm{CBX})\left(\mathrm{IC}_{50}=15.4 \mu \mathrm{M}\right.$; Benfenati et al., 2009); and cell-permeable CFTR inhibitors, GlyH-101 $\left(\mathrm{IC}_{50}=5\right.$ to $6 \mu \mathrm{M}$; Melis et al., 2014$)\left(\mathrm{IC}_{50}=9.5 \mu \mathrm{M}\right.$; Friard et al., 2017) and PPQ-102 [6,7-dihydro-7,9-dimethyl-6-(5-methyl-2-furanyl)-11-phenylpyrimido $\left[4^{\prime}, 5^{\prime}\right.$ : $3,4]$ pyrrolo[1,2- $a$ ]quinoxaline-8,10(5H,9)-dione] $\left(\mathrm{IC}_{50}=\right.$ $19.6 \mu \mathrm{M}$; Friard et al., 2017). Inhibition of VSOR was also reported to be induced by a purinergic $\mathrm{P} 2 \mathrm{X}$ receptor blocker, pyridoxalphosphate-6-azophenyl-2', $4^{\prime}$-disulfonic acid (Galietta et al., 1997; Darby et al., 2003; Poletto Chaves and Varanda, 2008), in a voltage-dependent manner and by unprotonated quinidine in a voltageindependent manner (at pH 9; Voets et al., 1996; Behe et al., 2017). Since the drugs mentioned in this paragraph have no obvious common structural motives, we assume that VSOR channel protein has several pockets (binding sites) of different shapes and electrostatics to accommodate chemical substances of very different structures. It is also possible that some of these drugs affect the regulatory auxiliary proteins but not the channel itself.

The VSOR currents were found to be voltagedependently inhibited by a tyrosine kinase inhibitor, genistein (Bryan-Sisneros et al., 2000; Shuba et al., 2000; Inoue et al., 2007), but not by its inactive analog daidzein (Inoue et al., 2007). An antiestrogen, tamoxifen, which is a known inhibitor of P-glycoprotein, was found to voltage-independently inhibit VSOR activity in a number of nonexcitable cells (Zhang et al., 1994; Tominaga et al., 1995b; Meyer and Korbmacher, 1996; Wondergem et al., 2001; Chen et al., 2002; Hélix et al., 2003; Yang et al., 2015) and colonic smooth muscle cells (Dick et al., 1999). In contrast, tamoxifen was ineffective in inhibiting VSOR in neuronal (Leaney et al., 1997; Inoue et al., 2005, 2007; Zhang et al., 2011) and muscular (Voets et al., 1997) cells as well as in some epithelial cells (Winpenny et al., 1996; Mitchell et al., 1997b) and neutrophils (Ahluwalia, 2008b). In contrast, it is noted that tamoxifen blocks some cationic channels in neuronal cells (Allen et al., 1998; Hardy et al., 1998) and muscular cells (He et al., 2003) as well as voltage-gated $\mathrm{Ca}^{2+}$ and $\mathrm{K}^{+}$channels in canine colonic myocytes at $10 \mu \mathrm{M}$ (Dick et al., 1999). Since tamoxifen was found to inhibit NOX activity in human neutrophils (Ahluwalia, 2008b), there is a possibility that tamoxifen sensitivity of VSOR activities in some cell types is an indirect effect mediated by NOX inhibition (see Fig. 4; 
type B mechanism). Other P-glycoprotein inhibitors, such as verapamil, nifedipine, 1,9-dideocyforskolin, and quinidine, were reported to inhibit VSOR in some, but not all, cell types (see Okada, 1997),

VSOR activity was insensitive to heavy metal $\mathrm{Cd}^{2+}$ and $\mathrm{Zn}^{2+}$ (Okada et al., 2009b). $\mathrm{Gd}^{3+}$ was also ineffective at $30 \mu \mathrm{M}$ for VSOR activity in many nonexcitable cells (Hazama et al., 1999, 2000; Sabirov et al., 2001; Liu et al., 2006) but sizably suppressed VSOR currents in canine colonic myocytes at $100 \mu \mathrm{M}\left(\mathrm{IC}_{50}=23 \mu \mathrm{M}\right.$; Dick et al., 1999). VSOR currents were largely insensitive to $\mathrm{CaCC}$ inhibitors,

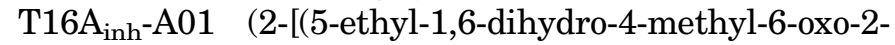
pyrimidinyl)thio]- $N$-[4-(4-methoxyphenyl)-2-thiazolyl]acetamide) and $\mathrm{CaCC}_{\text {inh }}$-A01 [6-(1,1-dimethylethyl)-2[(2-furanylcarbonyl)amino]-4,5,6,7-tetrahydrobenzo[b]thiophene-3-carboxylic acid] in pancreatic ductal adenocarcinoma cells (Sauter et al., 2015), but they were recently reported to be sensitive to $\mathrm{T} 16 \mathrm{~A}_{\mathrm{inh}}-\mathrm{A} 01$ with an $\mathrm{IC}_{50}$ of $5.5 \mu \mathrm{M}$ in HEK293 cells (Friard et al., 2017). CFTR $_{\text {inh }}-172$ was found to be ineffective in inhibiting VSOR in FRT cells (Ma et al., 2002) and in HEK293 cells (Friard et al., 2017) but was reported to voltageindependently inhibit VSOR in mouse kidney and hamster PS120 cells with $\mathrm{IC}_{50}$ values of 12 and $5.3 \mu \mathrm{M}$, respectively (Melis et al., 2014).

\section{Maxi-Anion Channel Blockers}

Heavy metal ion $\mathrm{Gd}^{3+}$ is the most effective blocker for Maxi-Cl (Sabirov et al., 2001, 2006; Bell et al., 2003; Dutta et al., 2004, 2008; Liu et al., 2006, 2008a; Toychiev et al., 2009; Kurbannazarova et al., 2011; Islam et al., 2012; Sabirov et al., 2017) with $\mathrm{IC}_{50}$ values of $0.75 \mu \mathrm{M}$ in $\mathrm{C} 127$ cells (unpublished data) and $46 \mu \mathrm{M}$ in L929 cells (Islam et al., 2012) (see Table 2). In addition, the blocking action was observed from outside (Fig. 11A), but not inside, the membrane (Sabirov et al., 2001; Toychiev et al., 2009) in a manner independent of voltage (Sabirov et al., 2001; Dutta et al., 2004). Gd ${ }^{3+}$ is ineffective in blocking other anion channels, including ClC-2 (Chesnoy-Marchais and Fritsch, 1994; Tokimasa and North, 1996), VSOR (Hazama et al., 1999, 2000; Sabirov et al., 2001; Liu et al., 2006; Okada et al., 2009b), CFTR (Hazama et al., 2000), CaCC (Waniishi et al., 1998), and ASOR (Sato-Numata et al., 2016), in many cell types. In contrast, however, $\mathrm{Gd}^{3+}$ was found to block a variety of nonanionic channels, including voltage-dependent $\mathrm{Ca}^{2+}$ channels (Docherty, 1988; Biagi and Enyeart, 1990; Dick et al., 1999), delayed $\mathrm{K}^{+}$ channels (Dick et al., 1999), stretch-activated cation channels (Gustin et al., 1986; Yang and Sachs, 1989), Piezo1 channels (Coste et al., 2010), a number of transient receptor potential channel members (Zitt et al., 1996; Strotmann et al., 2000; Grimm et al., 2003; Hermosura et al., 2002; Hanano et al., 2004), and acid-sensing ion channels (Babinski et al., 2000). Another metal ion, $\mathrm{Zn}^{2+}$, is also an effective blocker of Maxi-Cl (Schlichter et al., 1990; Kokubun et al., 1991;
Pahapill and Schlichter, 1992; O’Donnell et al., 2001). The detailed mechanisms for blocking of Maxi-Cl by $\mathrm{Gd}^{3+}$ and $\mathrm{Zn}^{2+}$ remain unclear.

Another effective blocker of $\mathrm{Maxi}-\mathrm{Cl}$ is arachidonic acid (Riquelme and Parra, 1999; Dutta et al., 2002, 2004, 2008; Liu et al., 2006, 2008a; Sabirov et al., 2006, 2016; Islam et al., 2012) (Fig. 11A) with an $\mathrm{IC}_{50}$ of 4 to $5 \mu \mathrm{M}$ (Dutta et al., 2002). However, arachidonic acid is also known to inhibit other anion channels such as VSOR (Kubo and Okada, 1992; Sato-Numata et al., 2016), CFTR (Linsdell, 2000; Zhou and Linsdell, 2007), and ASOR (Sato-Numata et al., 2016) with $\mathrm{IC}_{50}$ values of 3.5-50.5 $\mu \mathrm{M}$. Maxi-Cl is inhibited by arachidonic acid itself (not by the downstream metabolic products) by two different mechanisms: one is channel shutdown with an $\mathrm{IC}_{50}$ of 4 to $5 \mu \mathrm{M}$, and the other is a unitary conductance reduction with an $\mathrm{IC}_{50}$ of 13 to $14 \mu \mathrm{M}$ without affecting voltage-dependent changes in the open probability of Maxi-Cl. A negative charge and specific cis-conformation of the fatty acid chain are structural determinants important for blockage, which occurred only from the cytosolic side (Dutta et al., 2002). We recently identified SLCO2A1, known thus far as $\mathrm{PGT}$, as the core component of Maxi-Cl and we found that it was very sensitive to PGT blocker BSP $\left(\mathrm{IC}_{50}=\right.$ 6.8-7.8 $\mu \mathrm{M}$ ) as well as to other PGT blockers bromocresol green and indocyanine green (Sabirov et al., 2017). BSP was effective from both sides of the membrane, producing a reversible, dose-dependent flickery blockage in a voltage-independent manner, which rules out the pore plug-in open-channel block mechanism.

Conventional anion channel blockers NPPB, SITS, and DIDS are also effective for Maxi-Cl, but their blocking effects are only partial (see reviews by Sabirov and Okada, 2004a, 2005, 2009; Sabirov et al., 2016). The Maxi-Cl current was voltage-independently blocked by NPPB with an $\mathrm{IC}_{50}$ of approximately $19 \mu \mathrm{M}$ (Sun et al., 1992), as well as by SITS (Kokubun et al., 1991; Mitchell et al., 1997a; O'Donnell et al., 2001; Bell et al., 2003; Do et al., 2004; Liu et al., 2006; Islam et al., 2012) and by DIDS (Light et al., 1990; Sun et al., 1992; Brown et al., 1993; Riquelme and Parra, 1999) with an $\mathrm{IC}_{50}$ of approximately $100 \mu \mathrm{M}$ only from outside the cell membrane (Groschner and Kukovetz, 1992).

Maxi-Cl activity was not virtually affected by a ClC-2 blocker, $\mathrm{Cd}^{2+}(1 \mathrm{mM})$, as shown in Fig. 12A. Maxi-Cl currents were observed to be insensitive to a variety of other anion channel blockers, including DCPIB (Sabirov et al., 2016) (Fig. 11B), phloretin (Sabirov et al., 2001; Dutta et al., 2004; Liu et al., 2006), 9-AC (Kokubun et al., 1991; O'Donnell et al., 2001), NFA (Sabirov et al., 2001), and CBX (De Marchi et al., 2008; Sabirov et al., 2016) (Fig. 11D). Maxi-Cl is also insensitive to pannexin hemichannel antagonists, probenecid, mefloquine, and ${ }^{10} \mathrm{Panx} 1$, as well as to connexin hemichannel antagonists, 1-octanol and gap junction peptide 27, GAP27, in 

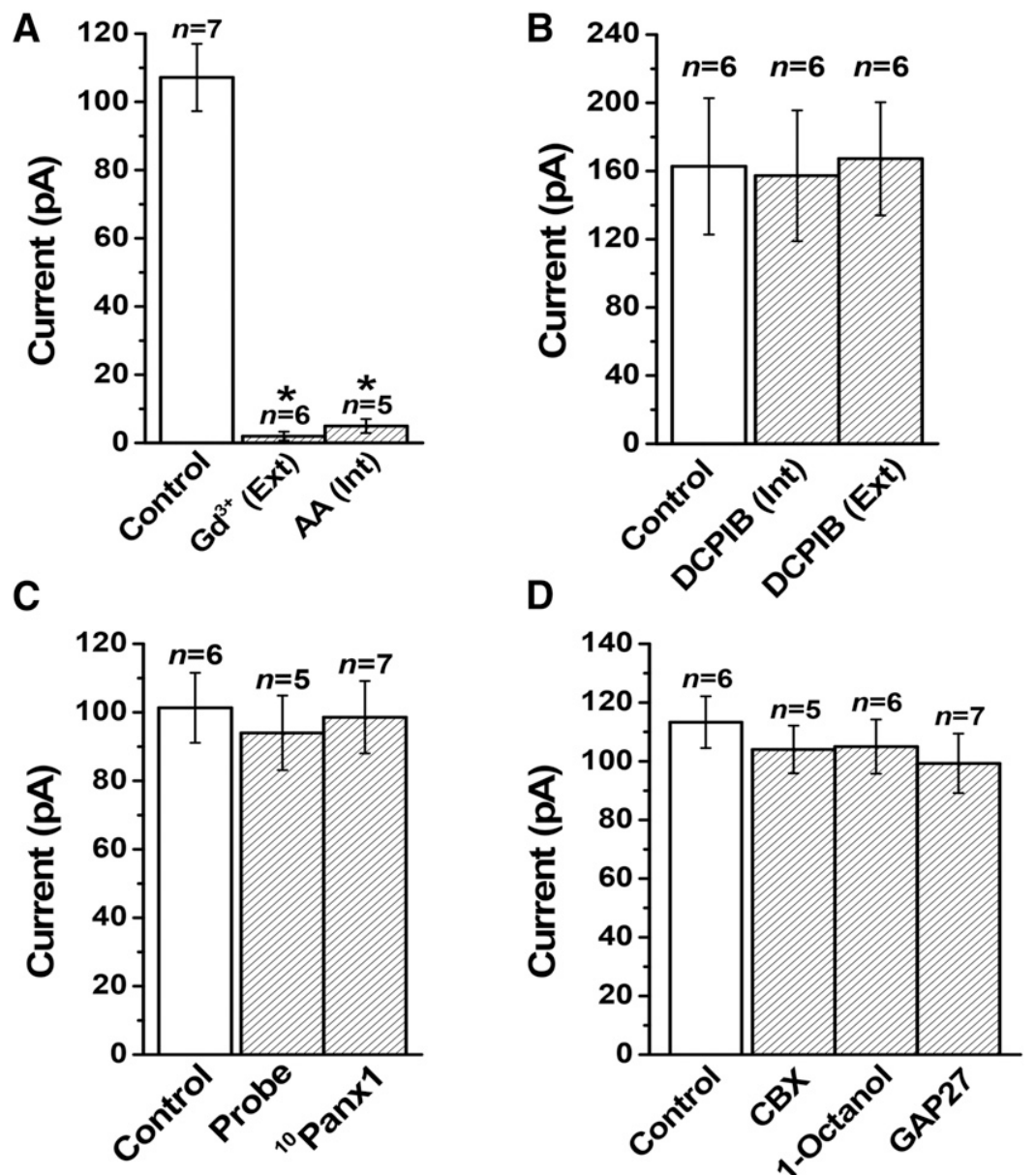

Fig. 11. Pharmacological profile of Maxi-Cl currents in patches excised from $\mathrm{C} 127$ cells. All of the columns represent the mean currents recorded at $+25 \mathrm{mV}$. (A) Inhibitory effects of extracellular application of $\mathrm{Gd}^{3+}(50 \mu \mathrm{M})$ and intracellular application of arachidonic acid (20 $\left.\mu \mathrm{M}\right)$. (B) Insensitivity to a VSOR blocker, DCPIB $(10 \mu \mathrm{M})$, added from the intracellular or extracellular side. (C) Insensitivity to pannexin hemichannel antagonists, probenecid $(1 \mathrm{mM})$ and ${ }^{10} \mathrm{Panx} 1(8 \mu \mathrm{g} / \mathrm{ml})$, added to the extracellular (pipette) solution. (D) Insensitivity to gap junction antagonists, CBX (100 $\mu \mathrm{M}$ ), 1-octanol $(2 \mathrm{mM})$, and GAP27 $(4 \mu \mathrm{g} / \mathrm{ml})$, added to the extracellular (pipette) solution. Experimental conditions, other than the cell line used, are the same as reported previously (Islam et al., 2012; Sabirov et al., 2017). AA, arachidonic acid; Probe, probenecid.

L929 cells (Islam et al., 2012) and in C127 cells (Fig. 11, $\mathrm{C}$ and D). DPC was found to be ineffective for Maxi-Cl currents in B lymphocytes (Bosma, 1989), the placenta (Brown et al., 1993), and kidney macula densa cells (Bell et al., 2003) but was effective for Maxi-Cl currents in kidney cortical collecting duct cells (Light et al., 1990).

\section{Cystic Fibrosis Transmembrane Conductance Regulator Blockers}

The sulfonylureas glibenclamide and tolbutamide are effective blockers not only of ATP-sensitive potassium channels but also of CFTR. These negatively charged substances exert actions as open-channel blockers on CFTR by reducing open channel probability (Schultz et al., 1996, 1997; Venglarik et al., 1996; Sheppard and Robinson, 1997), with $\mathrm{IC}_{50}$ values of $2-38 \mu \mathrm{M}$ for glibenclamide (Sheppard and Welsh, 1992; Rabe et al., 1995; Tominaga et al., 1995a; McNicholas et al., 1996; Schultz et al., 1996) and approximately $150 \mu \mathrm{M}$ for tolbutamide (Sheppard and Welsh, 1992). However, glibenclamide was also known to block VSOR (Yamazaki and Hume, 1997; Liu et al., 1998), CaCC (Yamazaki and Hume, 1997), and ASOR (Yamamoto and Ehara, 2006) at relatively higher concentrations. A diarylsulfonylurea, N(4-methylphenylsulfonyl)-N-(4-trifluoromethylphenyl)urea, DASU-02, is also an effective blocker for CFTR but not for ClC-2 (O'Donnell et al., 2000). A number of conventional anion channel blockers, including arylaminobenzoates (NPPB, DPC, and NFA), an aromatic carboxylic acid (9-AC), and disulfonic stilbenes (DIDS and DNDS), are also negatively charged and effective in blocking CFTR in a voltagedependent manner. Among them, NPPB is most effective, exhibiting an $\mathrm{IC}_{50}$ value of $80 \mathrm{nM}$ (Wangemann et al., 1986), although the blocking action of NPPB is nonspecific with blocking of all other VAACs and VCACs, except ASOR, at higher concentrations (see other parts of this section and Table 2). DPC and NFA can inhibit CFTR, although also nonspecifically and voltage-dependently. IC $_{50}$ values for DPC are $237 \mu \mathrm{M}$ at $+100 \mathrm{mV}$ and $912 \mu \mathrm{M}$ at $0 \mathrm{mV}$ (McCarty et al., 1993), whereas the $\mathrm{IC}_{50}$ for NFA 
A
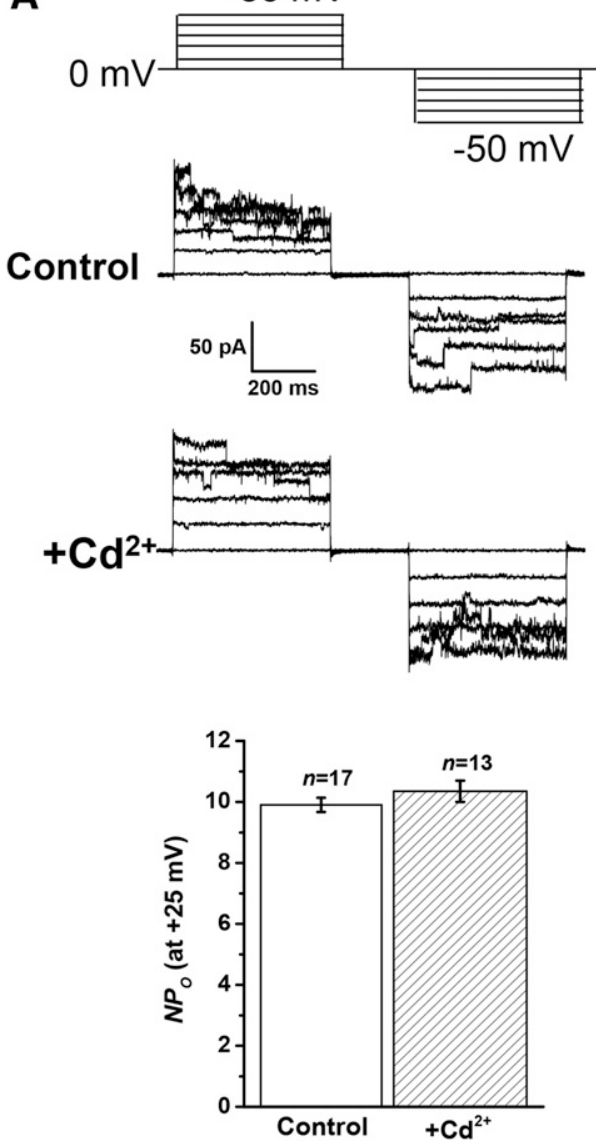

B

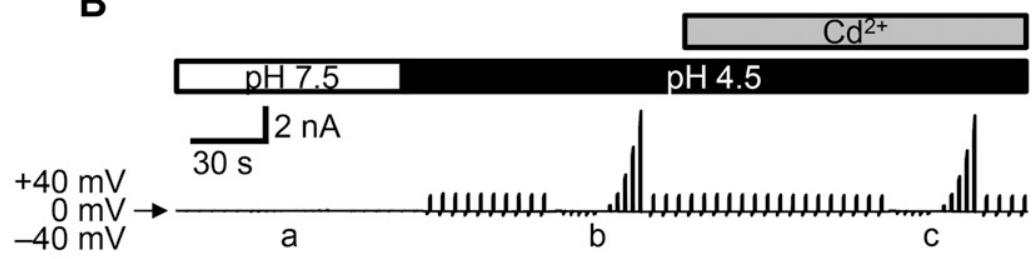

a

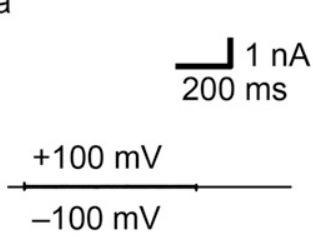

b

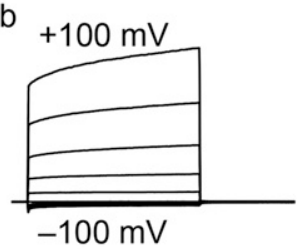

C

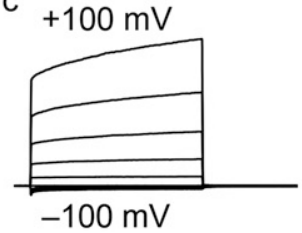

Fig. 12. $\mathrm{Cd}^{2+}$ insensitivity of Maxi-Cl (A) and ASOR (B) currents. (A) Representative Maxi-Cl currents recorded in inside-out patch membranes excised from $\mathrm{C} 127$ cells in the absence (control) and presence $\left(+\mathrm{Cd}^{2+}\right)$ of $1 \mathrm{mM} \mathrm{Cd}^{2+}$ added to the extracellular (pipette) solution during application of step pulses from 0 to $\pm 50 \mathrm{mV}$ in $10-\mathrm{mV}$ increments (protocol shown at the top). The bottom panel shows mean ( \pm S.E.M.) $N P_{\mathrm{o}}$ values measured at $+25 \mathrm{mV}$ in the absence and presence of $\mathrm{Cd}^{2+}$. There was no significant difference (at $P>0.05$ ) between the two values. Experimental conditions are the same as reported previously (Islam et al., 2012; Sabirov et al., 2017). (B) Whole-cell ASOR currents recorded in HeLa cells in the absence and presence of 1 mM $\mathrm{Cd}^{2+}$ added to the bath solution. The upper panel shows whole-cell currents before ( $\mathrm{pH} 7.5$ ) and during ( $\mathrm{pH} 4.5$ ) exposure to acidic bath solution in the absence and presence of $\mathrm{Cd}^{2+}$. The currents were elicited by application of alternating pulses from 0 to $\pm 40 \mathrm{mV}$ or of step pulses (at a, $\mathrm{b}$, and c) from -100 to $+100 \mathrm{mV}$ in $20-\mathrm{mV}$ increments. The middle panel shows expanded traces of current responses (at a, b, and c) to the step pulses. The bottom panels show $I$ - $V$ relationships for the whole-cell current densities at $\mathrm{pH} 7.5$ (squares) and $\mathrm{pH} 4.5$ in the absence (circles) and presence (triangles) of $\mathrm{Cd}^{2+}$. There was no significant difference (at $P>0.05$ ) between the circles and triangles at given voltages. Experimental conditions are the same as reported previously (Sato-Numata et al., 2016). $N P_{\mathrm{o}}$, number of open channels.

is $253 \mu \mathrm{M}$ at $-50 \mathrm{mV}$ (Scott-Ward et al., 2004). 9-AC was reported to block CFTR from the extracellular side of cell membranes with $\mathrm{IC}_{50}$ values of $942 \mu \mathrm{M}$ at $-100 \mathrm{mV}$ in guinea pig ventricular cells (Zhou et al., 1997) and 2.55, 1.70 , and $1.26 \mathrm{mM}$ at $-60,-80$ and $-100 \mathrm{mV}$, respectively, in CFTR-transfected 3T3 cells (Ai et al., 2004), whereas 9-AC rather exhibited an enhancing effect on cardiac CFTR activity from the intracellular side by inhibiting some protein phosphatases (Zhou et al., 1997). DIDS and DNDS inhibit CFTR only from the cytosolic side in a manner dependent on voltages with an $\mathrm{IC}_{50}$ of 236 and $80 \mu \mathrm{M}$, respectively, at $0 \mathrm{mV}$ (Linsdell and Hanrahan, 1996; also see Schultz et al., 1999). It is deemed that these negatively charged blockers enter the pore from its cytoplasmic end to reach the positively charged binding site, especially at lysine residue K95 in TM1, inside the channel pore (Linsdell, 2005, 2014; Zhou et al., 2010).

So-called more potent CFTR blockers have recently become available. A thiazolidinone, $\mathrm{CFTR}_{\mathrm{inh}}-172$, voltage-independently inhibits CFTR activity in FRT cells overexpressing CFTR with an $\mathrm{IC}_{50}$ of $300 \mathrm{nM}$ (Ma et al., 2002) and in HEK293 cells overexpressing CFTR with an $\mathrm{IC}_{50}$ of $133.6 \mathrm{nM}$ (Cuppoletti et al., 2014). In addition, $\mathrm{CFTR}_{\mathrm{inh}}-172$ was found to inhibit human, but not shark, CFTR activity in the oocyte expression system with an $\mathrm{IC}_{50}$ of $10.4 \mu \mathrm{M}$ (Stahl et al., 2012). Arginine 347 (R347), which does not contribute to CFTR pore formation, was identified to be a key position for the blocking action of CFTR $_{\text {inh }}-172$ (Caci et al., 2008; Zegarra-Moran and Galietta, 2017). However, it is noted that $\mathrm{CFTR}_{\text {inh }}-172$ was found to inhibit ClC-2 equipotently $\left(\mathrm{IC}_{50}=143 \mathrm{nM}\right)($ Cuppoletti et al., 2014) and also VSOR in some cell types (Melis et al., 2014) but not in other cell types (Ma et al., 2002; Friard et al., 2017). A glycine hydrazide, GlyH-101, voltage-dependently suppresses epithelial CFTR activity with $\mathrm{IC}_{50}$ values of 1.4 and $5.6 \mu \mathrm{M}$ at +60 and $-60 \mathrm{mV}$, respectively, from the extracellular side 
(Muanprasat et al., 2004) and cardiac CFTR activity with $\mathrm{IC}_{50}$ values of 0.3 and $5.1 \mu \mathrm{M}$ at +100 and $100 \mathrm{mV}$, respectively (Barman et al., 2011). CFTR was very sensitive in a manner independent of voltage to a pyrimido-pyrrolo-quinoxalinedione, $\mathrm{PPQ}-102$, with an $\mathrm{IC}_{50}$ of approximately $90 \mathrm{nM}$ (Tradtrantip et al., 2009) and to a benzopyrimide-pyrrolo-oxazinedione, BPO-27, with $\mathrm{IC}_{50}$ values of approximately $8 \mathrm{nM}$ (Snyder et al., 2011) and 0.5 and $360 \mathrm{nM}$ from the intracellular and extracellular sides, respectively, by competition with ATP (Kim et al., 2015). A scorpion Leiurus quinquestriatus hebraeus venom peptide toxin, GaTx1, also voltage-independently blocks CFTR very potently from the intracellular side with an $\mathrm{IC}_{50}$ of 48 and $220 \mathrm{nM}$ in the presence of intracellular ATP at 0.2 and $1 \mathrm{mM}$, respectively, without affecting $\mathrm{ClC}-2$ and $\mathrm{CaCC}$ (Fuller et al., 2007).

CFTR currents are suppressed by other various substances, including the following: arachidonic acid, in a voltage-independent manner, with an $\mathrm{IC}_{50}$ of 3.56.5 $\mu \mathrm{M}$ from the intracellular side (Linsdell, 2000; Zhou and Linsdell, 2007); suramin, in a voltage-dependent manner, with an $\mathrm{IC}_{50}$ of $2.8 \mu \mathrm{M}$ at $+40 \mathrm{mV}$ and $1 \mu \mathrm{M}$ at $-70 \mathrm{mV}$ from the intracellular side (Bachmann et al., 1999); and phloretin, with $\mathrm{IC}_{50}$ values of $195-252 \mu \mathrm{M}$ at $-60 \mathrm{mV}$ and $355-475 \mu \mathrm{M}$ at $+60 \mathrm{mV}$ from the extracellular side (Fan et al., 2001). Components of the antidiarrheal Chinese herb Regel Maxime, (-)-epigallocatechin-3gallate and (-)-epicatechin-3-gallate, were also reported to inhibit CFTR activity (Chen et al., 2015).

CFTR current was insensitive to a number of known anion channel blockers, including $\mathrm{Cd}^{2+}$ (Wang et al., 2009; Infield et al., 2016), Gd ${ }^{3+}$ (Hazama et al., 2000), DCPIB (Decher et al., 2001), methadone (Cuppoletti et al., 2013, 2014), and mefloquine (Maertens et al., 2000). In contrast to GaTx1, GaTx2 failed to inhibit CFTR activity (Thompson et al., 2009).

CFTR activity has been shown to be enhanced, but not suppressed, by several compounds. A flavonoid, genistein, which is a known potent PTK inhibitor, can PTK-independently activate CFTR from the extracellular (not intracellular) side (French et al., 1997; Wang et al., 1998; Zhou et al., 1998; Niisato et al., 1999). CFTR was also activated by some herbs such as menthol (Morise et al., 2010), which is a cyclic terpene alcohol produced by the peppermint herb; mashiningan (Harada et al., 2017), which is a Japanese herbal (Kampo) medicine used to treat constipation; as well as Junchoto, another Kampo medicine empirically prescribed for chronic constipation (Numata et al., 2018).

\section{E. Calcium-Activated Chloride Channel Blockers}

$\mathrm{CaCC}$ activity was reported to be blocked by a large variety of chemical substances (Frings et al., 2000). Among them, NFA is the most effective blocker for CaCC (see Table 2). Micromolar $\mathrm{IC}_{50}$ values were observed in vascular smooth muscle cells $(6.6 \mu \mathrm{M}$ in
Hogg et al., 1994b; $2 \mu \mathrm{M}$ in Greenwood and Large, 1995), epithelial cells (7.6 $\mu \mathrm{M}$ in $\mathrm{Qu}$ et al., 2003a; approximately $2.5 \mu \mathrm{M}$ in Romanenko et al., 2010), and melatonin-secreting pinealocytes $(2.6 \mu \mathrm{M}$ in Yamamura et al., 2018) as well as in TMEM16A-overexpressing Chinese hamster ovary (CHO) cells $(7.4 \mu \mathrm{M}$ in Liu et al., 2015). Deca-micromolar (10-99 $\mu \mathrm{M}) \mathrm{IC}_{50}$ values were observed in Xenopus oocytes ( $17 \mu \mathrm{M}$ in White and Aylwin, 1990; 10.1 $\mu \mathrm{M}$ in Qu and Hartzell, 2001), dog and cow tracheal epithelial cells $(20 \mu \mathrm{M}$ in Chao and Mochizuki, 1992), frog olfactory neurons (44 $\mu \mathrm{M}$ in Kleene, 1993), guinea pig tracheal smooth muscle cells (10 $\mu \mathrm{M}$ in Henmi et al., 1996), and rat vascular smooth muscle cells (26 $\mu \mathrm{M}$ in Robertson, 1998) as well as in TMEM16A-overexpressing HEK293 cells $(12 \mu \mathrm{M}$ in Bradley et al., 2014), whereas a sub-millimolar $\mathrm{IC}_{50}$ value was found in coronary smooth muscle cells (159 $\mu \mathrm{M}$ in Ledoux et al., 2005). However, NFA can also block VSOR with an $\mathrm{IC}_{50}$ of $320 \mu \mathrm{M}$ in HeLa cells (Sato-Numata et al., 2016) and $55 \mu \mathrm{M}$ in HEK293 cells (Friard et al., 2017) as well as ASOR with an $\mathrm{IC}_{50}$ of $11 \mu \mathrm{M}$ in HeLa cells (Sato-Numata et al., 2016).

Although FFA, which was initially identified as an anti-inflammatory agent (Winder et al., 1963), is commonly used to block nonselective cation channels, it is also an effective CaCC blocker, exhibiting decamicromolar $\mathrm{IC}_{50}$ values $(20-60 \mu \mathrm{M})$ in native nonneuronal cells (White and Aylwin, 1990; Chao and Mochizuki, 1992; Greenwood and Large, 1995) and in TMEM16A-overexpressing CHO cells (Liu et al., 2015) but with an $\mathrm{IC}_{50}$ of $108 \mu \mathrm{M}$ in frog olfactory neurons (Kleene, 1993).

CaCC activity is also sensitive to a number of, although not all, conventional anion channel blockers. DIDS voltage-dependently suppresses CaCC currents with deca-micromolar $\mathrm{IC}_{50}$ values $(16.5-48 \mu \mathrm{M})$ in some native cells (Baron et al., 1991; Qu and Hartzell, 2001; Romanenko et al., 2010) or with sub-millimolar IC $_{50}$ values (100-750 $\mu \mathrm{M})$ in other native cells (Akasu et al., 1990; Wladkowski et al., 1998; Hogg et al., 1994a) as well as in Tmem16a-transfected CHO cells (Liu et al., 2015). Another stilbene-derivative (SITS) is a less potent blocker for CaCC, exhibiting an $\mathrm{IC}_{50}$ of 300 $2000 \mu \mathrm{M}$ (Akasu et al., 1990; Hussy, 1991; Wang et al., 1992; Hogg et al., 1994a). NPPB inhibits CaCC currents with an $\mathrm{IC}_{50}$ of $22-68 \mu \mathrm{M}$ in Xenopus oocytes (Wu and Hamill, 1992) and $64.1 \mu \mathrm{M}$ in CHO cells stably expressing TMEM16A (Liu et al., 2015). DPC was found to block CaCC with sub-millimolar IC $_{50}$ values $(111-320 \mu \mathrm{M})$ in several cell types (Baron et al., 1991; Chao and Mochizuki, 1992; Qu and Hartzell, 2001). 9-AC also inhibits $\mathrm{CaCC}$ with deca-micromolar to sub-millimolar $\mathrm{IC}_{50}$ values of $18.3 \mu \mathrm{M}$ (Qu and Hartzell, 2001), approximately $30 \mu \mathrm{M}$ (Romanenko et al., 2010), $117 \mu \mathrm{M}$ (Baron et al., 1991), and $650 \mu \mathrm{M}$ (Hogg et al., 1994a) and with an $\mathrm{IC}_{50}$ of $58 \mu \mathrm{M}$ in HEK293 cells overexpressing TMEM16A (Bradley et al., 2014). 
More potent CaCC blockers were recently identified by high-throughput screening (De La Fuente et al., 2008; Namkung et al., 2011). T16 $\mathrm{A}_{\text {inh }^{-} \mathrm{A} 01}$ voltageindependently inhibits $\mathrm{CaCC}$ with a low micromolar $\mathrm{IC}_{50}$ value $(3.4 \mu \mathrm{M})$ in rabbit urethral interstitial cells of Cajal (Fedigan et al., 2017) and with sub-micromolar to micromolar $\mathrm{IC}_{50}$ values in TMEM16A-overexpressing cells $(1.1 \mu \mathrm{M}$ in Namkung et al., 2011; $1.5 \mu \mathrm{M}$ in Bradley et al., 2014; $0.31 \mu \mathrm{M}$ in Liu et al., 2015). $\mathrm{CaCC}_{\text {inh }}-\mathrm{A} 01$ also voltage-independently blocks $\mathrm{CaCC}$ with micromolar $\mathrm{IC}_{50}$ values in both native cells $(1.2 \mu \mathrm{M}$ in Fedigan et al., 2017) and TMEM16A-overexpressing cells (2.1 $\mu \mathrm{M}$ in Namkung et al., 2011; $1.7 \mu \mathrm{M}$ in Bradley et al., 2014; $7.8 \mu \mathrm{M}$ in Liu et al., 2015). Although both $\mathrm{CaCC}$ antagonists were reported to fail to suppress VSOR activity in pancreatic ductal adenocarcinoma (Sauter et al., 2015), the specificity of these inhibitors has not been well studied, especially in other cell types, and thus remains questionable (see Table 2). In fact, $\mathrm{T} 16 \mathrm{~A}_{\text {inh }}$-A01 was recently found to block not only L-type voltage-gated $\mathrm{Ca}^{2+}$ channels with an $\mathrm{IC}_{50}$ of $0.05 \mu \mathrm{M}$ in vascular smooth muscle $\mathrm{A} 7 \mathrm{r} 5$ cells (Boedtkjer et al., 2015) but also VSOR with an $\mathrm{IC}_{50}$ of $5.5 \mu \mathrm{M}$ in HEK293 cells (Friard et al., 2017). In addition, CaCC inh-A01 $(10 \mu \mathrm{M})$ increased the intracellular $\mathrm{Ca}^{2+}$ level in HEK293 cells (Kunzelmann et al., 2012).

Other potent $\mathrm{CaCC}$ blockers include tannic acid, $\mathrm{N}$ ((4-methyoxy)-2-naphthyl)-5-nitroanthranilic acid, and 2-(4-chloro-2-methylphenoxy)- $N$-[(2-methoxyphenyl)methylideneamino]-acetamide. The $\mathrm{IC}_{50}$ values of tannic acid for CaCC inhibition were reported to be $3.1 \mu \mathrm{M}$ in T84 cells and $11.2 \mu \mathrm{M}$ in human bronchial epithelial cells (Namkung et al., 2010) and $25.68 \mu \mathrm{M}$ in stably Tmem16a-transfected CHO cells (Liu et al., 2015). $N$-((4-methyoxy)-2-naphthyl)-5-nitroanthranilic acid was found to more potently block $\mathrm{CaCC}$ currents in Xenopus oocytes, in which xTMEM16A is endogenously expressed with an $\mathrm{IC}_{50}$ of $0.08 \mu \mathrm{M}$ and in transiently hTMEM16A-expressing HEK293 cells with an $\mathrm{IC}_{50}$ of $1.27 \mu \mathrm{M}$ without affecting CFTR and ClC-2 currents at 10-30 $\mu \mathrm{M}$ (Oh et al., 2013). 2-(4-Chloro-2-methylphenoxy)- $N$-[(2-methoxyphenyl)methylideneamino]-acetamide was observed to completely and voltage-independently inhibit $\mathrm{CaCC}$ currents in normal human nasal epithelial cells and hTMEM16A-overexpressing FRT cells with submicromolar potency $\left(\mathrm{IC}_{50}=0.11 \mu \mathrm{M}\right)$ without inhibiting mClC-2 and hCFTR currents at $10 \mu \mathrm{M}$ (Seo et al., 2016).

A putative VSOR blocker, NS3728, was found to equipotently block $\mathrm{CaCC}$ with $\mathrm{IC}_{50}$ values of $2.1 \mu \mathrm{M}$ at $-95 \mathrm{mV}$ and $0.7 \mu \mathrm{M}$ at $+50 \mathrm{mV}$ in ELA cells (Klausen et al., 2007) and $1.3 \mu \mathrm{M}$ in TMEM16A-overexpressing HEK293 cells (Sauter et al., 2015). CaCC activity was also sensitive to known CFTR blockers, glibenclamide and GlyH-101. IC $_{50}$ values of glibenclamide for CaCC blocking were $61.5 \mu \mathrm{M}$ at $+50 \mathrm{mV}$ and $69.9 \mu \mathrm{M}$ at $-100 \mathrm{mV}$ in cardiac myocytes (Yamazaki and Hume, 1997), and the $\mathrm{IC}_{50}$ of GlyH-101 was $3.4 \mu \mathrm{M}$ in kidney tubular cells (Melis et al., 2014). In contrast, another CFTR blocker, CFTR inh -172, was observed to be ineffective in blocking native CaCC currents at $\geq 5 \mu \mathrm{M}$ in kidney tubular cells (Melis et al., 2014) and TMEM16Aassociated CaCC currents in stable-transfected FRT cells at $5-100 \mu \mathrm{M}$ (Caputo et al., 2008). CaCC activity was reported to be insensitive to VSOR blocker DCPIB (Decher et al., 2001), Maxi-Cl blocker Gd ${ }^{3+}$ (Waniishi et al., 1998), and phloretin (Fan et al., 2001), which is an effective blocker for VSOR (Fan et al., 2001) and ASOR (Wang et al., 2007; Sato-Numata et al., 2014). Although tamoxifen was found to be inhibitory for CaCC solely in the calf bovine pulmonary artery endothelial cell line (Nilius et al., 1997b), subsequent studies failed to show any inhibitory effect on CaCC activity in human pancreatic ductal adenocarcinoma HPAF cells (Winpenny et al., 1998) and in mouse kidney inner medullary collecting duct cells (Qu et al., 2003a).

\section{F. Acid-Sensitive Outwardly Rectifying Anion Channel Blockers}

The most effective blocker of ASOR currently available is DIDS applied from the extracellular site. The $\mathrm{IC}_{50}$ values of DIDS for ASOR were reported to be submicromolar $(0.12 \mu \mathrm{M}$ in Wang et al., 2007; $0.5 \mu \mathrm{M}$ in Capurro et al., 2015) or micromolar $(2.9 \mu \mathrm{M}$ in Lambert and Oberwinkler, 2005; $4.7 \mu \mathrm{M}$ in Sato-Numata et al., 2014) (see Table 2). However, it is noted that DIDS can also block VSOR, Maxi-Cl, and $\mathrm{CaCC}$, although at higher concentrations, as described above. Blocking effects of DIDS on ASOR and Maxi-Cl are voltage independent, whereas those on VSOR and CaCC are voltage dependent. ASOR activity was highly sensitive to suramin and DIOA with $\mathrm{IC}_{50}$ values of $0.05 \mu \mathrm{M}$ and $1.9 \mu \mathrm{M}$, respectively (Sato-Numata et al., 2016). Notably, suramin blocked ASOR at concentrations two orders of magnitude lower than VSOR and CFTR, and this blocking action was exerted in a voltage-independent manner, in contrast to its voltage-dependent blocking action on VSOR (Sato-Numata et al., 2016) and CFTR (Bachmann et al., 1999). In addition, ASOR activity was suppressed by NFA and arachidonic acid with $\mathrm{IC}_{50}$ values of $11.0 \mu \mathrm{M}$ and $8.9 \mu \mathrm{M}$, respectively (Sato-Numata et al., 2016). It is worth mentioning that ASOR is one to two orders of magnitude more sensitive to NFA and arachidonic acid as well. ASOR is also inhibited by phloretin with an $\mathrm{IC}_{50}$ of 17.5-22.0 $\mu \mathrm{M}$ (Wang et al., 2007; SatoNumata et al., 2014) and glibenclamide with an $\mathrm{IC}_{50}$ of approximately $100 \mu \mathrm{M}$ (Yamamoto and Ehara, 2006).

ASOR activity was not blocked by a ClC-2 blocker, $\mathrm{Cd}^{2+}(1 \mathrm{mM})$, as shown in Fig. 12B. ASOR currents were found to be insensitive to a number of other anion channel blockers, including DCPIB, NPPB, CBX, Gd ${ }^{3+}$, and mefloquine (Sato-Numata et al., 2016) as well as tamoxifen (Nobles et al., 2004; Yamamoto and Ehara, 2006; Sato-Numata et al., 2016). Extracellular application of ATP and a P2 receptor blocker, 
pyridoxalphosphate-6-azophenyl-2', $4^{\prime}$-disulfonic acid (which are known to block VSOR), exerted no blocking action on ASOR (Sato-Numata et al., 2016).

\section{G. Their Pharmacological Distinctions}

Distinguishing VAACs and VCACs from each other must eventually, in principle, be done by investigating differences in their phenotypic biophysical properties and by observing the effects of abolition or modification of genes for their molecular identities. However, when specific and selective blockers are available, such pharmacological agents would provide the quickest and easiest tools to detect the involvement of particular channels in biologic events.

The selectivity and specificity of chemical substances for VAACs and VCACs are summarized by providing the $\mathrm{IC}_{50}$ values together with voltage dependence for their blocking action in Table 2. Based on the information listed in Table 2, the pharmacological distinctions between these anion channels can be summarized as follows. $\mathrm{Cd}^{2+}$ is the most selective blocker for ClC-2. However, effects of $\mathrm{Cd}^{2+}$ on $\mathrm{CaCC}$ activity have not been elucidated, maybe at least in part because $\mathrm{Cd}^{2+}$ often blocks channel-mediated $\mathrm{Ca}^{2+}$ entry, thereby affecting the intracellular $\mathrm{Ca}^{2+}$ level. To distinguish ClC-2 from $\mathrm{CaCC}$, its GaTx2 sensitivity would provide additional evidence. Simply, DCPIB sensitivity can discriminate VSOR from the others. Micromolar sensitivity to $\mathrm{Gd}^{3+}$ may distinguish Maxi-Cl from the others. When the anion channel in question exhibits deca-micromolar to sub-millimolar sensitivity to $\mathrm{Gd}^{3+}$, its insensitivity to DCPIB or phloretin provides a good indication for Maxi-Cl to discriminate from VSOR. CFTR inh $^{-172}$ is not specific to CFTR. Among the chemicals listed in Table 2, GaTx1 is likely the most potent blocker for CFTR. However, GaTx1 sensitivity of VSOR, Maxi-Cl, and ASOR has not yet been reported. To distinguish CFTR from the others, its sub-micromolar sensitivity to NPPB or micromolar sensitivity to glibenclamide is indispensably to be examined in combination with GaTx1 sensitivity. Although $\mathrm{CaCC}_{\text {inh }}-\mathrm{A} 01$ and $\mathrm{T} 16 \mathrm{~A}_{\text {inh }^{-}}$ $\mathrm{A} 01$ are purportedly expected to be CaCC-selective blockers, information about the sensitivity of ClC-2, Maxi-Cl, CFTR, and ASOR to both drugs is currently lacking. Furthermore, the sensitivity of VSOR to $\mathrm{T} 16 \mathrm{~A}_{\mathrm{inh}}-\mathrm{A} 01$ was reported in some, but not all, cell types. To distinguish CaCC from ClC-2, Maxi-Cl, CFTR, and ASOR, its micromolar sensitivity (if any) to NFA may thus provide additional evidence in combination with sensitivity to $\mathrm{CaCC}_{\mathrm{inh}}-\mathrm{A} 01$ and $\mathrm{T} 16 \mathrm{~A}_{\mathrm{inh}}-\mathrm{A} 01$. Submicromolar sensitivity to DIDS alone would provide supporting evidence for ASOR. Even when DIDS sensitivity ranges from micromolar to sub-millimolar concentrations, NPPB insensitivity may provide additional evidence for ASOR.

\section{Conclusions and Future Directions}

In relation to cell volume, there are two groups of anion channels: VAACs and VCACs. VAACs are directly activated by cell swelling and consist of inwardrectifier ClC-2, mild outward-rectifier VSOR (also called VRAC), and nonrectifying large-conductance Maxi-Cl. The molecular identities of core components of VSOR and Maxi-Cl were recently discovered by unbiased genome-wide approaches. However, some other as-yet-unknown regulatory components remain to be identified for whole VSOR and Maxi-Cl complexes. VCACs consist of cAMP-activated ohmic smallconductance CFTR activated by cAMP/PKA, strong outward-rectifier $\mathrm{CaCC}$ activated by cytosolic $\mathrm{Ca}^{2+}$, and another strong outward-rectifier activated by acid. Although CFTR and CaCC molecules were successfully identified, the molecular basis of ASOR has not yet been elucidated. A large number of chemical compounds have been known to block VAACs and VCACs with respective sub-micromolar to sub-millimolar IC $_{50}$ values. DCPIB is the sole selective blocker specific to VSOR. However, others exhibit, more or less, broad specificity or their specificity remains uncertain. Nevertheless, pharmacological distinctions of VAACs and VCACs from each other can be suitably attained by the combinatory application of two different blockers relatively specific to given anion channels. Discovery of more specific blockers requires future studies performed particularly on the basis of molecular understanding. Since these anion channels are prerequisitely involved not only in important physiologic functions but also in a variety of pathologic situations and human diseases such as ischemia/reperfusion injury and cancer, future findings of their highly specific blockers or activators would be beneficial for therapeutic purposes.

\section{Acknowledgments}

We thank Shigetoshi Oiki for pertinent discussion and Tsukasa Kawahara for the kind gift of some plasmids for NOXs.

\section{Authorship Contributions}

Participated in research design: Y. Okada, Sabirov.

Conducted experiments: T. Okada, Sato-Numata, Islam, AndoAkatsuka, Numata, Kubo, Shimizu, Kurbannazarova.

Performed data analysis: Y. Okada, T. Okada, Sato-Numata, Islam, Ando-Akatsuka, Numata, Shimizu, Sabirov.

Wrote or contributed to the writing of the manuscript: Y. Okada, Marunaka, Sabirov.

\section{References}

Abascal F and Zardoya R (2012) LRRC8 proteins share a common ancestor with pannexins, and may form hexameric channels involved in cell-cell communication. BioEssays 34:551-560.

Accardi A and Picollo A (2010) CLC channels and transporters: proteins with borderline personalities. Biochim Biophys Acta 1798:1457-1464.

Adomaviciene A, Smith KJ, Garnett H, and Tammaro P (2013) Putative pore-loops of TMEM16/anoctamin channels affect channel density in cell membranes. $J$ Physiol 591:3487-3505.

Ahluwalia J (2008a) Characterisation of electron currents generated by the human neutrophil NADPH oxidase. Biochem Biophys Res Commun 368:656-661. 
Ahluwalia J (2008b) Tamoxifen does not inhibit the swell activated chloride channel in human neutrophils during the respiratory burst. Biochem Biophys Res Commun 375:596-601.

Ai T, Bompadre SG, Sohma Y, Wang X, Li M, and Hwang TC (2004) Direct effects of 9 -anthracene compounds on cystic fibrosis transmembrane conductance regulator gating. Pflugers Arch 449:88-95.

Akanda N and Elinder F (2006) Biophysical properties of the apoptosis-inducing plasma membrane voltage-dependent anion channel. Biophys $J$ 90:4405-4417.

Akasu T, Nishimura T, and Tokimasa T (1990) Calcium-dependent chloride current in neurones of the rabbit pelvic parasympathetic ganglia. $J$ Physiol 422:303-320.

Akita T, Fedorovich SV, and Okada Y (2011) $\mathrm{Ca}^{2+}$ nanodomain-mediated component of swelling-induced volume-sensitive outwardly rectifying anion current triggered by autocrine action of ATP in mouse astrocytes. Cell Physiol Biochem 28: $1181-1190$

Akita T and Okada Y (2011) Regulation of bradykinin-induced activation of volumesensitive outwardly rectifying anion channels by $\mathrm{Ca}^{2+}$ nanodomains in mouse astrocytes. J Physiol 589:3909-3927.

Akita T and Okada Y (2014) Characteristics and roles of the volume-sensitive outwardly rectifying (VSOR) anion channel in the central nervous system. Neuroscience 275:211-231.

Allen MC, Newland C, Valverde MA, and Hardy SP (1998) Inhibition of ligand-gated cation-selective channels by tamoxifen. Eur J Pharmacol 354:261-269.

Almaça J, Tian Y, Aldehni F, Ousingsawat J, Kongsuphol P, Rock JR, Harfe BD, Schreiber R, and Kunzelmann K (2009) TMEM16 proteins produce volumeregulated chloride currents that are reduced in mice lacking TMEM16A. $J$ Biol Chem 284:28571-28578.

Anderson MP, Gregory RJ, Thompson S, Souza DW, Paul S, Mulligan RC, Smith AE, and Welsh MJ (1991) Demonstration that CFTR is a chloride channel by alteration of its anion selectivity. Science 253:202-205.

Ando-Akatsuka Y, Abdullaev IF, Lee EL, Okada Y, and Sabirov RZ (2002) Downregulation of volume-sensitive $\mathrm{Cl}^{-}$channels by CFTR is mediated by the second nucleotide-binding domain. Pflugers Arch 445:177-186.

Ando-Akatsuka Y, Shimizu T, Numata T, and Okada Y (2012) Involvements of the $\mathrm{ABC}$ protein $\mathrm{ABCF} 2$ and $\alpha$-actinin- 4 in regulation of cell volume and anion channels in human epithelial cells. J Cell Physiol 227:3498-3510.

Arreola J, Hallows KR, and Knauf PA (1995) Volume-activated chloride channels in HL-60 cells: potent inhibition by an oxonol dye. Am $J$ Physiol 269 C1063-C1072.

Auzanneau C, Thoreau V, Kitzis A, and Becq F (2003) A novel voltage-dependent chloride current activated by extracellular acidic $\mathrm{pH}$ in cultured rat Sertoli cells. $J$ Biol Chem 278:19230-19236.

Babinski K, Catarsi S, Biagini G, and Séguéla P (2000) Mammalian ASIC2a and ASIC3 subunits co-assemble into heteromeric proton-gated channels sensitive to $\mathrm{Gd}^{3+}$ J Biol Chem 275:28519-28525.

Bachmann A, Russ U, and Quast U (1999) Potent inhibition of the CFTR chloride channel by suramin. Naunyn Schmiedebergs Arch Pharmacol 360:473-476.

Bader CR, Bertrand D, and Schwartz EA (1982) Voltage-activated and calciumactivated currents studied in solitary rod inner segments from the salamander retina. J Physiol 331:253-284.

Bajnath RB, Groot JA, de Jonge HR, Kansen M, and Bijman J (1993) Calcium ionophore plus excision induce a large conductance chloride channel in membran patches of human colon carcinoma cells HT-29cl.19A. Experientia 49:313-316.

Bakhramov A, Fenech C, and Bolton TB (1995) Chloride current activated by hypotonicity in cultured human astrocytoma cells. Exp Physiol 80:373-389.

Barman PP, Choisy SC, Gadeberg HC, Hancox JC, and James AF (2011) Cardiac ion channel current modulation by the CFTR inhibitor GlyH-101. Biochem Biophys Res Commun 408:12-17.

Baron A, Pacaud P, Loirand G, Mironneau C, and Mironneau J (1991) Pharmacological block of $\mathrm{Ca}(2+)$-activated $\mathrm{Cl}^{-}$current in rat vascular smooth muscle cells in short-term primary culture. Pflugers Arch 419:553-558.

Barrière H, Belfodil R, Rubera I, Tauc M, Poujeol C, Bidet M, and Poujeol P (2003) CFTR null mutation altered cAMP-sensitive and swelling-activated $\mathrm{Cl}^{-}$currents in primary cultures of mouse nephron. Am J Physiol Renal Physiol 284:F796-F811.

Barro-Soria R, Aldehni F, Almaça J, Witzgall R, Schreiber R, and Kunzelmann K (2010) ER-localized bestrophin 1 activates $\mathrm{Ca}^{2+}$-dependent ion channels TMEM16A and SK4 possibly by acting as a counterion channel. Pflugers Arch $\mathbf{4 5 9}$ 485-497.

Barros LF, Hermosilla T, and Castro J (2001) Necrotic volume increase and the early physiology of necrosis. Comp Biochem Physiol A Mol Integr Physiol 130:401-409.

Becq F, Fanjul M, Mahieu I, Berger Z, Gola M, and Hollande E (1992) Anion channels in a human pancreatic cancer cell line (Capan-1) of ductal origin. Pflugers Arch 420:46-53.

Bedard K and Krause KH (2007) The NOX family of ROS-generating NADPH oxidases: physiology and pathophysiology. Physiol Rev 87:245-313.

Behe P, Foote JR, Levine AP, Platt CD, Chou J, Benavides F, Geha RS, and Segal AW (2017) The LRRC8A mediated "swell activated" chloride conductance is dispensable for vacuolar homeostasis in neutrophils. Front Pharmacol 8:262.

Belfodil R, Barrière H, Rubera I, Tauc M, Poujeol C, Bidet M, and Poujeol P (2003) CFTR-dependent and -independent swelling-activated $\mathrm{K}^{+}$currents in primary cultures of mouse nephron. Am J Physiol Renal Physiol 284:F812-F828.

Bell PD, Lapointe JY, Sabirov R, Hayashi S, Peti-Peterdi J, Manabe K, Kovacs G, and Okada Y (2003) Macula densa cell signaling involves ATP release through a maxi anion channel. Proc Natl Acad Sci USA 100:4322-4327.

Benedetto R, Sirianant L, Pankonien I, Wanitchakool P, Ousingsawat J, Cabrita I, Schreiber R, Amaral M, and Kunzelmann K (2016) Relationship between TMEM16A/anoctamin 1 and LRRC8A. Pflugers Arch 468:1751-1763.

Benfenati V, Caprini M, Nicchia GP, Rossi A, Dovizio M, Cervetto C, Nobile M, and Ferroni S (2009) Carbenoxolone inhibits volume-regulated anion conductance in cultured rat cortical astroglia. Channels (Austin) 3:323-336.
Bengele HH, Graber ML, and Alexander EA (1983) Effect of respiratory acidosis on acidification by the medullary collecting duct. Am J Physiol 244:F89-F94.

Bengele HH, Schwartz JH, McNamara ER, and Alexander EA (1986) Effect of buffer infusion during acute respiratory acidosis. Am J Physiol 250:F115-F119.

Best L and Brown PD (2009) Studies of the mechanism of activation of the volumeregulated anion channel in rat pancreatic beta-cells. J Membr Biol 230:83-91.

Bettendorff L, Kolb HA, and Schoffeniels E (1993) Thiamine triphosphate activates an anion channel of large unit conductance in neuroblastoma cells. J Membr Biol 136:281-288

Biagi BA and Enyeart JJ (1990) Gadolinium blocks low- and high-threshold calcium currents in pituitary cells. Am J Physiol 259:C515-C520.

Billig GM, Pál B, Fidzinski P, and Jentsch TJ (2011) $\mathrm{Ca}^{2+}$-activated $\mathrm{Cl}^{-}$currents are dispensable for olfaction. Nat Neurosci 14:763-769.

Blaesse P, Airaksinen MS, Rivera C, and Kaila K (2009) Cation-chloride cotransporters and neuronal function. Neuron 61:820-838.

Blaisdell CJ, Edmonds RD, Wang XT, Guggino S, and Zeitlin PL (2000) pH-regulated chloride secretion in fetal lung epithelia. Am J Physiol Lung Cell Mol Physiol 278: L1248-L1255.

Blatz AL and Magleby KL (1983) Single voltage-dependent chloride-selective channels of large conductance in cultured rat muscle. Biophys $J$ 43:237-241.

Blatz AL and Magleby KL (1985) Single chloride-selective channels active at resting membrane potentials in cultured rat skeletal muscle. Biophys $J$ 47:119-123.

Boedtkjer DM, Kim S, Jensen AB, Matchkov VM, and Andersson KE (2015) New selective inhibitors of calcium-activated chloride channels - T16A(inh) -A01, CaCC (inh) -A01 and MONNA - what do they inhibit? $\mathrm{Br} J$ Pharmacol 172:4158-4172.

Boese SH, Kinne RK, and Wehner F (1996) Single-channel properties of swellingactivated anion conductance in rat inner medullary collecting duct cells. Am J Physiol 271:F1224-F1233.

Bolotina V, Borecký J, Vlachová V, Baudysová M, and Vyskocil F (1987) Voltagedependent chloride channels with several substates in excised patches from mouse neuroblastoma cells. Neurosci Lett 77:298-302.

Bond TD, Ambikapathy S, Mohammad S, and Valverde MA (1998) Osmosensitive C1 currents and their relevance to regulatory volume decrease in human intestinal T84 cells: outwardly vs. inwardly rectifying currents. $J$ Physiol 511:45-54.

Bösl MR, Stein V, Hübner C, Zdebik AA, Jordt SE, Mukhopadhyay AK, Davidoff MS, Holstein AF, and Jentsch TJ (2001) Male germ cells and photoreceptors, both dependent on close cell-cell interactions, degenerate upon $\mathrm{ClC}-2 \mathrm{Cl}(-)$ channel disruption. EMBO J 20:1289-1299.

Bosma MM (1989) Anion channels with multiple conductance levels in a mouse B lymphocyte cell line. J Physiol 410:67-90.

Bradley E, Fedigan S, Webb T, Hollywood MA, Thornbury KD, McHale NG, and Sergeant GP (2014) Pharmacological characterization of TMEM16A currents. Channels (Austin) 8:308-320.

Braunstein GM, Roman RM, Clancy JP, Kudlow BA, Taylor AL, Shylonsky VG, Jovov B, Peter K, Jilling T, Ismailov II, et al. (2001) Cystic fibrosis transmembrane conductance regulator facilitates ATP release by stimulating a separate ATP release channel for autocrine control of cell volume regulation. J Biol Chem $\mathbf{2 7 6}$ : $6621-6630$.

Browe DM and Baumgarten CM (2004) Angiotensin II (AT1) receptors and NADPH oxidase regulate $\mathrm{Cl}^{-}$current elicited by beta1 integrin stretch in rabbit ventricular myocytes. J Gen Physiol 124:273-287.

Browe DM and Baumgarten CM (2006) EGFR kinase regulates volume-sensitive chloride current elicited by integrin stretch via PI-3K and NADPH oxidase in ventricular myocytes. J Gen Physiol 127:237-251.

Brown PD, Greenwood SL, Robinson J, and Boyd RD (1993) Chloride channels of high conductance in the microvillous membrane of term human placenta. Placenta 14:103-115.

Bryan-Sisneros A, Sabanov V, Thoroed SM, and Doroshenko P (2000) Dual role of ATP in supporting volume-regulated chloride channels in mouse fibroblasts. Biochim Biophys Acta 1468:63-72.

Caci E, Caputo A, Hinzpeter A, Arous N, Fanen P, Sonawane N, Verkman AS, Ravazzolo R, Zegarra-Moran O, and Galietta LJ (2008) Evidence for direct CFTR inhibition by CFTR(inh)-172 based on Arg347 mutagenesis. Biochem J 413: 135-142

Cahalan MD and Lewis RS (1988) Role of potassium and chloride channels in volume regulation by T lymphocytes. Soc Gen Physiol Ser 43:281-301.

Cannon CL, Basavappa S, and Strange K (1998) Intracellular ionic strength regulates the volume sensitivity of a swelling-activated anion channel. Am J Physiol 275:C416-C422.

Capurro V, Gianotti A, Caci E, Ravazzolo R, Galietta LJ, and Zegarra-Moran O (2015) Functional analysis of acid-activated $\mathrm{Cl}^{-}$channels: properties and mechanisms of regulation. Biochim Biophys Acta 1848:105-114.

Caputo A, Caci E, Ferrera L, Pedemonte N, Barsanti C, Sondo E, Pfeffer U, Ravazzolo R, Zegarra-Moran O, and Galietta LJ (2008) TMEM16A, a membrane protein associated with calcium-dependent chloride channel activity. Science $\mathbf{3 2 2}$ $590-594$

Carton I, Hermans D, and Eggermont J (2003) Hypotonicity induces membrane protrusions and actin remodeling via activation of small GTPases Rac and Cdc42 in Rat-1 fibroblasts. Am J Physiol Cell Physiol 285:C935-C944.

Catalán M, Cornejo I, Figueroa CD, Niemeyer MI, Sepúlveda FV, and Cid LP (2002) ClC-2 in guinea pig colon: mRNA, immunolabeling, and functional evidence for surface epithelium localization. Am J Physiol Gastrointest Liver Physiol 283: G1004-G1013.

Cater RJ, Ryan RM, and Vandenberg RJ (2016) The split personality of glutamate transporters: a chloride channel and a transporter. Neurochem Res 41:593-599. Chan BS, Bao Y, and Schuster VL (2002) Role of conserved transmembrane cationic amino acids in the prostaglandin transporter PGT. Biochemistry 41:9215-9221.

Chao AC and Mochizuki H (1992) Niflumic and flufenamic acids are potent inhibitors of chloride secretion in mammalian airway. Life Sci 51:1453-1457. 
Chen L, Wang L, Zhu L, Nie S, Zhang J, Zhong P, Cai B, Luo H, and Jacob TJ (2002) Cell cycle-dependent expression of volume-activated chloride currents in nasopharyngeal carcinoma cells. Am J Physiol Cell Physiol 283:C1313-C1323.

Chen L, Yu B, Zhang Y, Gao X, Zhu L, Ma T, and Yang H (2015) Bioactivity-guided fractionation of an antidiarrheal Chinese herb Rhodiola kirilowii (Regel) Maxim reveals (-)-epicatechin-3-gallate and (-)-epigallocatechin-3-gallate as inhibitors of cystic fibrosis transmembrane conductance regulator. PLoS One 10:e119122.

Chen MF and Chen TY (2001) Different fast-gate regulation by external $\mathrm{Cl}\left({ }^{-}\right)^{-}$and $\mathrm{H}$ $(+)$ of the muscle-type ClC chloride channels. J Gen Physiol 118:23-32.

Chesler M and Kaila K (1992) Modulation of $\mathrm{pH}$ by neuronal activity. Trends Neurosci 15:396-402.

Chesnoy-Marchais D and Fritsch J (1994) Activation of hyperpolarization and atypical osmosensitivity of a $\mathrm{Cl}^{-}$current in rat osteoblastic cells. J Membr Biol $\mathbf{1 4 0}$ 173-188.

Chien LT and Hartzell HC (2007) Drosophila bestrophin-1 chloride current is dually regulated by calcium and cell volume. J Gen Physiol 130:513-524.

Chien LT, Zhang ZR, and Hartzell HC (2006) Single $\mathrm{Cl}^{-}$channels activated by $\mathrm{Ca}^{2+}$ in Drosophila S2 cells are mediated by bestrophins. J Gen Physiol 128:247-259.

Chipperfield AR and Harper AA (2000) Chloride in smooth muscle. Prog Biophys Mol Biol 74:175-221.

Choi H, Ettinger N, Rohrbough J, Dikalova A, Nguyen HN, and Lamb FS (2016) LRRC8A channels support TNF $\alpha$-induced superoxide production by Nox 1 which is required for receptor endocytosis. Free Radic Biol Med 101:413-423.

Clark S, Jordt SE, Jentsch TJ, and Mathie A (1998) Characterization of the hyperpolarization-activated chloride current in dissociated rat sympathetic neurons. J Physiol 506:665-678.

Coste B, Mathur J, Schmidt M, Earley TJ, Ranade S, Petrus MJ, Dubin AE, and Patapoutian A (2010) Piezo1 and Piezo2 are essential components of distinct mechanically activated cation channels. Science 330:55-60.

Coulombe A and Coraboeuf E (1992) Large-conductance chloride channels of newborn rat cardiac myocytes are activated by hypotonic media. Pflugers Arch 422: 143-150.

Coulombe A, Duclohier H, Coraboeuf E, and Touzet N (1987) Single chloridepermeable channels of large conductance in cultured cardiac cells of new-born rats. Eur Biophys J 14:155-162.

Crutzen R, Shlyonsky V, Louchami K, Virreira M, Hupkens E, Boom A, Sener A, Malaisse WJ, and Beauwens R (2012) Does NAD(P)H oxidase-derived $\mathrm{H}_{2} \mathrm{O}_{2}$ participate in hypotonicity-induced insulin release by activating VRAC in $\beta$-cells? Pflugers Arch 463:377-390.

Crutzen R, Virreira M, Markadieu N, Shlyonsky V, Sener A, Malaisse WJ, Beauwens $\mathrm{R}$, Boom A, and Golstein PE (2016) Anoctamin 1 (Ano1) is required for glucoseinduced membrane potential oscillations and insulin secretion by murine $\beta$-cells. Pflugers Arch 468:573-591.

Cunningham SA, Awayda MS, Bubien JK, Ismailov II, Arrate MP, Berdiev BK, Benos DJ, and Fuller CM (1995) Cloning of an epithelial chloride channel from bovine trachea. J Biol Chem 270:31016-31026.

Cuppoletti J, Chakrabarti J, Tewari K, and Malinowska DH (2013) Methadone but not morphine inhibits lubiprostone-stimulated $\mathrm{Cl}^{-}$currents in T84 intestinal cells and recombinant human ClC-2, but not CFTR $\mathrm{Cl}^{-}$currents. Cell Biochem Biophys 66:53-63.

Cuppoletti J, Chakrabarti J, Tewari KP, and Malinowska DH (2014) Differentiation between human ClC-2 and CFTR $\mathrm{Cl}^{-}$channels with pharmacological agents. $\mathrm{Am} J$ Physiol Cell Physiol 307:C479-C492.

Darby M, Kuzmiski JB, Panenka W, Feighan D, and MacVicar BA (2003) ATP released from astrocytes during swelling activates chloride channels. J Neurophysiol 89:1870-1877.

De La Fuente R, Namkung W, Mills A, and Verkman AS (2008) Small-molecule screen identifies inhibitors of a human intestinal calcium-activated chloride channel. Mol Pharmacol 73:758-768.

De Marchi U, Szabò I, Cereghetti GM, Hoxha P, Craigen WJ, and Zoratti M (2008) A maxi-chloride channel in the inner membrane of mammalian mitochondria. Biochim Biophys Acta 1777:1438-1448.

Decher N, Lang HJ, Nilius B, Brüggemann A, Busch AE, and Steinmeyer K (2001) DCPIB is a novel selective blocker of $\mathrm{I}(\mathrm{Cl}$,swell) and prevents swelling-induced shortening of guinea-pig atrial action potential duration. $\mathrm{Br} J$ Pharmacol 134 $1467-1479$

Deneka D, Sawicka M, Lam AKM, Paulino C, and Dutzler R (2018) Structure of a volume-regulated anion channel of the LRRC8 family. Nature 558:254-259.

Deng W, Baki L, and Baumgarten CM (2010a) Endothelin signalling regulates volume-sensitive $\mathrm{Cl}^{-}$current via NADPH oxidase and mitochondrial reactive oxygen species. Cardiovasc Res 88:93-100.

Deng W, Baki L, Yin J, Zhou H, and Baumgarten CM (2010b) HIV protease inhibitors elicit volume-sensitive $\mathrm{Cl}^{-}$current in cardiac myocytes via mitochondrial ROS. $J$ Mol Cell Cardiol 49:746-752.

Deng W, Mahajan R, Baumgarten CM, and Logothetis DE (2016) The ICl,swell inhibitor DCPIB blocks Kir channels that possess weak affinity for PIP2. Pflugers Arch 468:817-824.

Dezaki K, Tsumura T, Maeno E, and Okada Y (2000) Receptor-mediated facilitation of cell volume regulation by swelling-induced ATP release in human epithelial cells. Jpn J Physiol 50:235-241.

Díaz M, Bahamonde MI, Lock H, Muñoz FJ, Hardy SP, Posas F, and Valverde MA (2001) Okadaic acid-sensitive activation of $\mathrm{Maxi} \mathrm{Cl}(-)$ channels by triphenylethylene antioestrogens in C1300 mouse neuroblastoma cells. J Physiol 536:79-88.

Díaz M, Valverde MA, Higgins CF, Rucăreanu C, and Sepúlveda FV (1993) Volumeactivated chloride channels in HeLa cells are blocked by verapamil and dideoxyforskolin. Pflugers Arch 422:347-353.

Dick GM, Kong ID, and Sanders KM (1999) Effects of anion channel antagonists in canine colonic myocytes: comparative pharmacology of $\mathrm{Cl}^{-}, \mathrm{Ca}^{2+}$ and $\mathrm{K}^{+}$currents. Br J Pharmacol 127:1819-1831.
Diewald L, Rupp J, Dreger M, Hucho F, Gillen C, and Nawrath H (2002) Activation by acidic pH of CLC-7 expressed in oocytes from Xenopus laevis. Biochem Biophys Res Commun 291:421-424.

Do CW, Peterson-Yantorno K, Mitchell CH, and Civan MM (2004) cAMP-activated maxi-Cl(-) channels in native bovine pigmented ciliary epithelial cells. Am J Physiol Cell Physiol 287:C1003-C1011.

Docherty RJ (1988) Gadolinium selectively blocks a component of calcium current in rodent neuroblastoma x glioma hybrid (NG108-15) cells. J Physiol 398:33-47.

Doroshenko P (1998) Pervanadate inhibits volume-sensitive chloride current in bovine chromaffin cells. Pflugers Arch 435:303-309.

Doroshenko P and Neher E (1992) Volume-sensitive chloride conductance in bovine chromaffin cell membrane. J Physiol 449:197-218.

Doroshenko P, Penner R, and Neher E (1991) Novel chloride conductance in the membrane of bovine chromaffin cells activated by intracellular GTP $\gamma$ S. J Physiol 436:711-724.

Duan D, Ye L, Britton F, Horowitz B, and Hume JR (2000) A novel anionic inward rectifier in native cardiac myocytes. Circ Res 86:E63-E71.

Duran C, Qu Z, Osunkoya AO, Cui Y, and Hartzell HC (2012) ANOs 3-7 in the anoctamin/Tmem $16 \mathrm{Cl}^{-}$channel family are intracellular proteins. Am J Physiol Cell Physiol 302:C482-C493.

Dutta AK, Korchev YE, Shevchuk AI, Hayashi S, Okada Y, and Sabirov RZ (2008) Spatial distribution of maxi-anion channel on cardiomyocytes detected by smartpatch technique. Biophys $J$ 94:1646-1655.

Dutta AK, Okada Y, and Sabirov RZ (2002) Regulation of an ATP-conductive largeconductance anion channel and swelling-induced ATP release by arachidonic acid. $J$ Physiol 542:803-816.

Dutta AK, Sabirov RZ, Uramoto H, and Okada Y (2004) Role of ATP-conductive anion channel in ATP release from neonatal rat cardiomyocytes in ischaemic or hypoxic conditions. J Physiol 559:799-812.

Eggermont J (2004) Calcium-activated chloride channels: (un)known, (un)loved? Proc Am Thorac Soc 1:22-27.

Eggermont J, Trouet D, Carton I, and Nilius B (2001) Cellular function and control of volume-regulated anion channels. Cell Biochem Biophys 35:263-274.

Elinder F, Akanda N, Tofighi R, Shimizu S, Tsujimoto Y, Orrenius S, and Ceccatelli S (2005) Opening of plasma membrane voltage-dependent anion channels (VDAC) precedes caspase activation in neuronal apoptosis induced by toxic stimuli. Cell Death Differ 12:1134-1140.

Enz R, Ross BJ, and Cutting GR (1999) Expression of the voltage-gated chloride channel ClC-2 in rod bipolar cells of the rat retina. $J$ Neurosci 19:9841-9847.

Erickson GR, Northrup DL, and Guilak F (2003) Hypo-osmotic stress induces calcium-dependent actin reorganization in articular chondrocytes. Osteoarthritis Cartilage 11:187-197.

Estevez AY, Bond T, and Strange K (2001) Regulation of I( $\mathrm{Cl}_{1}$ ) in neuroblastoma cells by G protein signaling pathways. Am J Physiol Cell Physiol 281:C89-C98.

Fahlke C (2001) Ion permeation and selectivity in ClC-type chloride channels. Am $J$ Physiol Renal Physiol 280:F748-F757.

Fahlke C, Kortzak D, and Machtens JP (2016) Molecular physiology of EAAT anion channels. Pflugers Arch 468:491-502.

Fahlke C, Rhodes TH, Desai RR, and George AL Jr (1998) Pore stoichiometry of a voltage-gated chloride channel. Nature 394:687-690.

Falke LC and Misler S (1989) Activity of ion channels during volume regulation by clonal N1E115 neuroblastoma cells. Proc Natl Acad Sci USA 86:3919-3923.

Fan HT, Morishima S, Kida H, and Okada Y (2001) Phloretin differentially inhibits volume-sensitive and cyclic AMP-activated, but not Ca-activated, $\mathrm{Cl}(-)$ channels. $\mathrm{Br}$ J Pharmacol 133:1096-1106.

Fedigan S, Bradley E, Webb T, Large RJ, Hollywood MA, Thornbury KD, McHale NG, and Sergeant GP (2017) Effects of new-generation TMEM16A inhibitors on calcium-activated chloride currents in rabbit urethral interstitial cells of Cajal. Pflugers Arch 469:1443-1455.

Fine BP, Marques ES, and Hansen KA (1994) Calcium-activated sodium and chloride fluxes modulate platelet volume: role of $\mathrm{Ca}^{2+}$ stores. Am J Physiol 267: C1435-C1441.

Fischmeister R and Hartzell HC (2005) Volume sensitivity of the bestrophin family of chloride channels. J Physiol 562:477-491.

Földy C, Lee SH, Morgan RJ, and Soltesz I (2010) Regulation of fast-spiking basket cell synapses by the chloride channel ClC-2. Nat Neurosci 13:1047-1049.

Forshaw PJ, Lister T, and Ray DE (1993) Inhibition of a neuronal voltage-dependent chloride channel by the type II pyrethroid, deltamethrin. Neuropharmacology 32 105-111.

French PJ, Bijman J, Bot AG, Boomaars WE, Scholte BJ, and de Jonge HR (1997) Genistein activates CFTR $\mathrm{Cl}^{-}$channels via a tyrosine kinase- and protein phosphatase-independent mechanism. Am J Physiol 273:C747-C753.

Friard J, Tauc M, Cougnon M, Compan V, Duranton C, and Rubera I (2017) Comparative effects of chloride channel inhibitors on LRRC8/VRAC-mediated chloride conductance. Front Pharmacol 8:328.

Frings S, Reuter D, and Kleene SJ (2000) Neuronal $\mathrm{Ca}^{2+}$-activated $\mathrm{Cl}^{-}$channels-homing in on an elusive channel species. Prog Neurobiol 60:247-289.

Fritsch J and Edelman A (1997) Osmosensitivity of the hyperpolarization-activated chloride current in human intestinal T84 cells. Am J Physiol 272:C778-C786.

Fu ZJ, Li XZ, Wang QR, Shi L, Zhang LQ, and Pan XL (2013) Extracellular acidic $\mathrm{pH}$-activated, outward rectifying chloride currents can be regulated by reactive oxygen species in human THP-1 monocytes. Biochem Biophys Res Commun 432 : $701-706$

Fujii T, Takahashi Y, Takeshima H, Saitoh C, Shimizu T, Takeguchi N, and Sakai H (2015) Inhibition of gastric $\mathrm{H}^{+}, \mathrm{K}^{+}$-ATPase by 4-(2-butyl-6,7-dichloro-2-cyclopentylindan-1-on-5-yl)oxybutyric acid (DCPIB), an inhibitor of volume-regulated anion channel. Eur $J$ Pharmacol 765:34-41.

Fuller MD, Thompson CH, Zhang ZR, Freeman CS, Schay E, Szakács G, Bakos E, Sarkadi B, McMaster D, French RJ, et al. (2007) State-dependent inhibition of 
cystic fibrosis transmembrane conductance regulator chloride channels by a novel peptide toxin. J Biol Chem 282:37545-37555.

Fulmer SB, Schwiebert EM, Morales MM, Guggino WB, and Cutting GR (1995) Two cystic fibrosis transmembrane conductance regulator mutations have different effects on both pulmonary phenotype and regulation of outwardly rectified chloride currents. Proc Natl Acad Sci USA 92:6832-6836.

Furukawa T, Ogura T, Katayama Y, and Hiraoka M (1998) Characteristics of rabbit ClC-2 current expressed in Xenopus oocytes and its contribution to volume regulation. Am J Physiol 274:C500-C512.

Gaitán-Peñas H, Gradogna A, Laparra-Cuervo L, Solsona C, Fernández-Dueñas V, Barrallo-Gimeno A, Ciruela F, Lakadamyali M, Pusch M, and Estévez R (2016) Investigation of LRRC8-mediated volume-regulated anion currents in Xenopus oocytes. Biophys J 111:1429-1443.

Galietta LJ, Falzoni S, Di Virgilio F, Romeo G, and Zegarra-Moran O (1997) Characterization of volume-sensitive taurine- and $\mathrm{Cl}(-)$-permeable channels. Am $J$ Physiol 273:C57-C66.

Ghosh A, Khandelwal N, Kumar A, and Bera AK (2017) Leucine-rich repeatcontaining $8 \mathrm{~B}$ protein is associated with the endoplasmic reticulum $\mathrm{Ca}^{2+}$ leak in HEK293 cells [published correction appears in J Cell Sci (2018) 131]. J Cell Sci 130:3818-3828.

Gómez NM, Tamm ER, and Strau $\beta$ O (2013) Role of bestrophin-1 in store-operated calcium entry in retinal pigment epithelium. Pflugers Arch 465:481-495.

Gradogna A, Gavazzo P, Boccaccio A, and Pusch M (2017) Subunit-dependent oxidative stress sensitivity of LRRC8 volume-regulated anion channels. J Physiol 595: $6719-6733$.

Greenwood IA and Large WA (1995) Comparison of the effects of fenamates on Ca-activated chloride and potassium currents in rabbit portal vein smooth muscle cells. Br J Pharmacol 116:2939-2948.

Grimm C, Kraft R, Sauerbruch S, Schultz G, and Harteneck C (2003) Molecular and functional characterization of the melastatin-related cation channel TRPM3. J Biol Chem 278:21493-21501.

Groschner K and Kukovetz WR (1992) Voltage-sensitive chloride channels of large conductance in the membrane of pig aortic endothelial cells. Pflugers Arch 421: 209-217.

Groulx N, Boudreault F, Orlov SN, and Grygorczyk R (2006) Membrane reserves and hypotonic cell swelling. J Membr Biol 214:43-56.

Grubb S, Poulsen KA, Juul CA, Kyed T, Klausen TK, Larsen EH, and Hoffmann EK (2013) TMEM16F (anoctamin 6), an anion channel of delayed $\mathrm{Ca}(2+)$ activation. $J$ Gen Physiol 141:585-600.

Gründer S, Thiemann A, Pusch M, and Jentsch TJ (1992) Regions involved in the opening of CIC-2 chloride channel by voltage and cell volume. Nature 360:759-762.

Guggino WB (1993) Outwardly rectifying chloride channels and CF: a divorce and remarriage. J Bioenerg Biomembr 25:27-35.

Gustin MC, Martinac B, Saimi Y, Culbertson MR, and Kung C (1986) Ion channels in yeast. Science 233:1195-1197.

Hagiwara N, Masuda H, Shoda M, and Irisawa H (1992) Stretch-activated anion currents of rabbit cardiac myocytes. J Physiol 456:285-302.

Hagiwara S and Byerly L (1981) Calcium channel. Annu Rev Neurosci 4:69-125.

Hals GD, Stein PG, and Palade PT (1989) Single channel characteristics of a high conductance anion channel in "sarcoballs". J Gen Physiol 93: $385-410$.

Hanano T, Hara Y, Shi J, Morita H, Umebayashi C, Mori E, Sumimoto H, Ito Y, Mori $\mathrm{Y}$, and Inoue R (2004) Involvement of TRPM7 in cell growth as a spontaneously activated $\mathrm{Ca}^{2+}$ entry pathway in human retinoblastoma cells. J Pharmacol Sci $\mathbf{9 5}$ 403-419.

Harada Y, Iizuka S, Saegusa Y, Mogami S, Fujitsuka N, and Hattori T (2017) Mashiningan improves opioid-induced constipation in rats by activating cystic fibrosis transmembrane conductance regulator chloride channel. J Pharmacol Exp Ther 362:78-84.

Hardy SP, deFelipe C, and Valverde MA (1998) Inhibition of voltage-gated cationic channels in rat embryonic hypothalamic neurones and C1300 neuroblastoma cells by triphenylethylene antioestrogens. FEBS Lett 434:236-240.

Hardy SP and Valverde MA (1994) Novel plasma membrane action of estrogen and antiestrogens revealed by their regulation of a large conductance chloride channel. FASEB J 8:760-765.

Harrigan TJ, Abdullaev IF, Jourd'heuil D, and Mongin AA (2008) Activation of microglia with zymosan promotes excitatory amino acid release via volumeregulated anion channels: the role of NADPH oxidases. $J$ Neurochem 106 2449-2462.

Hart P, Warth JD, Levesque PC, Collier ML, Geary Y, Horowitz B, and Hume JR (1996) Cystic fibrosis gene encodes a cAMP-dependent chloride channel in heart. Proc Natl Acad Sci USA 93:6343-6348.

Hartzell C, Putzier I, and Arreola J (2005) Calcium-activated chloride channels. Annu Rev Physiol 67:719-758.

Hazama A, Fan HT, Abdullaev I, Maeno E, Tanaka S, Ando-Akatsuka Y, and Okada $\mathrm{Y}$ (2000) Swelling-activated, cystic fibrosis transmembrane conductance regulatoraugmented ATP release and $\mathrm{Cl}^{-}$conductances in murine C127 cells. J Physiol 523: $1-11$

Hazama A and Okada Y (1988) $\mathrm{Ca}^{2+}$ sensitivity of volume-regulatory $\mathrm{K}^{+}$and $\mathrm{Cl}^{-}$ channels in cultured human epithelial cells. J Physiol 402:687-702.

Hazama A, Shimizu T, Ando-Akatsuka Y, Hayashi S, Tanaka S, Maeno E, and Okada Y (1999) Swelling-induced, CFTR-independent ATP release from a human epithelial cell line: lack of correlation with volume-sensitive $\mathrm{Cl}(-)$ channels. $J$ Gen Physiol 114:525-533.

He J, Kargacin ME, Kargacin GJ, and Ward CA (2003) Tamoxifen inhibits $\mathrm{Na}^{+}$and $\mathrm{K}^{+}$currents in rat ventricular myocytes. Am J Physiol Heart Circ Physiol 285 H661-H668.

Hegedus T, Aleksandrov A, Mengos A, Cui L, Jensen TJ, and Riordan JR (2009) Role of individual $\mathrm{R}$ domain phosphorylation sites in CFTR regulation by protein kinase A. Biochim Biophys Acta 1788:1341-1349.
Hélix N, Strøbaek D, Dahl BH, and Christophersen P (2003) Inhibition of the endogenous volume-regulated anion channel (VRAC) in HEK293 cells by acidic di-aryl-ureas. J Membr Biol 196:83-94.

Henmi S, Imaizumi Y, Muraki K, and Watanabe M (1996) Time course of $\mathrm{Ca}(2+)$ dependent $\mathrm{K}^{+}$and $\mathrm{Cl}^{-}$currents in single smooth muscle cells of guinea-pig trachea. Eur J Pharmacol 306:227-236.

Hermosura MC, Monteilh-Zoller MK, Scharenberg AM, Penner R, and Fleig A (2002) Dissociation of the store-operated calcium current I (CRAC) and the Mg-nucleotideregulated metal ion current MagNuM. J Physiol 539:445-458.

Hoffmann EK, Lambert IH, and Pedersen SF (2009) Physiology of cell volume regulation in vertebrates. Physiol Rev 89:193-277.

Hoffmann EK and Pedersen SF (2006) Sensors and signal transduction pathways in vertebrate cell volume regulation. Contrib Nephrol 152:54-104

Hogg RC, Wang Q, and Large WA (1994a) Effects of Cl channel blockers on Ca-activated chloride and potassium currents in smooth muscle cells from rabbit portal vein. $\mathrm{Br} J$ Pharmacol 111:1333-1341.

Hogg RC, Wang Q, and Large WA (1994b) Action of niflumic acid on evoked and spontaneous calcium-activated chloride and potassium currents in smooth muscle cells from rabbit portal vein. $\mathrm{Br} J$ Pharmacol 112:977-984.

Holm JB, Grygorczyk R, and Lambert IH (2013) Volume-sensitive release of organic osmolytes in the human lung epithelial cell line A549: role of the 5-lipoxygenase. Am J Physiol Cell Physiol 305:C48-C60.

Huang F, Wong X, and Jan LY (2012a) International Union of Basic and Clinical Pharmacology. LXXXV: calcium-activated chloride channels. Pharmacol Rev 64: $1-15$.

Huang WC, Xiao S, Huang F, Harfe BD, Jan YN, and Jan LY (2012b) Calciumactivated chloride channels $(\mathrm{CaCCs})$ regulate action potential and synaptic re sponse in hippocampal neurons. Neuron 74:179-192.

Huber S, Schröppel B, Kretzler M, Schlöndorff D, and Horster M (1998) Single cell RT-PCR analysis of ClC-2 mRNA expression in ureteric bud tip. Am J Physiol 274: F951-F957.

Huber SM, Duranton C, Henke G, Van De Sand C, Heussler V, Shumilina E, Sandu CD, Tanneur V, Brand V, Kasinathan RS, et al. (2004) Plasmodium induces swelling-activated ClC-2 anion channels in the host erythrocyte. J Biol Chem 279: 41444-41452.

Hudson VM (2001) Rethinking cystic fibrosis pathology: the critical role of abnormal reduced glutathione (GSH) transport caused by CFTR mutation. Free Radic Biol Med 30:1440-1461.

Hussy N (1991) Developmental change in calcium-activated chloride current during the differentiation of Xenopus spinal neurons in culture. Dev Biol 147:225-238.

Hwang TC and Sheppard DN (2009) Gating of the CFTR Cl- channel by ATP-driven nucleotide-binding domain dimerisation. $J$ Physiol 587:2151-2161.

Hyzinski-García MC, Rudkouskaya A, and Mongin AA (2014) LRRC8A protein is indispensable for swelling-activated and ATP-induced release of excitatory amino acids in rat astrocytes. $J$ Physiol 592:4855-4862.

Infield DT, Cui G, Kuang C, and McCarty NA (2016) Positioning of extracellular loop 1 affects pore gating of the cystic fibrosis transmembrane conductance regulator. Am J Physiol Lung Cell Mol Physiol 310:L403-L414.

Inoue $\mathrm{H}$, Mori S, Morishima S, and Okada Y (2005) Volume-sensitive chloride channels in mouse cortical neurons: characterization and role in volume regulation. Eur J Neurosci 21:1648-1658.

Inoue $\mathrm{H}$, Ohtaki $\mathrm{H}$, Nakamachi T, Shioda S, and Okada Y (2007) Anion channel blockers attenuate delayed neuronal cell death induced by transient forebrain ischemia. J Neurosci Res 85:1427-1435.

Inoue $\mathrm{H}$ and Okada $\mathrm{Y}$ (2007) Roles of volume-sensitive chloride channel in excitotoxic neuronal injury. $J$ Neurosci 27:1445-1455.

Islam MR, Merzlyak P, Okada T, Sabirov RZ, and Okada Y (2013) Searching for the molecular basis of maxi-anion channel. J Physiol Sci 63 (Suppl 1):S133.

Islam MR, Uramoto H, Okada T, Sabirov RZ, and Okada Y (2012) Maxi-anion channel and pannexin 1 hemichannel constitute separate pathways for swellinginduced ATP release in murine L929 fibrosarcoma cells. Am J Physiol Cell Physiol 303:C924-C935.

Jackson PS, Morrison R, and Strange K (1994) The volume-sensitive organic osmolyte-anion channel VSOAC is regulated by nonhydrolytic ATP binding. Am $J$ Physiol 267:C1203-C1209.

Jackson PS and Strange K (1995) Characterization of the voltage-dependent properties of a volume-sensitive anion conductance. J Gen Physiol 105:661-676.

Jalonen T (1993) Single-channel characteristics of the large-conductance anion channel in rat cortical astrocytes in primary culture. Glia 9:227-237.

Jalonen T, Johansson S, Holopainen I, Oja SS, and Arhem P (1989) A highconductance multi-state anion channel in cultured rat astrocytes. Acta Physiol Scand 136:611-612.

James AF, Tominaga T, Okada Y, and Tominaga M (1996) Distribution of cAMPactivated chloride current and CFTR mRNA in the guinea pig heart. Circ Res 79: 201-207.

Jentsch TJ, Stein V, Weinreich F, and Zdebik AA (2002) Molecular structure and physiological function of chloride channels. Physiol Rev 82:503-568.

Jiao JD, Xu CQ, Yue P, Dong DL, Li Z, Du ZM, and Yang BF (2006) Volume-sensitive outwardly rectifying chloride channels are involved in oxidative stress-induced apoptosis of mesangial cells. Biochem Biophys Res Commun 340:277-285.

Jordt SE and Jentsch TJ (1997) Molecular dissection of gating in the ClC-2 chloride channel. EMBO J 16:1582-1592.

Jovov B, Ismailov II, Berdiev BK, Fuller CM, Sorscher EJ, Dedman JR, Kaetzel MA and Benos DJ (1995) Interaction between cystic fibrosis transmembrane conductance regulator and outwardly rectified chloride channels. J Biol Chem 270: 29194-29200.

Juul CA, Grubb S, Poulsen KA, Kyed T, Hashem N, Lambert IH, Larsen EH, and Hoffmann EK (2014) Anoctamin 6 differs from VRAC and VSOAC but is involved in apoptosis and supports volume regulation in the presence of $\mathrm{Ca}^{2+}$. Pflugers Arch 466:1899-1910. 
Kajita H, Whitwell C, and Brown PD (2000) Properties of the inward-rectifying $\mathrm{Cl}^{-}$ channel in rat choroid plexus: regulation by intracellular messengers and inhibition by divalent cations. Pflugers Arch 440:933-940.

Kajiya H, Okamoto F, Ohgi K, Nakao A, Fukushima H, and Okabe K (2009) Characteristics of $\mathrm{ClC7} \mathrm{Cl}^{-}$channels and their inhibition in mutant (G215R) associated with autosomal dominant osteopetrosis type II in native osteoclasts and hClcn7 gene-expressing cells. Pflugers Arch 458:1049-1059.

Kanno T and Takishima T (1990) Chloride and potassium channels in U937 human monocytes. J Membr Biol 116:149-161.

Kariya C, Leitner H, Min E, van Heeckeren C, van Heeckeren A, and Day BJ (2007) A role for CFTR in the elevation of glutathione levels in the lung by oral glutathione administration. Am J Physiol Lung Cell Mol Physiol 292: L1590-L1597.

Kartner N, Hanrahan JW, Jensen TJ, Naismith AL, Sun SZ, Ackerley CA, Reyes EF, Tsui LC, Rommens JM, Bear CE, et al. (1991) Expression of the cystic fibrosis gene in non-epithelial invertebrate cells produces a regulated anion conductance. Cell 64:681-691.

Kasuya G, Nakane T, Yokoyama T, Jia Y, Inoue M, Watanabe K, Nakamura R, Nishizawa T, Kusakizako T, Tsutsumi A, et al. (2018) Cryo-EM structures of the human volume-regulated anion channel LRRC8. Nat Struct Mol Biol 25:797-804.

Kawahara K and Takuwa N (1991) Bombesin activates large-conductance chloride channels in Swiss 3T3 fibroblasts. Biochem Biophys Res Commun 177:292-298.

Kefauver JM, Saotome K, Dubin AE, Pallesen J, Cottrell CA, Cahalan SM, Qiu Z, Hong G, Crowley CS, Whitwam T, et al. (2018) Structure of the human volume regulated anion channel. eLife 7:e38461.

Kim Y, Anderson MO, Park J, Lee MG, Namkung W, and Verkman AS (2015) Benzopyrimido-pyrrolo-oxazine-dione (R)-BPO-27 inhibits CFTR chloride channel gating by competition with ATP. Mol Pharmacol 88:689-696.

Kimelberg HK, Goderie SK, Higman S, Pang S, and Waniewski RA (1990) Swellinginduced release of glutamate, aspartate, and taurine from astrocyte cultures. $J$ Neurosci 10:1583-1591.

Kirk K (1997) Swelling-activated organic osmolyte channels. J Membr Biol 158:1-16.

Kirk KL and Wang W (2011) A unified view of cystic fibrosis transmembrane conductance regulator (CFTR) gating: combining the allosterism of a ligand-gated channel with the enzymatic activity of an ATP-binding cassette (ABC) transporter. J Biol Chem 286:12813-12819.

Klausen TK, Bergdahl A, Hougaard C, Christophersen P, Pedersen SF, and Hoffmann EK (2007) Cell cycle-dependent activity of the volume- and $\mathrm{Ca}^{2+}$ activated anion currents in Ehrlich lettre ascites cells. J Cell Physiol 210: 831-842.

Kleene SJ (1993) Origin of the chloride current in olfactory transduction. Neuron 11: $123-132$

Kobe B and Kajava AV (2001) The leucine-rich repeat as a protein recognition motif Curr Opin Struct Biol 11:725-732.

Kogan I, Ramjeesingh M, Li C, Kidd JF, Wang Y, Leslie EM, Cole SP, and Bear CE (2003) CFTR directly mediates nucleotide-regulated glutathione flux. EMBO J 22 $1981-1989$.

Kokubun S, Saigusa A, and Tamura T (1991) Blockade of Cl channels by organic and inorganic blockers in vascular smooth muscle cells. Pflugers Arch 418:204-213.

Kopeikin Z, Sohma Y, Li M, and Hwang TC (2010) On the mechanism of CFTR inhibition by a thiazolidinone derivative. J Gen Physiol 136:659-671.

Kraig RP, Pulsinelli WA, and Plum F (1985) Heterogeneous distribution of hydrogen and bicarbonate ions during complete brain ischemia. Prog Brain Res 63:155-166

Krasilnikov OV, Sabirov RZ, and Okada Y (2011) ATP hydrolysis-dependent asymmetry of the conformation of CFTR channel pore. J Physiol Sci 61: $267-278$.

Kubo M and Okada Y (1992) Volume-regulatory $\mathrm{Cl}^{-}$channel currents in cultured human epithelial cells. J Physiol 456:351-371.

Kucherenko YV, Mörsdorf D, and Lang F (2009) Acid-sensitive outwardly rectifying anion channels in human erythrocytes. $J$ Membr Biol 230:1-10.

Kumagai K, Imai S, Toyoda F, Okumura N, Isoya E, Matsuura H, and Matsusue $\mathrm{Y}$ (2012) 17ß-Oestradiol inhibits doxorubicin-induced apoptosis via block of the volume-sensitive $\mathrm{Cl}(-)$ current in rabbit articular chondrocytes. $\mathrm{Br} \mathrm{J}$ Pharmacol 166:702-720.

Kumar L, Chou J, Yee CS, Borzutzky A, Vollmann EH, von Andrian UH, Park SY, Hollander G, Manis JP, Poliani PL, et al. (2014) Leucine-rich repeat containing 8A (LRRC8A) is essential for T lymphocyte development and function. J Exp Med 211: 929-942.

Kunzelmann K, Kiser GL, Schreiber R, and Riordan JR (1997) Inhibition of epithelial $\mathrm{Na}^{+}$currents by intracellular domains of the cystic fibrosis transmembrane conductance regulator. FEBS Lett 400:341-344.

Kunzelmann K and Schreiber R (1999) CFTR, a regulator of channels. J Membr Biol 168:1-8.

Kunzelmann K, Schreiber R, Kmit A, Jantarajit W, Martins JR, Faria D, Kongsuphol $\mathrm{P}$, Ousingsawat J, and Tian Y (2012) Expression and function of epithelial anoctamins. Exp Physiol 97:184-192.

Kurbannazarova RS, Bessonova SV, Okada Y, and Sabirov RZ (2011) Swellingactivated anion channels are essential for volume regulation of mouse thymocytes. Int J Mol Sci 12:9125-9137.

Kuruma A and Hartzell HC (2000) Bimodal control of a $\mathrm{Ca}(2+)$-activated $\mathrm{Cl}(-)$ channel by different $\mathrm{Ca}(2+)$ signals. $J$ Gen Physiol 115:59-80.

Kürz LL, Klink H, Jakob I, Kuchenbecker M, Benz S, Lehmann-Horn F, and Rüdel R (1999) Identification of three cysteines as targets for the $\mathrm{Zn}^{2+}$ blockade of the human skeletal muscle chloride channel. J Biol Chem 274:11687-11692.

Kürz L, Wagner S, George AL Jr, and Rüdel R (1997) Probing the major skeletal muscle chloride channel with $\mathrm{Zn}^{2+}$ and other sulfhydryl-reactive compounds. Pflugers Arch 433:357-363.

Lambert S and Oberwinkler J (2005) Characterization of a proton-activated, outwardly rectifying anion channel. J Physiol 567:191-213.

Lang F, editor (1998) Cell Volume Regulation, Karger, Basel, Switzerland.
Lansman JB, Hess P, and Tsien RW (1986) Blockade of current through single calcium channels by $\mathrm{Cd}^{2+}, \mathrm{Mg}^{2+}$, and $\mathrm{Ca}^{2+}$. Voltage and concentration dependence of calcium entry into the pore. J Gen Physiol 88:321-347.

Leaney JL, Marsh SJ, and Brown DA (1997) A swelling-activated chloride current in rat sympathetic neurones. J Physiol 501:555-564.

Ledoux J, Greenwood IA, and Leblanc N (2005) Dynamics of $\mathrm{Ca}^{2+}$-dependent $\mathrm{Cl}^{-}$ channel modulation by niflumic acid in rabbit coronary arterial myocytes. $\mathrm{Mol}$ Pharmacol 67:163-173.

Lee CC, Freinkman E, Sabatini DM, and Ploegh HL (2014) The protein synthesis inhibitor blasticidin s enters mammalian cells via leucine-rich repeat-containing protein 8D. J Biol Chem 289:17124-17131.

Lee EL, Shimizu T, Ise T, Numata T, Kohno K, and Okada Y (2007) Impaired activity of volume-sensitive $\mathrm{Cl}^{-}$channel is involved in cisplatin resistance of cancer cells. $J$ Cell Physiol 211:513-521.

Levesque PC, Hart PJ, Hume JR, Kenyon JL, and Horowitz B (1992) Expression of cystic fibrosis transmembrane regulator $\mathrm{Cl}^{-}$channels in heart. Circ Res $\mathbf{7 1}$ 1002-1007.

Levitan I, Almonte C, Mollard P, and Garber SS (1995) Modulation of a volumeregulated chloride current by F-actin. J Membr Biol 147:283-294.

Levitan I and Garber SS (1996) VRAC activation is correlated with membrane cytoskeletal tension. J Gen Physiol 108:25a.

l'Hoste S, Chargui A, Belfodil R, Corcelle E, Duranton C, Rubera I, Poujeol C, Mograbi B, Tauc M, and Poujeol P (2010) CFTR mediates apoptotic volume decrease and cell death by controlling glutathione efflux and ROS production in cultured mice proximal tubules. Am J Physiol Renal Physiol 298 F435-F453.

Light DB, Schwiebert EM, Fejes-Toth G, Naray-Fejes-Toth A, Karlson KH, McCann FV, and Stanton BA (1990) Chloride channels in the apical membrane of cortical collecting duct cells. Am J Physiol 258:F273-F280.

Linsdell P (2000) Inhibition of cystic fibrosis transmembrane conductance regulator chloride channel currents by arachidonic acid. Can J Physiol Pharmacol 78: 490-499.

Linsdell P (2005) Location of a common inhibitor binding site in the cytoplasmic vestibule of the cystic fibrosis transmembrane conductance regulator chloride channel pore. J Biol Chem 280:8945-8950.

Linsdell P (2014) Cystic fibrosis transmembrane conductance regulator chloride channel blockers: pharmacological, biophysical and physiological relevance. World J Biol Chem 5:26-39.

Linsdell P and Hanrahan JW (1996) Disulphonic stilbene block of cystic fibrosis transmembrane conductance regulator $\mathrm{Cl}^{-}$channels expressed in a mammalian cell line and its regulation by a critical pore residue. J Physiol 496:687-693.

Linsdell P and Hanrahan JW (1998) Adenosine triphosphate-dependent asymmetry of anion permeation in the cystic fibrosis transmembrane conductance regulator chloride channel. J Gen Physiol 111:601-614.

Linsdell P, Tabcharani JA, Rommens JM, Hou YX, Chang XB, Tsui LC, Riordan JR and Hanrahan JW (1997) Permeability of wild-type and mutant cystic fibrosis transmembrane conductance regulator chloride channels to polyatomic anions. $J$ Gen Physiol 110:355-364.

Lipecka J, Bali M, Thomas A, Fanen P, Edelman A, and Fritsch J (2002) Distribution of ClC-2 chloride channel in rat and human epithelial tissues. Am J Physiol Cell Physiol 282:C805-C816.

Liu F, Zhang Z, Csanády L, Gadsby DC, and Chen J (2017) Molecular structure of the human CFTR ion channel. Cell 169:85-95.e8.

Liu HT, Akita T, Shimizu T, Sabirov RZ, and Okada Y (2009) Bradykinin-induced astrocyte-neuron signalling: glutamate release is mediated by ROS-activated volume-sensitive outwardly rectifying anion channels. $J$ Physiol 587:2197-2209.

Liu HT, Sabirov RZ, and Okada Y (2008a) Oxygen-glucose deprivation induce ATP release via maxi-anion channels in astrocytes. Purinergic Signal 4: $147-154$

Liu HT, Tashmukhamedov BA, Inoue H, Okada Y, and Sabirov RZ (2006) Roles of two types of anion channels in glutamate release from mouse astrocytes under ischemic or osmotic stress. Glia 54:343-357.

Liu HT, Toychiev AH, Takahashi N, Sabirov RZ, and Okada Y (2008b) Maxi-anion channel as a candidate pathway for osmosensitive ATP release from mouse astrocytes in primary culture. Cell Res 18:558-565.

Liu Y, Oiki S, Tsumura T, Shimizu T, and Okada Y (1998) Glibenclamide blocks volume-sensitive $\mathrm{Cl}^{-}$channels by dual mechanisms. Am J Physiol 275:C343-C351. Liu Y, Zhang H, Huang D, Qi J, Xu J, Gao H, Du X, Gamper N, and Zhang H (2015) Characterization of the effects of $\mathrm{Cl}^{-}$channel modulators on TMEM16A and bestrophin-1 $\mathrm{Ca}^{2+}$ activated $\mathrm{Cl}^{-}$channels. Pflugers Arch 467:1417-1430.

Loewen ME and Forsyth GW (2005) Structure and function of CLCA proteins. Physiol Rev 85:1061-1092.

Lutter D, Ullrich F, Lueck JC, Kempa S, and Jentsch TJ (2017) Selective transport of neurotransmitters and modulators by distinct volume-regulated LRRC8 anion channels. J Cell Sci 130:1122-1133.

Ma T, Thiagarajah JR, Yang H, Sonawane ND, Folli C, Galietta LJ, and Verkman AS (2002) Thiazolidinone CFTR inhibitor identified by high-throughput screening blocks cholera toxin-induced intestinal fluid secretion. J Clin Invest 110:1651-1658.

Maeno E, Ishizaki Y, Kanaseki T, Hazama A, and Okada Y (2000) Normotonic cell shrinkage because of disordered volume regulation is an early prerequisite to apoptosis. Proc Natl Acad Sci USA 97:9487-9492.

Maertens C, Wei L, Droogmans G, and Nilius B (2000) Inhibition of volume-regulated and calcium-activated chloride channels by the antimalarial mefloquine. $J$ Pharmacol Exp Ther 295:29-36.

Malinowska DH, Kupert EY, Bahinski A, Sherry AM, and Cuppoletti J (1995) Cloning, functional expression, and characterization of a PKA-activated gastric $\mathrm{Cl}^{-}$ channel. Am J Physiol 268:C191-C200.

Malvezzi M, Chalat M, Janjusevic R, Picollo A, Terashima H, Menon AK, and Accardi A (2013) $\mathrm{Ca}^{2+}$-dependent phospholipid scrambling by a reconstituted TMEM16 ion channel. Nat Commun 4:2367. 
Manabe K, Shimizu T, Morishima S, and Okada Y (2004) Regulatory volume increase after secretory volume decrease in colonic epithelial cells under muscarinic stimulation. Pflugers Arch 448:596-604.

Mao J, Xu B, Li H, Chen L, Jin X, Zhu J, Wang W, Zhu L, Zuo W, Chen W, et al. (2011) Lack of association between stretch-activated and volume-activated $\mathrm{Cl}^{-}$ currents in hepatocellular carcinoma cells. J Cell Physiol 226:1176-1185.

Marmorstein LY, Wu J, McLaughlin P, Yocom J, Karl MO, Neussert R, Wimmers S, Stanton JB, Gregg RG, Strauss O, et al. (2006) The light peak of the electroretinogram is dependent on voltage-gated calcium channels and antagonized by bestrophin (best-1). J Gen Physiol 127:577-589.

Marquardt A, Stöhr H, Passmore LA, Krämer F, Rivera A, and Weber BH (1998) Mutations in a novel gene, VMD2, encoding a protein of unknown properties cause juvenileonset vitelliform macular dystrophy (Best's disease). Hum Mol Genet 7:1517-1525.

Marunaka Y (1997) Hormonal and osmotic regulation of $\mathrm{NaCl}$ transport in renal distal nephron epithelium. Jpn J Physiol 47:499-511.

Matchkov VV, Larsen P, Bouzinova EV, Rojek A, Boedtkjer DM, Golubinskaya V, Pedersen FS, Aalkjaer C, and Nilsson H (2008) Bestrophin-3 (vitelliform macular dystrophy 2-like 3 protein) is essential for the cGMP-dependent calcium-activated chloride conductance in vascular smooth muscle cells. Circ Res 103:864-872.

Matsuda JJ, Filali MS, Collins MM, Volk KA, and Lamb FS (2010a) The ClC-3 Cl-/ $/ \mathrm{H}^{+}$ antiporter becomes uncoupled at low extracellular $\mathrm{pH}$. J Biol Chem 285 2569-2579.

Matsuda JJ, Filali MS, Moreland JG, Miller FJ, and Lamb FS (2010b) Activation of swelling-activated chloride current by tumor necrosis factor-alpha requires ClC-3dependent endosomal reactive oxygen production. $J$ Biol Chem 285:22864-22873.

McCann FV, McCarthy DC, Keller TM, and Noelle RJ (1989) Characterization of a large conductance non-selective anion channel in B lymphocytes. Cell Signal 1 31-44.

McCarty NA (2000) Permeation through the CFTR chloride channel. J Exp Biol 203: 1947-1962.

McCarty NA, McDonough S, Cohen BN, Riordan JR, Davidson N, and Lester HA (1993) Voltage-dependent block of the cystic fibrosis transmembrane conductance regulator $\mathrm{Cl}^{-}$channel by two closely related arylaminobenzoates. J Gen Physiol 102:1-23.

McGill JM, Basavappa S, and Fitz JG (1992) Characterization of high-conductance anion channels in rat bile duct epithelial cells. Am J Physiol 262:G703-G710.

McGill JM, Gettys TW, Basavappa S, and Fitz JG (1993) GTP-binding proteins regulate high conductance anion channels in rat bile duct epithelial cells. J Membr Biol 133:253-261.

McLarnon JG and Kim SU (1991) Ion channels in cultured adult human Schwann cells. Glia 4:534-539.

McNicholas CM, Guggino WB, Schwiebert EM, Hebert SC, Giebisch G, and Egan ME (1996) Sensitivity of a renal $\mathrm{K}^{+}$channel (ROMK2) to the inhibitory sulfonylurea compound glibenclamide is enhanced by coexpression with the ATP-binding cassette transporter cystic fibrosis transmembrane regulator. Proc Natl Acad Sci USA 93:8083-8088.

McNicholas CM, Nason MW Jr, Guggino WB, Schwiebert EM, Hebert SC, Giebisch G, and Egan ME (1997) A functional CFTR-NBF1 is required for ROMK2-CFTR interaction. Am J Physiol 273:F843-F848.

Melis N, Tauc M, Cougnon M, Bendahhou S, Giuliano S, Rubera I, and Duranton C (2014) Revisiting CFTR inhibition: a comparative study of CFTRinh -172 and GlyH-101 inhibitors. Br J Pharmacol 171:3716-3727.

Meyer K and Korbmacher C (1996) Cell swelling activates ATP-dependent voltagegated chloride channels in M-1 mouse cortical collecting duct cells. J Gen Physiol 108:177-193.

Miledi R (1982) A calcium-dependent transient outward current in Xenopus laevis oocytes. Proc R Soc Lond B Biol Sci 215:491-497.

Milenkovic A, Brandl C, Milenkovic VM, Jendryke T, Sirianant L, Wanitchakool P, Zimmermann S, Reiff CM, Horling F, Schrewe H, et al. (2015) Bestrophin 1 is indispensable for volume regulation in human retinal pigment epithelium cells. Proc Natl Acad Sci USA 112:E2630-E2639.

Minor DL Jr (2017) Channel surfing uncovers a dual-use transporter. EMBO J 36: $3272-3273$.

Mitchell CH, Wang L, and Jacob TJC (1997a) A large-conductance chloride channel in pigmented ciliary epithelial cells activated by GTPgammaS. J Membr Biol 158: $167-175$

Mitchell CH, Zhang JJ, Wang L, and Jacob TJ (1997b) Volume-sensitive chloride current in pigmented ciliary epithelial cells: role of phospholipases. Am J Physiol 272:C212-C222.

Miwa A, Ueda K, and Okada Y (1997) Protein kinase C-independent correlation between P-glycoprotein expression and volume sensitivity of $\mathrm{Cl}^{-}$channel. $\mathrm{J} \mathrm{Membr}$ Biol 157:63-69.

Miyata H, Kinosita K Jr, and Marriott G (1997) Cooperative association of actin protomers and crosslinked actin oligomers in filaments at low ionic strength. $J$ Biochem 121:527-533.

Morán J, Sabanero M, Meza I, and Pasantes-Morales H (1996) Changes of actin cytoskeleton during swelling and regulatory volume decrease in cultured astrocytes. Am J Physiol 271:C1901-C1907.

Morise M, Ito Y, Matsuno T, Hibino Y, Mizutani T, Ito S, Hashimoto N, Kondo M, Imaizumi K, and Hasegawa Y (2010) Heterologous regulation of anion transporters by menthol in human airway epithelial cells. Eur J Pharmacol 635:204-211.

Morishima S, Shimizu T, Kida H, and Okada Y (2000) Volume expansion sensitivity of swelling-activated $\mathrm{Cl}(-)$ channel in human epithelial cells. Jpn $J$ Physiol 50: $277-280$

Muanprasat C, Sonawane ND, Salinas D, Taddei A, Galietta LJ, and Verkman AS (2004) Discovery of glycine hydrazide pore-occluding CFTR inhibitors: mechanism, structure-activity analysis, and in vivo efficacy. J Gen Physiol 124:125-137.

Murray CB, Morales MM, Flotte TR, McGrath-Morrow SA, Guggino WB, and Zeitlin PL (1995) CIC-2: a developmentally dependent chloride channel expressed in the fetal lung and downregulated after birth. Am J Respir Cell Mol Biol 12:597-604.
Namkung W, Phuan PW, and Verkman AS (2011) TMEM16A inhibitors reveal TMEM16A as a minor component of calcium-activated chloride channel conductance in airway and intestinal epithelial cells. $J$ Biol Chem 286 2365-2374.

Namkung W, Thiagarajah JR, Phuan PW, and Verkman AS (2010) Inhibition of $\mathrm{Ca}^{2+}$-activated $\mathrm{Cl}^{-}$channels by gallotannins as a possible molecular basis for health benefits of red wine and green tea. FASEB $J$ 24:4178-4186.

Nehrke K, Arreola J, Nguyen HV, Pilato J, Richardson L, Okunade G, Baggs R, Shull GE, and Melvin JE (2002) Loss of hyperpolarization-activated Cl(-) current in salivary acinar cells from Clcn2 knockout mice. J Biol Chem 277:23604-23611.

Neussert R, Müller C, Milenkovic VM, and Strauss O (2010) The presence of bestrophin-1 modulates the $\mathrm{Ca}^{2+}$ recruitment from $\mathrm{Ca}^{2+}$ stores in the ER. Pflugers Arch 460:163-175

Niisato N, Ito Y, and Marunaka Y (1999) Activation of $\mathrm{Cl}^{-}$channel and $\mathrm{Na}^{+} / \mathrm{K}^{+} / 2 \mathrm{Cl}$ cotransporter in renal epithelial A6 cells by flavonoids: genistein, daidzein, and apigenin. Biochem Biophys Res Commun 254:368-371.

Nilius B and Droogmans G (2001) Ion channels and their functional role in vascular endothelium. Physiol Rev 81:1415-1459.

Nilius B, Eggermont J, Voets T, Buyse G, Manolopoulos V, and Droogmans G (1997a) Properties of volume-regulated anion channels in mammalian cells. Prog Biophys Mol Biol 68:69-119.

Nilius B, Oike M, Zahradnik I, and Droogmans G (1994a) Activation of a $\mathrm{Cl}^{-}$current by hypotonic volume increase in human endothelial cells. J Gen Physiol 103:787-805.

Nilius B, Prenen J, Szücs G, Wei L, Tanzi F, Voets T, and Droogmans G (1997b) Calcium-activated chloride channels in bovine pulmonary artery endothelial cells. J Physiol 498:381-396

Nilius B, Prenen J, Voets T, Eggermont J, and Droogmans G (1998) Activation of volume-regulated chloride currents by reduction of intracellular ionic strength in bovine endothelial cells. $J$ Physiol 506:353-361.

Nilius B, Sehrer J, and Droogmans G (1994b) Permeation properties and modulation of volume-activated $\mathrm{Cl}(-)$-currents in human endothelial cells. $\mathrm{Br} J$ Pharmacol 112 : 1049-1056

Nobles M, Higgins CF, and Sardini A (2004) Extracellular acidification elicits a chloride current that shares characteristics with $\mathrm{I}_{\mathrm{Cl}(\text { swell) }}$ Am J Physiol Cell Physiol 287:C1426-C1435.

Numata T and Okada Y (2009) Expression of TRPM7 channels switches acidosisinduced cell death from apoptosis to necrosis in human epithelial cells. $J$ Physiol Sci 59 (Suppl 1):153.

Numata T, Sato-Numata K, Okada Y, and Inoue R (2018) Cellular mechanism for herbal medicine Junchoto to facilitate intestinal $\mathrm{Cl}^{-} /$water secretion that involves cAMP-dependent activation of CFTR. J Nat Med 72:694-705.

O'Donnell EK, Sedlacek RL, Singh AK, and Schultz BD (2000) Inhibition of enterotoxin-induced porcine colonic secretion by diarylsulfonylureas in vitro. Am $J$ Physiol Gastrointest Liver Physiol 279:G1104-G1112.

O'Donnell MJ, Kelly SP, Nurse CA, and Wood CM (2001) A maxi $\mathrm{Cl}^{-}$channel in cultured pavement cells from the gills of the freshwater rainbow trout Oncorhynchus mykiss. $J$ Exp Biol 204:1783-1794.

O'Driscoll KE, Hatton WJ, Burkin HR, Leblanc N, and Britton FC (2008) Expression, localization, and functional properties of bestrophin 3 channel isolated from mouse heart. Am J Physiol Cell Physiol 295:C1610-C1624.

Oh SJ, Hwang SJ, Jung J, Yu K, Kim J, Choi JY, Hartzell HC, Roh EJ, and Lee CJ (2013) MONNA, a potent and selective blocker for transmembrane protein with unknown function 16/anoctamin-1. Mol Pharmacol 84:726-735.

Oh U and Jung J (2016) Cellular functions of TMEM16/anoctamin. Pflugers Arch 468:443-453.

Ohana E, Yang D, Shcheynikov N, and Muallem S (2009) Diverse transport modes by the solute carrier 26 family of anion transporters. $J$ Physiol 587:2179-2185.

Ohgi K, Okamoto F, Kajiya H, Sakagami R, and Okabe K (2011) Antibodies against ClC7 inhibit extracellular acidification-induced $\mathrm{Cl}^{-}$currents and bone resorption activity in mouse osteoclasts. Naunyn Schmiedebergs Arch Pharmacol 383:79-90.

Oiki S, Kubo M, and Okada Y (1994) $\mathrm{Mg}^{2+}$ and ATP-dependence of volume-sensitive $\mathrm{Cl}^{-}$channels in human epithelial cells. Jpn J Physiol 44 (Suppl 2):S77-S79.

Okada T, Akita T, Sato-Numata K, Islam MR, and Okada Y (2014) A newly cloned ClC-3 isoform, ClC-3d, as well as ClC-3a mediates Cd-sensitive outwardly rectifying anion currents. Cell Physiol Biochem 33:539-556.

Okada T, Islam MR, Tsiferova NA, Okada Y, and Sabirov RZ (2017) Specific and essential but not sufficient roles of LRRC8A in the activity of volumesensitive outwardly rectifying anion channel (VSOR). Channels (Austin) 11 109-120.

Okada Y (1997) Volume expansion-sensing outward-rectifier $\mathrm{Cl}^{-}$channel: fresh start to the molecular identity and volume sensor. Am J Physiol 273:C755-C789.

Okada Y (1998a) Cell volume-sensitive chloride channels. Contrib Nephrol 123: 21-33.

Okada Y, editor (1998b) Cell Volume Regulation: The Molecular Mechanism and Volume Sensing Machinery, Elsevier, Amsterdam.

Okada Y (2004) Ion channels and transporters involved in cell volume regulation and sensor mechanisms. Cell Biochem Biophys 41:233-258.

Okada Y (2006) Cell volume-sensitive chloride channels: phenotypic properties and molecular identity. Contrib Nephrol 152:9-24.

Okada Y and Hazama A (1989) Volume-regulatory ion channels in epithelial cells. News Physiol Sci 4:238-242.

Okada Y, Hazama A, Hashimoto A, Maruyama Y, and Kubo M (1992) Exocytosis upon osmotic swelling in human epithelial cells. Biochim Biophys Acta 1107: 201-205.

Okada Y, Kubo M, Oiki S, Petersen CCH, Tominaga M, Hazama A, and Morishima S (1994a) Properties of volume-sensitive $\mathrm{Cl}^{-}$channels in a human epithelial cell line. Jpn J Physiol 44 (Suppl 2):S31-S35.

Okada Y, Maeno E, Shimizu T, Dezaki K, Wang J, and Morishima S (2001) Receptormediated control of regulatory volume decrease (RVD) and apoptotic volume decrease (AVD). J Physiol 532:3-16. 
Okada Y, Maeno E, Shimizu T, Manabe K, Mori S, and Nabekura T (2004) Dual roles of plasmalemmal chloride channels in induction of cell death. Pflugers Arch 448: 287-295.

Okada Y, Oiki S, Hazama A, and Morishima S (1998) Criteria for the molecular identification of the volume-sensitive outwardly rectifying $\mathrm{Cl}^{-}$channel. $J$ Gen Physiol 112:365-367.

Okada Y, Okada T, Islam MR, and Sabirov RZ (2018) Molecular identities and ATP release activities of two types of volume-regulatory anion channels, VSOR and Maxi-Cl. Curr Top Membr 81:125-176.

Okada Y, Petersen CCH, Kubo M, Morishima S, and Tominaga M (1994b) Osmotic swelling activates intermediate-conductance $\mathrm{Cl}^{-}$channels in human intestinal epithelial cells. Jpn J Physiol 44:403-409.

Okada Y, Sato K, and Numata T (2009a) Pathophysiology and puzzles of the volumesensitive outwardly rectifying anion channel. J Physiol 587:2141-2149.

Okada Y, Sato K, Toychiev AH, Suzuki M, Dutta AK, Inoue H, and Sabirov RZ (2009b) The puzzles of volume-activated anion channels, in Physiology and Pathology of Chloride Transporters and Channels in the Nervous System: From Molecules to Disease (Alvarez-Leefmans FJ and Delpire E eds) pp 283-306, Elsevier, San Diego, CA.

Olesen SP and Bundgaard M (1992) Chloride-selective channels of large conductance in bovine aortic endothelial cells. Acta Physiol Scand 144:191-198.

Pahapill PA and Schlichter LC (1992) $\mathrm{Cl}^{-}$channels in intact human T lymphocytes. $J$ Membr Biol 125:171-183.

Pangrsic T, Potokar M, Haydon PG, Zorec R, and Kreft M (2006) Astrocyte swelling leads to membrane unfolding, not membrane insertion. $J$ Neurochem 99:514-523.

Park H, Oh SJ, Han KS, Woo DH, Park H, Mannaioni G, Traynelis SF, and Lee CJ (2009) Bestrophin-1 encodes for the $\mathrm{Ca}^{2+}$-activated anion channel in hippocampal astrocytes. $J$ Neurosci 29:13063-13073.

Park K, Arreola J, Begenisich T, and Melvin JE (1998) Comparison of voltageactivated $\mathrm{Cl}^{-}$channels in rat parotid acinar cells with ClC-2 in a mammalian expression system. J Membr Biol 163:87-95.

Parkerson KA and Sontheimer H (2004) Biophysical and pharmacological characterization of hypotonically activated chloride currents in cortical astrocytes. Glia 46:419-436.

Payne JA (2009) The potassium-chloride cotransporters: from cloning to structure and function, in Physiology and Pathology of Chloride Transporters and Channels in the Nervous System: From Molecules to Diseases (Alvarez-Leefmans FJ and Delpire E eds) pp 333-356, Elsevier, San Diego, CA.

Pedemonte N and Galietta LJ (2014) Structure and function of TMEM16 proteins (anoctamins). Physiol Rev 94:419-459.

Pedersen SF, Hoffmann EK, and Mills JW (2001) The cytoskeleton and cell volume regulation. Comp Biochem Physiol A Mol Integr Physiol 130:385-399.

Pedersen SF, Klausen TK, and Nilius B (2015) The identification of a volumeregulated anion channel: an amazing Odyssey. Acta Physiol (Oxf) 213:868-881.

Pedersen SF, Mills JW, and Hoffmann EK (1999) Role of the F-actin cytoskeleton in the RVD and RVI processes in Ehrlich ascites tumor cells. Exp Cell Res 252 $63-74$

Pedersen SF, Okada Y, and Nilius B (2016) Biophysics and physiology of the volumeregulated anion channel (VRAC)/volume-sensitive outwardly rectifying anion channel (VSOR). Pflugers Arch 468:371-383.

Petersen CCH, Kubo M, Morishima S, Tominaga M, and Okada Y (1994) Singlechannel recordings of volume-sensitive $\mathrm{Cl}^{-}$channels in human intestinal epithelial cells. Jpn J Physiol 44 (Suppl 2):S73-S75.

Petrukhin K, Koisti MJ, Bakall B, Li W, Xie G, Marknell T, Sandgren O, Forsman K, Holmgren G, Andreasson S, et al. (1998) Identification of the gene responsible for Best macular dystrophy. Nat Genet 19:241-247.

Picollo A, Malvezzi M, and Accardi A (2015) TMEM16 proteins: unknown structure and confusing functions. J Mol Biol 427:94-105.

Picollo A and Pusch M (2005) Chloride/proton antiporter activity of mammalian CLC proteins ClC-4 and ClC-5. Nature 436:420-423.

Pifferi S, Dibattista M, and Menini A (2009) TMEM16B induces chloride currents activated by calcium in mammalian cells. Pflugers Arch 458:1023-1038.

Pifferi S, Pascarella G, Boccaccio A, Mazzatenta A, Gustincich S, Menini A, and Zucchelli S (2006) Bestrophin-2 is a candidate calcium-activated chloride channel involved in olfactory transduction. Proc Natl Acad Sci USA 103: 12929-12934.

Piper AS and Large WA (2003) Multiple conductance states of single $\mathrm{Ca}^{2+}$-activated $\mathrm{Cl}^{-}$channels in rabbit pulmonary artery smooth muscle cells. $J$ Physiol 547 181-196.

Planells-Cases R, Lutter D, Guyader C, Gerhards NM, Ullrich F, Elger DA, Kucukosmanoglu A, Xu G, Voss FK, Reincke SM, et al. (2015) Subunit composition of VRAC channels determines substrate specificity and cellular resistance to Pt-based anti-cancer drugs. EMBO J 34:2993-3008.

Platt CD, Chou J, Houlihan P, Badran YR, Kumar L, Bainter W, Poliani PL, Perez CJ, Dent SYR, Clapham DE, et al. (2017) Leucine-rich repeat containing 8A (LRRC8A)-dependent volume-regulated anion channel activity is dispensable for T-cell development and function. J Allergy Clin Immunol 140:1651-1659.e1.

Poletto Chaves LA and Varanda WA (2008) Volume-activated chloride channels in mice Leydig cells. Pflugers Arch 457:493-504.

Pritchard S and Guilak F (2004) The role of F-actin in hypo-osmotically induced cell volume change and calcium signaling in anulus fibrosus cells. Ann Biomed Eng $\mathbf{3 2}$ 103-111.

Pusch M and Jentsch TJ (1994) Molecular physiology of voltage-gated chloride channels. Physiol Rev 74:813-827.

Qiu Z, Dubin AE, Mathur J, Tu B, Reddy K, Miraglia LJ, Reinhardt J, Orth AP, and Patapoutian A (2014) SWELL1, a plasma membrane protein, is an essentia component of volume-regulated anion channel. Cell 157:447-458.

Qu Z and Hartzell HC (2001) Functional geometry of the permeation pathway of $\mathrm{Ca}^{2+}$-activated $\mathrm{Cl}^{-}$channels inferred from analysis of voltage-dependent block. $J$ Biol Chem 276:18423-18429.
$\mathrm{Qu} \mathrm{Z}$, Wei RW, and Hartzell $\mathrm{HC}$ (2003a) Characterization of $\mathrm{Ca}^{2+}$-activated $\mathrm{Cl}^{-}$ currents in mouse kidney inner medullary collecting duct cells. Am J Physiol Renal Physiol 285:F326-F335.

Qu Z, Wei RW, Mann W, and Hartzell HC (2003b) Two bestrophins cloned from Xenopus laevis oocytes express $\mathrm{Ca}(2+)$-activated $\mathrm{Cl}(-)$ currents. J Biol Chem 278 : 49563-49572.

Quasthoff S, Strupp M, and Grafe P (1992) High conductance anion channel in Schwann cell vesicles from rat spinal roots. Glia 5:17-24.

Rabe A, Disser J, and Frömter E (1995) $\mathrm{Cl}^{-}$channel inhibition by glibenclamide is not specific for the CFTR-type $\mathrm{Cl}^{-}$channel. Pflugers Arch 429:659-662.

Raucci FJ Jr, Wijesinghe DS, Chalfant CE, and Baumgarten CM (2010) Exogenous and endogenous ceramides elicit volume-sensitive chloride current in ventricular myocytes. Cardiovasc Res 86:55-62.

Rehncrona S (1985) Brain acidosis. Ann Emerg Med 14:770-776.

Ren Z, Raucci FJ Jr, Browe DM, and Baumgarten CM (2008) Regulation of swellingactivated $\mathrm{Cl}(-)$ current by angiotensin II signalling and NADPH oxidase in rabbit ventricle. Cardiovasc Res 77:73-80.

Riordan JR, Rommens JM, Kerem B, Alon N, Rozmahel R, Grzelczak Z, Zielenski J, Lok S, Plavsic N, Chou JL, et al. (1989) Identification of the cystic fibrosis gene: cloning and characterization of complementary DNA. Science $\mathbf{2 4 5}$ : 1066-1073.

Riquelme G, Llanos P, Tischner E, Neil J, and Campos B (2004) Annexin 6 modulates the maxi-chloride channel of the apical membrane of syncytiotrophoblast isolated from human placenta. $J$ Biol Chem 279:50601-50608.

Riquelme G and Parra M (1999) Regulation of human placental chloride channel by arachidonic acid and other cis unsaturated fatty acids. Am J Obstet Gynecol 180 $469-475$

Riquelme G, Stutzin A, Barros LF, and Liberona JL (1995) A chloride channel from human placenta reconstituted into giant liposomes. Am $J$ Obstet Gynecol 173: $733-738$.

Robertson BE (1998) Inhibition of calcium-activated chloride channels by niflumic acid dilates rat cerebral arteries. Acta Physiol Scand 163:417-418.

Roman RM, Smith RL, Feranchak AP, Clayton GH, Doctor RB, and Fitz JG (2001) ClC-2 chloride channels contribute to HTC cell volume homeostasis. Am J Physiol Gastrointest Liver Physiol 280:G344-G353.

Romanenko VG, Catalán MA, Brown DA, Putzier I, Hartzell HC, Marmorstein AD, Gonzalez-Begne M, Rock JR, Harfe BD, and Melvin JE (2010) Tmem16A encodes the $\mathrm{Ca}^{2+}$-activated $\mathrm{Cl}^{-}$channel in mouse submandibular salivary gland acinar cells. J Biol Chem 285:12990-13001.

Rosenthal R, Bakall B, Kinnick T, Peachey N, Wimmers S, Wadelius C, Marmorstein A, and Strauss O (2006) Expression of bestrophin-1, the product of the VMD2 gene, modulates voltage-dependent $\mathrm{Ca}^{2+}$ channels in retinal pigment epithelial cells. FASEB J 20:178-180.

Rubera I, Barrière H, Tauc M, Bidet M, Verheecke-Mauze C, Poujeol C, Cuiller B, and Poujeol P (2001) Extracellular adenosine modulates a volume-sensitive-like chloride conductance in immortalized rabbit DC1 cells. Am J Physiol Renal Physiol 280:F126-F145.

Russell JM (2000) Sodium-potassium-chloride cotransport. Physiol Rev 80:211-276.

Rutledge E, Denton J, and Strange K (2002) Cell cycle- and swelling-induced activation of a Caenorhabditis elegans ClC channel is mediated by CeGLC-7alpha/beta phosphatases. J Cell Biol 158:435-444.

Sabirov RZ, Dutta AK, and Okada Y (2001) Volume-dependent ATP-conductive largeconductance anion channel as a pathway for swelling-induced ATP release. J Gen Physiol 118:251-266.

Sabirov RZ and Merzlyak PG (2012) Plasmalemmal VDAC controversies and maxianion channel puzzle. Biochim Biophys Acta 1818:1570-1580.

Sabirov RZ, Merzlyak PG, Islam MR, Okada T, and Okada Y (2016) The properties, functions, and pathophysiology of maxi-anion channels. Pflugers Arch 468: $405-420$.

Sabirov RZ, Merzlyak PG, Okada T, Islam MR, Uramoto H, Mori T, Makino Y, Matsuura H, Xie Y, and Okada Y (2017) The organic anion transporter SLCO2A1 constitutes the core component of the Maxi-Cl channel. EMBO $J$ 36:3309-3324.

Sabirov RZ and Okada Y (2004a) ATP-conducting maxi-anion channel: a new player in stress-sensory transduction. Jpn J Physiol 54:7-14.

Sabirov RZ and Okada Y (2004b) Wide nanoscopic pore of maxi-anion channel suits its function as an ATP-conductive pathway. Biophys $J$ 87:1672-1685.

Sabirov RZ and Okada Y (2005) ATP release via anion channels. Purinergic Signal 1: $311-328$.

Sabirov RZ and Okada Y (2009) The maxi-anion channel: a classical channel playing novel roles through an unidentified molecular entity. J Physiol Sci 59:3-21.

Sabirov RZ, Prenen J, Tomita T, Droogmans G, and Nilius B (2000) Reduction of ionic strength activates single volume-regulated anion channels (VRAC) in endothelial cells. Pflugers Arch 439:315-320.

Sabirov RZ, Sheiko T, Liu H, Deng D, Okada Y, and Craigen WJ (2006) Genetic demonstration that the plasma membrane maxianion channel and voltagedependent anion channels are unrelated proteins. J Biol Chem 281:1897-1904.

Saigusa A and Kokubun S (1988) Protein kinase C may regulate resting anion conductance in vascular smooth muscle cells. Biochem Biophys Res Commun $\mathbf{1 5 5}$ 882-889.

Sakai H, Kumano E, Ikari S, and Takeguchi N (1995) A gastric housekeeping $\mathrm{Cl}^{-}$ channel activated via prostaglandin EP3 receptor-mediated $\mathrm{Ca}^{2+} /$ nitric oxide/cGMP pathway. J Biol Chem 270:18781-18785.

Sakai H, Okada Y, Morii M, and Takeguchi N (1989) Anion and cation channels in the basolateral membrane of rabbit parietal cells. Pflugers Arch 414:185-192.

Sala-Rabanal M, Yurtsever Z, Nichols CG, and Brett TJ (2015) Secreted CLCA1 modulates TMEM16A to activate $\mathrm{Ca}(2+)$-dependent chloride currents in human cells. eLife 4:e05875.

Sato K, Numata T, Lee E, and Okada Y (2012) Exploration of the molecular identity of acid- sensitive outwardly rectifying $\mathrm{Cl}^{-}$channel (ASOR). J Physiol Sci 62 (Suppl 1):S84. 
Sato K, Numata T, Saito T, Ueta Y, and Okada Y (2011) $\mathrm{V}_{2}$ receptor-mediated autocrine role of somatodendritic release of AVP in rat vasopressin neurons under hypo-osmotic conditions. Sci Signal 4:ra5.

Sato-Numata K, Numata T, Inoue R, and Okada Y (2016) Distinct pharmacological and molecular properties of the acid-sensitive outwardly rectifying (ASOR) anion channel from those of the volume-sensitive outwardly rectifying (VSOR) anion channel. Pflugers Arch 468:795-803.

Sato-Numata K, Numata T, Inoue R, Sabirov RZ, and Okada Y (2017) Distinct contributions of LRRC8A and its paralogs to the VSOR anion channel from those of the ASOR anion channel. Channels (Austin) 11:167-172.

Sato-Numata K, Numata T, and Okada Y (2014) Temperature sensitivity of acidsensitive outwardly rectifying (ASOR) anion channels in cortical neurons is involved in hypothermic neuroprotection against acidotoxic necrosis. Channels (Austin) 8:278-283.

Sato-Numata K, Numata T, Okada T, and Okada Y (2013) Acid-sensitive outwardly rectifying (ASOR) anion channels in human epithelial cells are highly sensitive to temperature and independent of ClC-3. Pflugers Arch 465:1535-1543.

Sauter DRP, Novak I, Pedersen SF, Larsen EH, and Hoffmann EK (2015) ANO1 (TMEM16A) in pancreatic ductal adenocarcinoma (PDAC). Pflugers Arch 467: $1495-1508$.

Sawada A, Takihara Y, Kim JY, Matsuda-Hashii Y, Tokimasa S, Fujisaki H, Kubota K, Endo H, Onodera T, Ohta H, et al. (2003) A congenital mutation of the novel gene LRRC8 causes agammaglobulinemia in humans. $J$ Clin Invest 112 $1707-1713$.

Scheel O, Zdebik AA, Lourdel S, and Jentsch TJ (2005) Voltage-dependent electrogenic chloride/proton exchange by endosomal CLC proteins. Nature 436:424-427.

Schlichter LC, Grygorczyk R, Pahapill PA, and Grygorczyk C (1990) A large, multiple-conductance chloride channel in normal human T lymphocytes. Pflugers Arch 416:413-421.

Schneider GT, Cook DI, Gage PW, and Young JA (1985) Voltage sensitive, highconductance chloride channels in the luminal membrane of cultured pulmonary alveolar (type II) cells. Pflugers Arch 404:354-357.

Schober AL, Wilson CS, and Mongin AA (2017) Molecular composition and heterogeneity of the LRRC8-containing swelling-activated osmolyte channels in primary rat astrocytes. J Physiol 595:6939-6951.

Schreiber R, Hopf A, Mall M, Greger R, and Kunzelmann K (1999) The firstnucleotide binding domain of the cystic-fibrosis transmembrane conductance regulator is important for inhibition of the epithelial $\mathrm{Na}^{+}$channel. Proc Natl Acad Sci USA 96:5310-5315.

Schroeder BC, Cheng T, Jan YN, and Jan LY (2008) Expression cloning of TMEM16A as a calcium-activated chloride channel subunit. Cell 134:1019-1029.

Schultz BD, DeRoos AD, Venglarik CJ, Singh AK, Frizzell RA, and Bridges RJ (1996) Glibenclamide blockade of CFTR chloride channels. Am J Physiol 271:L192-L200.

Schultz BD, Singh AK, Devor DC, and Bridges RJ (1999) Pharmacology of CFTR chloride channel activity. Physiol Rev 79 (Suppl):S109-S144.

Schultz BD, Takahashi A, Liu C, Frizzell RA, and Howard M (1997) FLAG epitope positioned in an external loop preserves normal biophysical properties of CFTR. Am J Physiol 273:C2080-C2089.

Schwiebert EM, Benos DJ, Egan ME, Stutts MJ, and Guggino WB (1999) CFTR is a conductance regulator as well as a chloride channel. Physiol Rev 79 (Suppl): S145-S166.

Schwiebert EM, Cid-Soto LP, Stafford D, Carter M, Blaisdell CJ, Zeitlin PL, Guggino WB, and Cutting GR (1998) Analysis of ClC-2 channels as an alternative pathway for chloride conduction in cystic fibrosis airway cells. Proc Natl Acad Sci USA 95: 3879-3884.

Schwiebert EM, Karlson KH, Friedman PA, Dietl P, Spielman WS, and Stanton BA (1992) Adenosine regulates a chloride channel via protein kinase $\mathrm{C}$ and a $\mathrm{G}$ protein in a rabbit cortical collecting duct cell line. J Clin Invest 89:834-841.

Scott-Ward TS, Li H, Schmidt A, Cai Z, and Sheppard DN (2004) Direct block of the cystic fibrosis transmembrane conductance regulator $\mathrm{Cl}(-)$ channel by niflumic acid. Mol Membr Biol 21:27-38.

Seifert S and Sontheimer H (2014) Bradykinin enhances invasion of malignant glioma into the brain parenchyma by inducing cells to undergo amoeboid migration. $J$ Physiol 592:5109-5127.

Senger R and Goldmann WH (1995) The influence of cations and ionic strength on actin polymerization in the presence/absence of alpha-actinin. Biochem Mol Biol Int 35:103-109.

Seo Y, Lee HK, Park J, Jeon DK, Jo S, Jo M, and Namkung W (2016) Ani9, a novel potent small-molecule ANO1 inhibitor with negligible effect on ANO2. PLoS One 11:e0155771.

Shen M, Wang L, Wang B, Wang T, Yang G, Shen L, Wang T, Guo X, Liu Y, Xia Y, et al. (2014) Activation of volume-sensitive outwardly rectifying chloride channel by ROS contributes to ER stress and cardiac contractile dysfunction: involvement of CHOP through Wnt. Cell Death Dis 5:e1528.

Shen MR, Wu SN, and Chou CY (1996) Volume-sensitive chloride channels in the primary culture cells of human cervical carcinoma. Biochim Biophys Acta 1315: 138-144.

Sheppard DN and Robinson KA (1997) Mechanism of glibenclamide inhibition of cystic fibrosis transmembrane conductance regulator $\mathrm{Cl}^{-}$channels expressed in a murine cell line. J Physiol 503:333-346.

Sheppard DN and Welsh MJ (1992) Effect of ATP-sensitive $\mathrm{K}^{+}$channel regulators on cystic fibrosis transmembrane conductance regulator chloride currents. J Gen Physiol 100:573-591.

Sheppard DN and Welsh MJ (1999) Structure and function of the CFTR chloride channel. Physiol Rev 79 (Suppl):S23-S45

Shimizu T, Iehara T, Sato K, Fujii T, Sakai H, and Okada Y (2013) TMEM16F is a component of a $\mathrm{Ca}^{2+}$-activated $\mathrm{Cl}^{-}$channel but not a volume-sensitive outwardly rectifying $\mathrm{Cl}^{-}$channel. Am J Physiol Cell Physiol 304:C748-C759.

Shimizu T, Lee EL, Ise T, and Okada Y (2008) Volume-sensitive Cl(-) channel as a regulator of acquired cisplatin resistance. Anticancer Res 28:75-83.
Shimizu T, Morishima S, and Okada Y (2000) $\mathrm{Ca}^{2+}$-sensing receptor-mediated regulation of volume-sensitive $\mathrm{Cl}^{-}$channels in human epithelial cells. J Physiol 528 $457-472$.

Shimizu T, Numata T, and Okada Y (2004) A role of reactive oxygen species in apoptotic activation of volume-sensitive Cl(-) channel. Proc Natl Acad Sci USA 101: $6770-6773$

Shuba YM, Prevarskaya N, Lemonnier L, Van Coppenolle F, Kostyuk PG, Mauroy B, and Skryma R (2000) Volume-regulated chloride conductance in the LNCaP human prostate cancer cell line. Am J Physiol Cell Physiol 279: C1144-C1154.

Siesjö BK (1988) Acidosis and ischemic brain damage. Neurochem Pathol 9:31-88.

Sirianant L, Ousingsawat J, Wanitchakool P, Schreiber R, and Kunzelmann K (2016) Cellular volume regulation by anoctamin $6: \mathrm{Ca}^{2+}$, phospholipase A2 and osmosensing. Pflugers Arch 468:335-349.

Sjöblom B, Salmazo A, and Djinović-Carugo K (2008) $\alpha$-actinin structure and regulation. Cell Mol Life Sci 65:2688-2701.

Smith RL, Clayton GH, Wilcox CL, Escudero KW, and Staley KJ (1995) Differential expression of an inwardly rectifying chloride conductance in rat brain neurons: a potential mechanism for cell-specific modulation of postsynaptic inhibition. $J$ Neurosci 15:4057-4067.

Snyder DS, Tradtrantip L, Yao C, Kurth MJ, and Verkman AS (2011) Potent, metabolically stable benzopyrimido-pyrrolo-oxazine-dione (BPO) CFTR inhibitors for polycystic kidney disease. J Med Chem 54:5468-5477.

Soejima M and Kokubun S (1988) Single anion-selective channel and its ion selectivity in the vascular smooth muscle cell. Pflugers Arch 411:304-311.

Sørensen BH, Dam CS, Stürup S, and Lambert IH (2016) Dual role of LRRC8Acontaining transporters on cisplatin resistance in human ovarian cancer cells. $J$ Inorg Biochem 160:287-295.

Sørensen BH, Thorsteinsdottir UA, and Lambert IH (2014) Acquired cisplatin resistance in human ovarian A2780 cancer cells correlates with shift in taurine homeostasis and ability to volume regulate. Am J Physiol Cell Physiol 307: C1071-C1080.

Stahl M, Stahl K, Brubacher MB, and Forrest JN Jr (2012) Divergent CFTR orthologs respond differently to the channel inhibitors CFTRinh-172, glibenclamide, and GlyH-101. Am J Physiol Cell Physiol 302:C67-C76.

Staley K, Smith R, Schaack J, Wilcox C, and Jentsch TJ (1996) Alteration of GABAA receptor function following gene transfer of the CLC-2 chloride channel. Neuron 17: $543-551$.

Stauber T (2015) The volume-regulated anion channel is formed by LRRC8 heteromers - molecular identification and roles in membrane transport and physiology. Biol Chem 396:975-990.

Stephan AB, Shum EY, Hirsh S, Cygnar KD, Reisert J, and Zhao H (2009) ANO2 is the cilial calcium-activated chloride channel that may mediate olfactory amplification. Proc Natl Acad Sci USA 106:11776-11781.

Stöhr H, Heisig JB, Benz PM, Schöberl S, Milenkovic VM, Strauss O, Aartsen WM, Wijnholds J, Weber BH, and Schulz HL (2009) TMEM16B, a novel protein with calcium-dependent chloride channel activity, associates with a presynaptic protein complex in photoreceptor terminals. J Neurosci 29:6809-6818.

Stott JB, deCourcey F, Ennis M, and Zholos AV (2014) Functional and pharmacological characterization of volume-regulated anion channels in human normal and cystic fibrosis bronchial and nasal epithelial cells. Eur J Pharmacol 740:183-191.

Strange K, Emma F, and Jackson PS (1996) Cellular and molecular physiology of volume-sensitive anion channels. Am J Physiol 270:C711-C730.

Stroffekova K, Kupert EY, Malinowska DH, and Cuppoletti J (1998) Identification of the $\mathrm{pH}$ sensor and activation by chemical modification of the $\mathrm{ClC}-2 \mathrm{G} \mathrm{Cl}^{-}$channel. Am J Physiol 275:C1113-C1123.

Strotmann R, Harteneck C, Nunnenmacher K, Schultz G, and Plant TD (2000) OTRPC4, a nonselective cation channel that confers sensitivity to extracellular osmolarity. Nat Cell Biol 2:695-702.

Sun H, Tsunenari T, Yau KW, and Nathans J (2002) The vitelliform macular dystrophy protein defines a new family of chloride channels. Proc Natl Acad Sci USA 99:4008-4013.

Sun XP, Supplisson S, and Mayer E (1993) Chloride channels in myocytes from rabbit colon are regulated by a pertussis toxin-sensitive G protein. Am J Physiol 264 G774-G785.

Sun XP, Supplisson S, Torres R, Sachs G, and Mayer E (1992) Characterization of large-conductance chloride channels in rabbit colonic smooth muscle. J Physiol 448:355-382

Suzuki J, Umeda M, Sims PJ, and Nagata S (2010) Calcium-dependent phospholipid scrambling by TMEM16F. Nature 468:834-838.

Suzuki T, Suzuki J, and Nagata S (2014) Functional swapping between transmembrane proteins TMEM16A and TMEM16F. J Biol Chem 289:7438-7447.

Syeda R, Qiu Z, Dubin AE, Murthy SE, Florendo MN, Mason DE, Mathur J, Cahalan SM, Peters EC, Montal M, et al. (2016) LRRC8 proteins form volume-regulated anion channels that sense ionic strength. Cell 164:499-511.

Ternovsky VI, Okada Y, and Sabirov RZ (2004) Sizing the pore of the volumesensitive anion channel by differential polymer partitioning. FEBS Lett 576: 433-436.

Thiemann A, Gründer S, Pusch M, and Jentsch TJ (1992) A chloride channel widely expressed in epithelial and non-epithelial cells. Nature 356:57-60.

Thompson CH, Fields DM, Olivetti PR, Fuller MD, Zhang ZR, Kubanek J, and McCarty NA (2005) Inhibition of ClC-2 chloride channels by a peptide component or components of scorpion venom. J Membr Biol 208:65-76.

Thompson CH, Olivetti PR, Fuller MD, Freeman CS, McMaster D, French RJ, Pohl J, Kubanek J, and McCarty NA (2009) Isolation and characterization of a high affinity peptide inhibitor of ClC-2 chloride channels. J Biol Chem 284: 26051-26062

Tokimasa T and North RA (1996) Effects of barium, lanthanum and gadolinium on endogenous chloride and potassium currents in Xenopus oocytes. J Physiol 496 $677-686$. 
Tominaga M, Horie M, Sasayama S, and Okada Y (1995a) Glibenclamide, an ATPsensitive $\mathrm{K}^{+}$channel blocker, inhibits cardiac cAMP-activated $\mathrm{Cl}^{-}$conductance. Circ Res 77:417-423.

Tominaga M, Tominaga T, Miwa A, and Okada Y (1995b) Volume-sensitive chloride channel activity does not depend on endogenous P-glycoprotein. J Biol Chem 270: 27887-27893.

Toychiev AH, Sabirov RZ, Takahashi N, Ando-Akatsuka Y, Liu H, Shintani T, Noda M, and Okada Y (2009) Activation of maxi-anion channel by protein tyrosine dephosphorylation. Am J Physiol Cell Physiol 297:C990-C1000.

Tradtrantip L, Sonawane ND, Namkung W, and Verkman AS (2009) Nanomolar potency pyrimido-pyrrolo-quinoxalinedione CFTR inhibitor reduces cyst size in a polycystic kidney disease model. J Med Chem 52:6447-6455.

Tsumura T, Oiki S, Ueda S, Okuma M, and Okada Y (1996) Sensitivity of volumesensitive $\mathrm{Cl}^{-}$conductance in human epithelial cells to extracellular nucleotides. $\mathrm{Am}$ J Physiol 271:C1872-C1878.

Tsunenari T, Nathans J, and Yau KW (2006) $\mathrm{Ca}^{2+}$-activated $\mathrm{Cl}^{-}$current from human bestrophin-4 in excised membrane patches. J Gen Physiol 127: $749-754$

Ullrich F, Reincke SM, Voss FK, Stauber T, and Jentsch TJ (2016) Inactivation and anion selectivity of volume-regulated anion channels (VRACs) depend on C-terminal residues of the first extracellular loop. $J$ Biol Chem 291 17040-17048

Valverde MA, O'Brien JA, Sepúlveda FV, Ratcliff R, Evans MJ, and Colledge WH (1993) Inactivation of the murine cftr gene abolishes cAMP-mediated but not $\mathrm{Ca}(2+)$-mediated secretagogue-induced volume decrease in small-intestinal crypts. Pflugers Arch 425:434-438.

Valverde MA, O'Brien JA, Sepúlveda FV, Ratcliff RA, Evans MJ, and Colledge WH (1995) Impaired cell volume regulation in intestinal crypt epithelia of cystic fibrosis mice. Proc Natl Acad Sci USA 92:9038-9041.

Varela D, Simon F, Olivero P, Armisén R, Leiva-Salcedo E, Jørgensen F, Sala F, and Stutzin A (2007) Activation of $\mathrm{H}_{2} \mathrm{O}_{2}$-induced VSOR Cl${ }^{-}$currents in HTC cells require phospholipase Cgamma1 phosphorylation and $\mathrm{Ca}^{2+}$ mobilisation. Cell Physiol Biochem 20:773-780.

Varela D, Simon F, Riveros A, Jørgensen F, and Stutzin A (2004) NAD(P)H oxidasederived $\mathrm{H}(2) \mathrm{O}(2)$ signals chloride channel activation in cell volume regulation and cell proliferation. J Biol Chem 279:13301-13304.

Vázquez E, Nobles M, and Valverde MA (2001) Defective regulatory volume decrease in human cystic fibrosis tracheal cells because of altered regulation of intermediate conductance $\mathrm{Ca}^{2+}$-dependent potassium channels. Proc Natl Acad Sci USA 98: 5329-5334.

Venglarik CJ, Schultz BD, DeRoos AD, Singh AK, and Bridges RJ (1996) Tolbutamide causes open channel blockade of cystic fibrosis transmembrane conductance regulator $\mathrm{Cl}^{-}$channels. Biophys $J$ 70:2696-2703.

Vennekens R, Trouet D, Vankeerberghen A, Voets T, Cuppens H, Eggermont J, Cassiman JJ, Droogmans G, and Nilius B (1999) Inhibition of volume-regulated anion channels by expression of the cystic fibrosis transmembrane conductance regulator. $J$ Physiol 515:75-85.

Voets T, Buyse G, Tytgat J, Droogmans G, Eggermont J, and Nilius B (1996) The chloride current induced by expression of the protein $\mathrm{pI}_{\mathrm{Cln}}$ in Xenopus oocytes differs from the endogenous volume-sensitive chloride current. $J$ Physiol 495 441-447.

Voets T, Droogmans G, Raskin G, Eggermont J, and Nilius B (1999) Reduced intracellular ionic strength as the initial trigger for activation of endothelial volume-regulated anion channels. Proc Natl Acad Sci USA 96 5298-5303.

Voets T, Wei L, De Smet P, Van Driessche W, Eggermont J, Droogmans G, and Nilius B (1997) Downregulation of volume-activated $\mathrm{Cl}^{-}$currents during muscle differentiation. Am J Physiol 272:C667-C674.

Voss FK, Ullrich F, Münch J, Lazarow K, Lutter D, Mah N, Andrade-Navarro MA, von Kries JP, Stauber T, and Jentsch TJ (2014) Identification of LRRC8 heteromers as an essential component of the volume-regulated anion channel VRAC. Science 344:634-638.

Wang F, Zeltwanger S, Yang IC, Nairn AC, and Hwang TC (1998) Actions of genistein on cystic fibrosis transmembrane conductance regulator channel gating. Evidence for two binding sites with opposite effects. J Gen Physiol 111:477-490.

Wang HY, Shimizu T, Numata T, and Okada Y (2007) Role of acid-sensitive outwardly rectifying anion channels in acidosis-induced cell death in human epithelial cells. Pflugers Arch 454:223-233.

Wang L, Ma W, Zhu L, Ye D, Li Y, Liu S, Li H, Zuo W, Li B, Ye W, et al. (2012) ClC-3 is a candidate of the channel proteins mediating acid-activated chloride currents in nasopharyngeal carcinoma cells. Am J Physiol Cell Physiol 303:C14-C23.

Wang L, Shen M, Guo X, Wang B, Xia Y, Wang N, Zhang Q, Jia L, and Wang X (2017a) Volume-sensitive outwardly rectifying chloride channel blockers protect against high glucose-induced apoptosis of cardiomyocytes via autophagy activation. Sci Rep 7:44265.

Wang Q, Hogg RC, and Large WA (1992) Properties of spontaneous inward currents recorded in smooth muscle cells isolated from the rabbit portal vein. J Physiol $\mathbf{4 5 1}$ 525-537

Wang R, Lu Y, Gunasekar S, Zhang Y, Benson CJ, Chapleau MW, Sah R, and Abboud FM (2017b) The volume-regulated anion channel (LRRC8) in nodose neurons is sensitive to acidic pH. JCI Insight 2:e90632.

Wang T, Birsoy K, Hughes NW, Krupczak KM, Post Y, Wei JJ, Lander ES, and Sabatini DM (2015) Identification and characterization of essential genes in the human genome. Science 350:1096-1101.

Wang X, Bompadre SG, Li M, and Hwang TC (2009) Mutations at the signature sequence of CFTR create a Cd(2+)-gated chloride channel. J Gen Physiol 133 69-77.

Wang X, Takahashi N, Uramoto H, and Okada Y (2005) Chloride channel inhibition prevents ROS-dependent apoptosis induced by ischemia-reperfusion in mouse cardiomyocytes. Cell Physiol Biochem 16:147-154
Wang Z, Mitsuiye T, Rees SA, and Noma A (1997) Regulatory volume decrease of cardiac myocytes induced by beta-adrenergic activation of the $\mathrm{Cl}^{-}$channel in guinea pig. J Gen Physiol 110:73-82.

Wangemann P, Wittner M, Di Stefano A, Englert HC, Lang HJ, Schlatter E, and Greger R (1986) Cl(-)-channel blockers in the thick ascending limb of the loop of Henle. Structure activity relationship. Pflugers Arch 407 (Suppl 2): S128-S141.

Waniishi Y, Inoue R, Morita H, Teramoto N, Abe K, and Ito Y (1998) Cyclic GMPdependent but G-kinase-independent inhibition of $\mathrm{Ca}^{2+}$-dependent $\mathrm{Cl}^{-}$currents by NO donors in cat tracheal smooth muscle. J Physiol 511:719-731.

Wei L, Vankeerberghen A, Cuppens H, Cassiman JJ, Droogmans G, and Nilius B (2001) The C-terminal part of the R-domain, but not the PDZ binding motif, of CFTR is involved in interaction with $\mathrm{Ca}(2+)$-activated $\mathrm{Cl}^{-}$channels. Pflugers Arch 442:280-285.

Weinreich $\mathrm{F}$ and Jentsch TJ (2001) Pores formed by single subunits in mixed dimers of different CLC chloride channels. $J$ Biol Chem 276:2347-2353.

White MM and Aylwin M (1990) Niflumic and flufenamic acids are potent reversible blockers of $\mathrm{Ca} 2(+)$-activated $\mathrm{Cl}^{-}$channels in Xenopus oocytes. Mol Pharmacol 37: 720-724.

Winder CV, Wax J, Serrano B, Jones EM, and McPhee ML (1963) Anti-inflammatory and antipyretic properties of $\mathrm{N}$-(alpha,alpha,alpha-trifluoro-m-tolyl) anthranilic acid (CI-440; flufenamic acid). Arthritis Rheum 6:36-47.

Winpenny JP, Harris A, Hollingsworth MA, Argent BE, and Gray MA (1998) Calcium-activated chloride conductance in a pancreatic adenocarcinoma cell line of ductal origin (HPAF) and in freshly isolated human pancreatic duct cells. Pflugers Arch 435:796-803.

Winpenny JP, Mathews CJ, Verdon B, Wardle CJ, Chambers JA, Harris A, Argent BE, and Gray MA (1996) Volume-sensitive chloride currents in primary cultures of human fetal vas deferens epithelial cells. Pflugers Arch $\mathbf{4 3 2}$ $644-654$

Wladkowski SL, Lin W, McPheeters M, Kinnamon SC, and Mierson S (1998) A basolateral chloride conductance in rat lingual epithelium. J Membr Biol 164: 91-101.

Wondergem R, Gong W, Monen SH, Dooley SN, Gonce JL, Conner TD, Houser M, Ecay TW, and Ferslew KE (2001) Blocking swelling-activated chloride current inhibits mouse liver cell proliferation. J Physiol 532:661-672.

Worrell RT, Butt AG, Cliff WH, and Frizzell RA (1989) A volume-sensitive chloride conductance in human colonic cell line T84. Am J Physiol 256:C1111-C1119.

Wu G and Hamill OP (1992) NPPB block of $\mathrm{Ca}(++)$-activated Cl- currents in Xenopus oocytes. Pflugers Arch 420:227-229.

Xia Y, Liu Y, Xia T, Li X, Huo C, Jia X, Wang L, Xu R, Wang N, Zhang M, et al. (2016) Activation of volume-sensitive $\mathrm{Cl}^{-}$channel mediates autophagy-related cell death in myocardial ischaemia/reperfusion injury. Oncotarget 7:39345-39362.

Xiao Q, Hartzell HC, and Yu K (2010) Bestrophins and retinopathies. Pflugers Arch 460:559-569.

Xiao Q, Yu K, Cui YY, and Hartzell HC (2009) Dysregulation of human bestrophin-1 by ceramide-induced dephosphorylation. J Physiol 587:4379-4391.

Xie C, Cao X, Chen X, Wang D, Zhang WK, Sun Y, Hu W, Zhou Z, Wang Y, and Huang $P$ (2016) Mechanosensitivity of wild-type and G551D cystic fibrosis transmembrane conductance regulator (CFTR) controls regulatory volume decrease in simple epithelia. FASEB $J$ 30:1579-1589.

Xiong H, Li C, Garami E, Wang Y, Ramjeesingh M, Galley K, and Bear CE (1999) ClC-2 activation modulates regulatory volume decrease. J Membr Biol 167 $215-221$.

Xiong ZG, Zhu XM, Chu XP, Minami M, Hey J, Wei WL, MacDonald JF, Wemmie JA Price MP, Welsh MJ, et al. (2004) Neuroprotection in ischemia: blocking calciumpermeable acid-sensing ion channels. Cell 118:687-698.

Yamada T and Strange K (2018) Intracellular and extracellular loops of LRRC8 are essential for volume-regulated anion channel function. J Gen Physiol 150 $1003-1015$

Yamamoto S and Ehara T (2006) Acidic extracellular pH-activated outwardly rectifying chloride current in mammalian cardiac myocytes. Am J Physiol Heart Circ Physiol 290:H1905-H1914

Yamamoto S, Ehara T, and Shioya T (2001) Changes in cell volume induced by activation of the cyclic amp-dependent chloride channel in guinea-pig cardiac myocytes. Jpn J Physiol 51:31-41.

Yamamura H, Nishimura K, Hagihara Y, Suzuki Y, and Imaizumi Y (2018) TMEM16A and TMEM16B channel proteins generate $\mathrm{Ca}^{2+}$-activated $\mathrm{Cl}^{-}$current and regulate melatonin secretion in rat pineal glands. J Biol Chem $\mathbf{2 9 3}$ 995-1006.

Yamazaki J and Hume JR (1997) Inhibitory effects of glibenclamide on cystic fibrosis transmembrane regulator, swelling-activated, and $\mathrm{Ca}(2+)$-activated $\mathrm{Cl}^{-}$channels in mammalian cardiac myocytes. Circ Res 81:101-109.

Yang H, Kim A, David T, Palmer D, Jin T, Tien J, Huang F, Cheng T, Coughlin SR, Jan YN, et al. (2012) TMEM16F forms a $\mathrm{Ca}^{2+}$-activated cation channel required for lipid scrambling in platelets during blood coagulation. Cell 151:111-122.

Yang X, Zhu L, Lin J, Liu S, Luo H, Mao J, Nie S, Chen L, and Wang L (2015) Cisplatin activates volume-sensitive like chloride channels via purinergic receptor pathways in nasopharyngeal carcinoma cells. $J$ Membr Biol 248:19-29.

Yang XC and Sachs F (1989) Block of stretch-activated ion channels in Xenopus oocytes by gadolinium and calcium ions. Science 243:1068-1071.

Yang YD, Cho H, Koo JY, Tak MH, Cho Y, Shim WS, Park SP, Lee J, Lee B, Kim BM, et al. (2008) TMEM16A confers receptor-activated calcium-dependent chloride conductance. Nature 455:1210-1215.

Ye ZC, Oberheim N, Kettenmann H, and Ransom BR (2009) Pharmacological "crossinhibition" of connexin hemichannels and swelling activated anion channels. Glia 57:258-269.

Yermolaieva O, Leonard AS, Schnizler MK, Abboud FM, and Welsh MJ (2004) Extracellular acidosis increases neuronal cell calcium by activating acid-sensing ion channel 1a. Proc Natl Acad Sci USA 101:6752-6757. 
Yu K, Whitlock JM, Lee K, Ortlund EA, Cui YY, and Hartzell HC (2015) Identification of a lipid scrambling domain in ANO6/TMEM16F. eLife 4:e6901.

Yu K, Xiao Q, Cui G, Lee A, and Hartzell HC (2008) The best disease-linked $\mathrm{Cl}^{-}$ channel hBest1 regulates Ca V 1 (L-type) $\mathrm{Ca}^{2+}$ channels via $s r c$-homology-binding domains. J Neurosci 28:5660-5670.

Zegarra-Moran O and Galietta LJ (2017) CFTR pharmacology. Cell Mol Life Sci 74 117-128.

Zhang H, Cao HJ, Kimelberg HK, and Zhou M (2011) Volume regulated anion channel currents of rat hippocampal neurons and their contribution to oxygen-andglucose deprivation induced neuronal death. PLoS One 6:e16803.

Zhang JJ, Jacob TJ, Valverde MA, Hardy SP, Mintenig GM, Sepúlveda FV, Gill DR, Hyde SC, Trezise AE, and Higgins CF (1994) Tamoxifen blocks chloride channels. A possible mechanism for cataract formation. J Clin Invest 94:1690-1697.

Zhang WK, Wang D, Duan Y, Loy MM, Chan HC, and Huang P (2010a) Mechanosensitive gating of CFTR. Nat Cell Biol 12:507-512.

Zhang Y, Stanton JB, Wu J, Yu K, Hartzell HC, Peachey NS, Marmorstein LY, and Marmorstein $\mathrm{AD}(2010 \mathrm{~b})$ Suppression of $\mathrm{Ca}^{2+}$ signaling in a mouse model of Best disease. Hum Mol Genet 19:1108-1118.

Zhang Z, He JW, Fu WZ, Zhang CQ, and Zhang ZL (2014) Two novel mutations in the SLCO2A1 gene in a Chinese patient with primary hypertrophic osteoarthropathy. Gene 534:421-423.

Zhang Z, Liu F, and Chen J (2017) Conformational changes of CFTR upon phosphorylation and ATP binding. Cell 170:483-491.e8.
Zhang Z, Xia W, He J, Zhang Z, Ke Y, Yue H, Wang C, Zhang H, Gu J, Hu W, et al. (2012) Exome sequencing identifies SLCO2A1 mutations as a cause of primary hypertrophic osteoarthropathy. Am J Hum Genet 90:125-132.

Zhou JJ, Li MS, Qi J, and Linsdell P (2010) Regulation of conductance by the number of fixed positive charges in the intracellular vestibule of the CFTR chloride channel pore. J Gen Physiol 135:229-245.

Zhou JJ and Linsdell P (2007) Molecular mechanism of arachidonic acid inhibition of the CFTR chloride channel. Eur J Pharmacol 563:88-91.

Zhou P, Polovitskaya MM, and Jentsch TJ (2018) LRRC8 N termini influence pore properties and gating of volume-regulated anion channels (VRACs). J Biol Chem 293:13440-13451.

Zhou SS, Hazama A, and Okada Y (1998) Tyrosine kinase-independent extracellular action of genistein on the CFTR $\mathrm{Cl}^{-}$channel in guinea pig ventricular myocytes and CFTR-transfected mouse fibroblasts. Jpn J Physiol 48:389-396.

Zhou SS, Takai A, Tominaga M, and Okada Y (1997) Phosphatase-mediated enhancement of cardiac cAMP-activated Cl-conductance by a $\mathrm{Cl}^{-}$channel blocker, anthracene-9-carboxylate. Circ Res 81:219-228.

Zitt C, Zobel A, Obukhov AG, Harteneck C, Kalkbrenner F, Lückhoff A, and Schultz G (1996) Cloning and functional expression of a human $\mathrm{Ca}^{2}$ ${ }^{+}$-permeable cation channel activated by calcium store depletion. Neuron 16: 1189-1196.

Zúñiga L, Niemeyer MI, Varela D, Catalán M, Cid LP, and Sepúlveda FV (2004) The voltage-dependent ClC-2 chloride channel has a dual gating mechanism. J Physiol 555:671-682. 